\title{
Smash Products for Secondary Homotopy Groups
}

\author{
Hans-Joachim Baues • Fernando Muro
}

Received: 13 June 2006 / Accepted: 20 February 2007 / Published online: 2 October 2007

(C) Springer Science + Business Media B.V. 2007

\begin{abstract}
We construct a smash product operation on secondary homotopy groups yielding the structure of a lax symmetric monoidal functor. Applications on cup-one products, Toda brackets and Whitehead products are considered.
\end{abstract}

Keywords Secondary homotopy group • Square group • Crossed module • Smash product $\cdot$ Lax symmetric monoidal functor - Whitehead product • Cup-one product . Toda bracket

Mathematics Subject Classifications (2000) 55Q15 · 55Q35 · 55S45

\section{Introduction}

The classical homotopy groups $\pi_{n} X, n \geq 0$, of a pointed space $X$ give rise to a graded abelian group $\Pi_{*} X$ obtained by additivization in low dimensions. In particular $\Pi_{n} X=\pi_{n} X$ for $n \geq 2, \Pi_{1} X=\left(\pi_{1} X\right)_{a b}$ is the abelianized fundamental group, and $\Pi_{0} X=\mathbb{Z}\left[\pi_{0} X\right]$ is the free abelian group on the pointed set of path components of $X$. The smash product on homotopy groups induces a natural homomorphism of graded abelian groups

$$
\Pi_{*} X \otimes \Pi_{*} Y \stackrel{\wedge}{\longrightarrow} \Pi_{*}(X \wedge Y)
$$

\footnotetext{
The second author was partially supported by the Spanish Ministry of Education and Science under MEC-FEDER grants MTM2004-01865 and MTM2004-03629, the postdoctoral fellowship EX2004-0616, and a Juan de la Cierva research contract.
}

H.-J. Baues

Max-Planck-Institut für Mathematik, Vivatsgasse 7, 53111 Bonn, Germany

e-mail: baues@mpim-bonn.mpg.de

F. Muro $(\varangle)$

Departament d'Àlgebra i Geometria, Universitat de Barcelona, Gran Via de les Corts Catalanes, 585, 08007 Barcelona, Spain e-mail: fmuro@ub.edu 
which carries $f \otimes g$, with $f: S^{n} \rightarrow X$ and $g: S^{m} \rightarrow Y$, to $f \wedge g: S^{n+m} \rightarrow X \wedge Y$. This shows that $\Pi_{*}$ is a lax symmetric monoidal functor from pointed spaces to graded abelian groups.

The smash product (1) can be used for example to define the Whitehead product on homotopy groups, compare Section 3.4.

The purpose of this paper is to generalize these properties of primary homotopy groups on the level of secondary homotopy theory.

Secondary homotopy operations like Toda brackets [22] or cup-one products $[6,18]$ are defined by pasting tracks, where tracks are homotopy classes of homotopies. Since secondary homotopy operations play a crucial role in homotopy theory it is of importance to develop the algebraic theory of tracks. We do this by introducing secondary homotopy groups of a pointed space $X$

$$
\Pi_{n, *} X=\left(\Pi_{n, 1} X \stackrel{\partial}{\rightarrow} \Pi_{n, 0} X\right)
$$

which have the structure of a quadratic pair module, see Sections 2.1 and 3.1. Here $\partial$ is a group homomorphism with cokernel $\Pi_{n} X$ for $n \geq 0$ and kernel $\Pi_{n+1} X$ for $n \geq 3$.

We define $\Pi_{n, *} X$ for $n \geq 2$ directly in terms of maps $S^{n} \rightarrow X$ and tracks from such maps to the trivial map. For $n \geq 0$ the functor $\Pi_{n, *}$ is an additive version of the functor $\pi_{n, *}$ studied in [9].

We introduce and study the smash product morphism for additive secondary homotopy groups

$$
\Pi_{*, *} X \odot \Pi_{*, *} Y \stackrel{\wedge}{\longrightarrow} \Pi_{*, *}(X \wedge Y) .
$$

Here one needs the symmetric monoidal structure $\odot$ of the category of quadratic pair modules qpm, which is based on the symmetric monoidal structure on the category of square groups constructed in [7]. The smash product morphism (2) is compatible with the associativity isomorphisms, but it is not directly compatible with the commutativity isomorphisms.

In order to deal with commutativity we need the action of the symmetric track group $\operatorname{Sym}_{\square}(n)$ on $\Pi_{n, *} X$ in [10]. We show that $\wedge$ in Eq. 2 is equivariant with respect to this action, and is commutative up to the action of a shuffle permutation. This

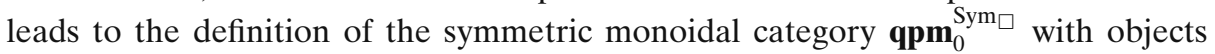
given by symmetric sequences of quadratic pair modules with extra structure. Then the morphism (2) induces a morphism in $\mathbf{q p m}_{0}^{\mathrm{Sym}} \square$ for which the associativity and commutativity isomorphisms are compatible with the symmetric monoidal structure of $\mathbf{q p m}_{0}^{\mathrm{Sym}} \square$. Therefore $\Pi_{*, *}$ considered as a functor to the category $\mathbf{q p m}_{0}^{\mathrm{Sym}} \square$ is, in fact, a lax symmetric monoidal functor.

The smash product (2) is used to define the Whitehead product on secondary homotopy groups, compare Section 3.4.

As an illustrating application of the results in this paper we prove a formula of Barratt-Jones-Mahowald on unstable cup-one products, see Section 3.5. This formula was stated in [6], but a proof did not appear in the literature. A further application yields a formula for a triple Toda bracket which generalizes a well-known formula in [22], see Section 3.6.

In a sequel of this paper we generalize the theory of secondary homotopy groups to symmetric spectra [8]. There we show that the smash product operation defined in this paper endows the secondary homotopy groups $\pi_{*, *} R$ of a fibrant connective 
ring spectrum $R$ with a graded algebra structure in the category of quadratic pair modules. The graded algebra $\pi_{*, *} R$ determines all Toda brackets in $\pi_{*} R$, which can be regarded as Massey products in $\pi_{*, *} R$. Moreover, $\pi_{*, *} R$ determines the universal matrix Toda bracket in the category of finitely generated free $R$-modules. If $R$ is in addition an $E_{\infty}$-ring spectrum then $\pi_{*, *} R$ is a commutative algebra up to coherent homotopies in $\mathbf{q p m}_{0}^{\text {Sym }} \square$ which encodes not only Toda brackets, but also cup-one products in a purely algebraic way.

The paper consists of three parts. The first part is concerned with the algebra needed for the statements of the main theorems. In Section 3 we present our main results and we give applications. Section 4 contains the construction of the smash product operation for additive secondary homotopy groups. There we prove all the properties which imply our main results.

\section{Quadratic Pair Modules and Their Tensor Product}

In this part we describe the algebraic concepts needed for the structure of secondary homotopy groups. We introduce the category of quadratic pair modules and we show that this category is symmetric monoidal. The tensor product of quadratic pair modules is related to the exterior cup-products in the category Top*.

\subsection{Square Groups and Quadratic Pair Modules}

We first recall the notion of square group, see [13] and [7].

Definition 2.1.1 A square group $X$ is a diagram

$$
X=\left(X_{e} \underset{H}{\stackrel{P}{\leftrightarrows}} X_{e e}\right)
$$

where $X_{e}$ is a group with an additively written group law, $X_{e e}$ is an abelian group, $P$ is a homomorphism, $H$ is a quadratic map, i.e. a function such that the cross effect

$$
(a \mid b)_{H}=H(a+b)-H(b)-H(a)
$$

is linear in $a, b \in X_{e}$, and the following relations are satisfied, $x, y \in X_{e e}$,

(1) $(P x \mid b)_{H}=0,(a \mid P y)=0$,

(2) $P(a \mid b)_{H}=[a, b]$,

(3) $P H P(x)=P(x)+P(x)$.

Here $[a, b]=-a-b+a+b$ is the commutator bracket. The cross effect induces a homomorphism

$$
(-\mid-)_{H}: \otimes^{2} \text { Coker } P \longrightarrow X_{e e} .
$$

Here $\otimes^{2} A=A \otimes A$ is the tensor square of an abelian group $A$. If Eq. 3 is an isomorphism we will say that the square group $X$ is good. The function

$$
T=H P-1: X_{e e} \longrightarrow X_{e e}
$$


is an involution, i.e. a homomorphism with $T^{2}=1$. Moreover,

$$
\Delta: X_{e} \longrightarrow X_{e e}: x \mapsto(x \mid x)_{H}-H(x)+T H(x)
$$

is a homomorphism which satisfies $T \Delta=-\Delta$.

A morphism of square groups $f: X \rightarrow Y$ is given by homomorphisms $f_{e}: X_{e} \rightarrow$ $Y_{e}, f_{e e}: X_{e e} \rightarrow Y_{e e}$, commuting with $P$ and $H$.

As an example of square group we can consider

$$
\mathbb{Z}_{n i l}=(\underset{\mathbb{Z} \underset{H}{\stackrel{P}{\leftrightarrows}} \mathbb{Z}}{)})
$$

with $P=0$ and $H(n)=\left(\begin{array}{l}n \\ 2\end{array}\right)=\frac{n(n-1)}{2}$. This is the unit object of the symmetric monoidal structure defined in the next section.

We refer the reader to [7] where the quadratic algebra of square groups is developed. We need square groups for the definition of quadratic pair modules as follows.

Definition 2.1.2 A quadratic pair module $C$ is a morphism $\partial: C_{(1)} \rightarrow C_{(0)}$ between square groups

$$
\begin{aligned}
& C_{(0)}=\left(C_{0} \underset{H}{\stackrel{P_{0}}{\leftrightarrows}} C_{e e}\right), \\
& C_{(1)}=\left(C_{1} \underset{H_{1}}{\stackrel{P}{\leftrightarrows}} C_{e e}\right),
\end{aligned}
$$

such that $\partial_{e e}=1: C_{e e} \rightarrow C_{e e}$ is the identity homomorphism. In particular $C$ is completely determined by the diagram

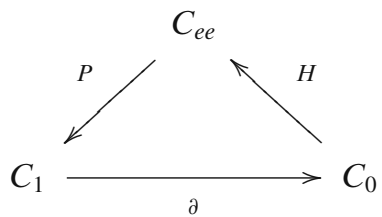

where $\partial=\partial_{e}, H_{1}=H \partial$ and $P_{0}=\partial P$.

Morphisms of quadratic pair modules $f: C \rightarrow D$ are therefore given by group homomorphisms $f_{0}: C_{0} \rightarrow D_{0}, f_{1}: C_{1} \rightarrow D_{1}, f_{e e}: C_{e e} \rightarrow D_{e e}$, commuting with $H, P$ and $\partial$ in Eq. 4. They form a category denoted by $\mathbf{q p m}$.

The homology of a quadratic pair module $C$ is given by the abelian groups

$$
\begin{aligned}
& h_{0} C=C_{0} / \partial\left(C_{1}\right), \\
& h_{1} C=\operatorname{Ker}\left[\partial: C_{1} \rightarrow C_{0}\right] .
\end{aligned}
$$

A morphism in $\mathbf{q p m}$ is a weak equivalence if it induces isomorphisms in homology. 空 Springer 
A quadratic pair module $C$ is 0 -good if the square group $C_{(0)}$ is good. The full subcategory of 0-good quadratic pair modules will be denoted by $\mathbf{q p m}_{0} \subset \mathbf{q p m}$.

Remark 2.1.3 The category squad of stable quadratic modules is described in [2, IV.C] and [9]. Quadratic modules in general are discussed in [2] and [17], they are special 2-crossed modules in the sense of [15]. There is a faithful forgetful functor qpm $\rightarrow$ squad sending $C$ to the stable quadratic module

$$
\otimes^{2}\left(C_{0}\right)_{a b} \stackrel{P(-\mid-)_{H}}{\longrightarrow} C_{1} \stackrel{\partial}{\longrightarrow} C_{0} .
$$

Here $G_{a b}$ denotes the abelianization of a group $G$. Such a stable quadratic module gives rise to a crossed module $\partial: C_{1} \rightarrow C_{0}$ where $C_{0}$ acts on the right of $C_{1}$ by the formula, $x \in C_{1}, y \in C_{0}$,

$$
x^{y}=x+P(\partial(x) \mid y)_{H}
$$

so that we also get a forgetful functor $\mathbf{q p m} \rightarrow$ cross to the track category of crossed modules. Tracks in qpm (i.e. invertible 2-morphisms) will be considered in Section 2.5 below by using this forgetful functor.

\subsection{The Tensor Product of Square Groups}

We now recall the notion of tensor product of square groups which is essential for this paper. This tensor product, first defined in [7], originates from properties of the exterior cup-products in the next section and in Section 4.

Definition 2.2.1 The tensor product $X \odot Y$ of square groups $X, Y$ is defined as follows. The group $(X \odot Y)_{e}$ is generated by the symbols $x \odot y, x \odot y, a \bar{\otimes} b$ for $x \in X_{e}$, $y \in Y_{e}, a \in X_{e e}$ and $b \in Y_{e e}$, subject to the following relations.

(1) The symbol $a \bar{\otimes} b$ is bilinear and central,

(2) The symbol $x @ y$ is right linear, $x @\left(y_{1}+y_{2}\right)=x @ y_{1}+x @ y_{2}$,

(3) The symbol $x \odot y$ is left linear, $\left(x_{1}+x_{2}\right) \odot y=x_{1} \odot y+\bar{x}_{2} \odot y$,

(4) $P(a) \odot y=a \bar{\otimes} \Delta(y)$,

(5) $x \odot \bar{P}(b)=\Delta(x) \bar{\otimes} b$,

(6) $T(a) \bar{\otimes} T(b)=-a \bar{\otimes} b$,

(7) $x @ y-x \odot y=H(x) \bar{\otimes} T H(y)$.

The abelian group $(X \odot Y)_{e e}$ is defined as the tensor product $X_{e e} \otimes Y_{e e}$. The homomorphism

$$
P:(X \odot Y)_{e e} \longrightarrow(X \odot Y)_{e}
$$

is $P(a \otimes b)=a \bar{\otimes} b$, and

$$
H:(X \odot Y)_{e} \longrightarrow(X \odot Y)_{e e}
$$


is the unique quadratic map satisfying

$$
\begin{aligned}
& H(x \odot y)=(x \mid x)_{H} \otimes H(y)+H(x) \otimes \Delta(y), \\
& H(x \odot y)=\Delta(x) \otimes H(y)+H(x) \otimes(y \mid y)_{H}, \\
& H(a \bar{\otimes} b)=a \otimes b-T(a) \otimes T(b), \\
& (a \bar{\otimes} b \mid-)_{H}=(-\mid a \bar{\otimes} b)_{H}=0, \\
& (a \odot b \mid c \odot d)_{H}=(a @ b \mid c \odot d)_{H} \\
& =(a \odot b \mid c \odot d)_{H} \\
& =(a \odot b \mid c \odot d)_{H} \\
& =(a \mid c)_{H} \otimes(b \mid d)_{H} .
\end{aligned}
$$

Relation (7) above shows that $(X \odot Y)_{e}$ is actually generated just by $x \odot y$ and $a \bar{\otimes} b$. A complete list of relations for these generators is given by (1), (2), (4) and (6) above together with

(8) $\left(x_{1}+x_{2}\right) @ y=x_{1} @ y+x_{2} @ y+\left(x_{2} \mid x_{1}\right)_{H} \bar{\otimes} H(y)$,

(9) $x @ P(b)=(x \mid x)_{H} \bar{\otimes} b$.

Similarly $(X \odot Y)_{e}$ is also generated by just $x \odot y$ and $a \bar{\otimes} b$ with relations (1), (3), (5) and (6) above together with

(10) $x \odot\left(y_{1}+y_{2}\right)=x \odot y_{1}+x \odot y_{2}+H(x) \bar{\otimes}\left(y_{2} \mid y_{1}\right)_{H}$,

(11) $P(a) \odot y=a \bar{\otimes}(y \mid y)_{H}$.

As proved in [7] the tensor product of square groups is a symmetric monoidal structure on the category of square groups with unit $\mathbb{Z}_{\text {nil }}$ in Definition 2.1.1. The associativity isomorphism

$$
(X \odot Y) \odot Z \cong X \odot(Y \odot Z)
$$

is given by $(x @ y) @ z \mapsto x @(y @ z),(a \bar{\otimes} b) \odot z \mapsto a \bar{\otimes}(b \otimes \Delta(z))$ and $(a \otimes b) \bar{\otimes} c \mapsto$ $a \bar{\otimes}(b \otimes c)$ at the $e$-level and by the associativity isomorphism

$$
\left(X_{e e} \otimes Y_{e e}\right) \otimes Z_{e e} \cong X_{e e} \otimes\left(Y_{e e} \otimes Z_{e e}\right)
$$

for the tensor product of abelian groups at the ee-level. The symmetry isomorphism

$$
\tau_{\odot}: X \odot Y \cong Y \odot X
$$

is defined on $e$-groups by $x \subseteq y \mapsto y \odot x, x \odot y \mapsto y @ x$, and $a \bar{\otimes} b \mapsto b \bar{\otimes} a$, and on $e e$-groups by the standard symmetry isomorphism

$$
\tau_{\otimes}: X_{e e} \otimes Y_{e e} \cong Y_{e e} \otimes X_{e e}
$$

for the tensor product of abelian groups. The unit isomorphism

$$
\mathbb{Z}_{\text {nil }} \odot X \cong X
$$

is defined on $e$-groups by the formulas $n \odot x \mapsto n \cdot x$ and $n \bar{\otimes} a \mapsto n \cdot P(a)$. 


\subsection{Exterior Cup-products}

We will work with the track category Top* of compactly generated pointed spaces. A track category is a category enriched in groupoids, i.e. a 2-category where all 2-morphisms are vertically invertible. See [12] for a reference on 2-categories. A 2-morphism in a track category is also termed a track, and a 2-functor between track categories is called a track functor. Tracks in Top* are homotopy classes of homotopies between pointed maps. The identity track on a pointed map $f: X \rightarrow Y$, also called the trivial track, will be denoted by $0 \square$. We use the symbol $\square$ for the vertical composition, and $F^{\boxminus}$ denotes the vertical inverse of a track $F$. Horizontal composition is denoted by juxtaposition.

The smash product of pointed spaces $X, Y$ in Top* is the quotient space

$$
X \wedge Y=X \times Y / X \vee Y
$$

where the coproduct $X \vee Y$ in Top* admits the canonical inclusion $X \vee Y \subset X \times Y$ to the product. If $\sigma$ is a permutation of $\{1, \ldots, n\}$ the map

$$
\sigma: X_{1} \wedge \cdots \wedge X_{n} \longrightarrow X_{\sigma^{-1}(1)} \wedge \cdots \wedge X_{\sigma^{-1}(n)}
$$

induced by the permutation of coordinates according to $\sigma$, is also denoted by $\sigma$. For the sake of simplicity we will occasionally omit the permutation $\sigma$ in the diagrams and equations where it is understood. Given a subset $\left\{i_{1}, \ldots, i_{k}\right\} \subset\{1, \ldots, n\}$ we denote by $\sigma=\left(i_{1} \ldots i_{k}\right)$ the permutation defined by $\sigma\left(i_{s}\right)=i_{s+1}$ if $1 \leq s<k, \sigma\left(i_{k}\right)=i_{1}$ and $\sigma(m)=m$ otherwise.

The smash product is a track functor

$$
\wedge: \text { Top }^{*} \times \text { Top }^{*} \longrightarrow \text { Top* }
$$

It is defined as usually at the level of pointed spaces and pointed maps. The pointed space $I_{+}$is the disjoint union of the interval $I=[0,1]$ with an outer base point $*$. Let $F: f \Rightarrow g$ be a track between maps $f, g: A \rightarrow B$ represented by a homotopy $F: I_{+} \wedge$ $A \rightarrow B$, and let $h: X \rightarrow Y$ be another map. Then the track $F \wedge h: f \wedge h \Rightarrow g \wedge h$ is represented by a homotopy $F \wedge h: I_{+} \wedge A \wedge X \rightarrow B \wedge Y$ and $h \wedge F: h \wedge f \Rightarrow h \wedge g$ is represented by the composite

$$
I_{+} \wedge X \wedge A \cong X \wedge I_{+} \wedge A \stackrel{h \wedge F}{\longrightarrow} Y \wedge B .
$$

If $G: h \Rightarrow k$ is another track then

$$
F \wedge G=(g \wedge G) \square(F \wedge h)=(F \wedge k) \square(f \wedge G): f \wedge h \Rightarrow g \wedge k .
$$

The smash product defines a symmetric monoidal structure in the category Top*. The unit object is the 0 -sphere $S^{0}$.

Definition 2.3.1 Given maps $f: \Sigma A \rightarrow \Sigma B$ and $g: \Sigma X \rightarrow \Sigma Y$ the left exterior cup-product $f \# g$ is the composite

$$
S^{1} \wedge A \wedge X \stackrel{f \wedge X}{\longrightarrow} S^{1} \wedge B \wedge X \cong B \wedge S^{1} \wedge X \stackrel{B \wedge g}{\longrightarrow} B \wedge S^{1} \wedge Y \cong S^{1} \wedge B \wedge Y .
$$


Similarly the right exterior cup-product $f \# g$ is the composite

$$
S^{1} \wedge A \wedge X \cong A \wedge S^{1} \wedge X \stackrel{A \wedge g}{\longrightarrow} A \wedge S^{1} \wedge Y \cong S^{1} \wedge A \wedge Y \stackrel{f \wedge Y}{\longrightarrow} S^{1} \wedge B \wedge Y .
$$

The equality

$$
f \# g=\left(\begin{array}{lll}
2 & 3
\end{array}\right)(g \# f)\left(\begin{array}{ll}
2 & 3
\end{array}\right)
$$

is always satisfied.

These constructions give rise to homotopy operations called exterior cup-products $\#, \#:[\Sigma A, \Sigma B] \times[\Sigma X, \Sigma Y] \longrightarrow[\Sigma A \wedge X, \Sigma B \wedge Y]$

see [1, II.1.14]. The operation \# is left-linear and \# is right-linear,

$$
\begin{aligned}
& \left(f_{1}+f_{2}\right) \# g=f_{1} \# g+f_{2} \# g, \\
& f \underline{\#}\left(g_{1}+g_{2}\right)=f \underline{\#} g_{1}+f \underline{\#} g_{2} .
\end{aligned}
$$

Given a pointed discrete set $E$ we denote $\vee_{E} S^{n}=\Sigma^{n} E$. Then

$$
\pi_{1}\left(\vee_{E} S^{1}\right)=\langle E\rangle
$$

is the free group with basis $E-*$, and

$$
\pi_{n}\left(\vee_{E} S^{n}\right)=\mathbb{Z}[E]
$$

is the free abelian group with basis $E-*$ for $n \geq 2$. We write

$$
\langle E\rangle_{\text {nil }}
$$

for the free group of nilpotency class 2 (nil-group for short) with basis $E-*$, which is obtained from $\langle E\rangle$ by dividing out triple commutators.

If $A=X=S^{0}$, and $B=E, Y=\bar{E}$ are pointed sets then the exterior cup-products are functions

$$
\#, \underline{\#}:\langle E\rangle \times\langle\bar{E}\rangle \longrightarrow\langle E \wedge \bar{E}\rangle \text {. }
$$

These functions factor in a unique way through the natural projection onto the nilization in the following way

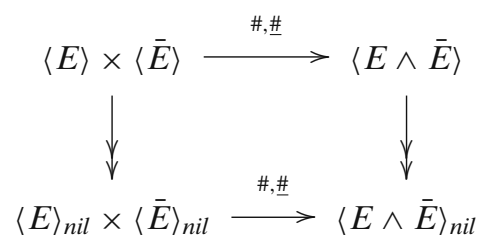


A free nil-group $\langle E\rangle_{\text {nil }}$ on a pointed set $E$ gives rise to a square group

$$
\mathbb{Z}_{\text {nil }}[E]=\left(\langle E\rangle_{\text {nil }} \underset{H}{\stackrel{P}{\leftrightarrows}} \otimes^{2} \mathbb{Z}[E]\right)
$$

defined by $P(a \otimes b)=[b, a], H(e)=0$ for any $e \in E$ and $(s \mid t)_{H}=t \otimes s$ so that $\mathbb{Z}_{\text {nil }}\left[S^{0}\right]=\mathbb{Z}_{\text {nil }}$ in Definition 2.1.1. These square groups are the main examples of good square groups in the sense of Definition 2.1.1. For Eq. 10 the involution $T$ is up to sign the interchange of factors in the tensor square $T(a \otimes b)=-b \otimes a$ and $\Delta$ is defined by $\Delta(e)=e \otimes e$ for $e \in E$. Recall that we denote

$$
\tau_{\otimes}: A \otimes B \cong B \otimes A,
$$

the symmetry isomorphism for the tensor product of abelian groups, which should not be confused with $T=-\tau_{\otimes}$ in this case.

The next proposition is essentially [7,34]. It shows the connection between the tensor product of square groups and the exterior cup-products.

Proposition 2.3.2 Given two pointed sets $E$ and $\bar{E}$ there is a square group isomorphism

$$
\mathbb{Z}_{\text {nil }}[E] \odot \mathbb{Z}_{\text {nil }}[\bar{E}] \stackrel{\cong}{\longrightarrow} \mathbb{Z}_{\text {nil }}[E \wedge \bar{E}]
$$

defined on the e-groups by $x \subseteq y \mapsto x \# y, x \odot y \mapsto x \# y$, and on the ee-groups by

$1 \otimes \tau_{\otimes} \otimes 1: \mathbb{Z}[E] \otimes \mathbb{Z}[E] \otimes \mathbb{Z}[\bar{E}] \otimes \mathbb{Z}[\bar{E}] \cong \mathbb{Z}[E] \otimes \mathbb{Z}[\bar{E}] \otimes \mathbb{Z}[E] \otimes \mathbb{Z}[\bar{E}]$

$a \otimes b \otimes c \otimes d \mapsto a \otimes c \otimes b \otimes d$.

This is the quadratic analogue of the well-known fact that free abelian groups have the tensor product

$$
\mathbb{Z}[E] \otimes \mathbb{Z}[\bar{E}] \cong \mathbb{Z}[E \wedge \bar{E}]
$$

\subsection{The Symmetric Monoidal Category of Quadratic Pair Modules}

A pair in a category $\mathbf{C}$ is a morphism $f: X \rightarrow Y$ in $\mathbf{C}$. Let Pair $(\mathbf{C})$ be the category of such pairs. Morphisms $(\alpha, \beta): f \rightarrow f^{\prime}$ in Pair $(\mathbf{C})$ are given by morphisms $\alpha: X \rightarrow X^{\prime}$ and $\beta: Y \rightarrow Y^{\prime}$ in $\mathbf{C}$ satisfying $\beta f=f^{\prime} \alpha$. A quadratic pair module is a special pair in the category SG of square groups and the category $\mathbf{q p m}$ of quadratic pair modules is a full reflective subcategory of Pair(SG). The left adjoint to the inclusion $\mathbf{q p m} \subset$ Pair(SG), i.e. the reflection functor, is denoted by

$$
\Phi: \text { Pair }(\mathbf{S G}) \longrightarrow \text { qpm. }
$$

Given a pair $f: D \rightarrow C$ in SG we have $\Phi(f)_{(0)}=C$, so that $\Phi(f)_{0}=C_{e}$ and $\Phi(f)_{e e}=C_{e e}$. Moreover, $\Phi(f)_{1}$ is the quotient group

$$
\begin{aligned}
\Phi(f)_{1} & =D_{e} \times C_{e e} / \sim, \\
\left(0, f_{e e}(d)\right) & \sim(P(d), 0), \quad d \in D_{e e}, \\
(0, H P(c)) & \sim(0,2 c), \quad c \in C_{e e} .
\end{aligned}
$$


The operators $P$ and $H$ for $\Phi(f)_{(1)}$ and the homomorphism $\partial: \Phi(f)_{1} \rightarrow \Phi(f)_{0}=$ $C_{e}$ are defined by the formulas, $c \in C_{e e}, d \in D_{e}$,

$$
\begin{aligned}
P(c) & =(0, c), \\
H(d, c) & =f_{e e} H(d)+H P(c), \\
\partial(d, c) & =f_{e}(d)+P(c) .
\end{aligned}
$$

The unit of this adjunction is a natural morphism in Pair(SG)

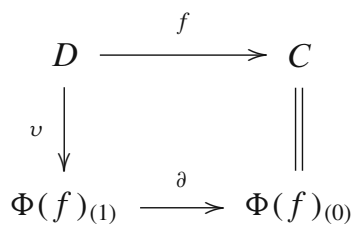

and is given by $v_{e}(d)=(d, 0)$ for $d \in D_{e}$ and $v_{e e}\left(d^{\prime}\right)=f_{e e}\left(d^{\prime}\right)$ for $d^{\prime} \in D_{e e}$. We use the functor $\Phi$ for the following definition of the tensor product in $\mathbf{q p m}$.

The category $\mathbf{q p m}$ is a symmetric monoidal category. This structure is inherited from the tensor product in SG described above. More precisely, the tensor product $C \odot D$ of two quadratic pair modules $\partial: C_{(1)} \rightarrow C_{(0)}, \partial: D_{(1)} \rightarrow D_{(0)}$ is given as follows. Consider the push-out diagram

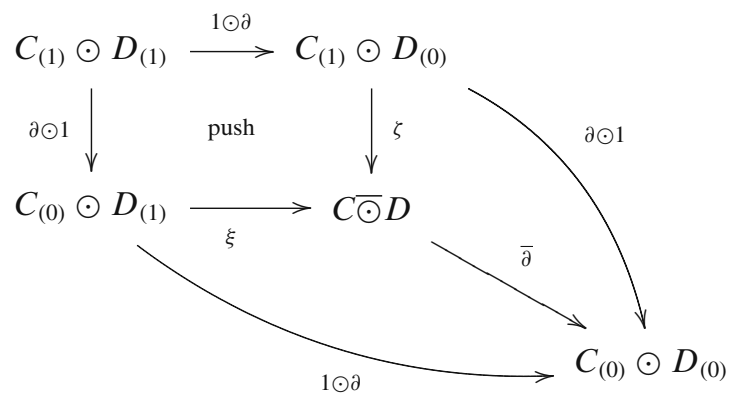

in the category SG. Here $\bar{\partial}$ is a pair in SG for which we derive the tensor product in qpm by the functor $\Phi$ above, that is,

$$
C \odot D=\Phi\left(\bar{\partial}: C \odot D \rightarrow C_{(0)} \odot D_{(0)}\right),
$$

is particular $(C \odot D)_{(0)}=C_{(0)} \odot D_{(0)}$ and $(C \odot D)_{e e}=C_{e e} \otimes D_{e e}$. Moreover, notice that $v \zeta$ and $v \xi$ are both the identity on ee-groups. The unit element for this tensor product is the quadratic pair module $\overline{\mathbb{Z}}_{n i l}=\Phi\left(0 \rightarrow \mathbb{Z}_{\text {nil }}\right)$ given by

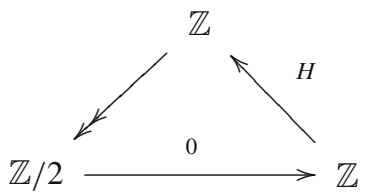

where $H(n)=\left(\begin{array}{l}n \\ 2\end{array}\right)$.

Springer 
2.5 The Track Category of Quadratic Pair Modules

Given a quadratic pair module $C$ the group $C_{0}$ acts on $C_{1}$ by the formula, $x \in C_{1}$, $y \in C_{0}$,

$$
x^{y}=x+P(\partial(x) \mid y)_{H},
$$

see Remark 2.1.3. We define tracks in $\mathbf{q p m}$ as follows.

Definition 2.5.1 A track $\alpha: f \Rightarrow g$ between two morphisms $f, g: C \rightarrow D$ in $\mathbf{q p m}$ is a function

$$
\alpha: C_{0} \longrightarrow D_{1}
$$

satisfying the equations, $x, y \in C_{0}, z \in C_{1}$,

(1) $\alpha(x+y)=\alpha(x)^{f_{0}(y)}+\alpha(y)$,

(2) $g_{0}(x)=f_{0}(x)+\partial \alpha(x)$,

(3) $g_{1}(z)=f_{1}(z)+\alpha \partial(z)$.

These tracks are pulled back from the track category of crossed modules along the forgetful functor in Remark 2.1.3. The track structure for crossed modules is described in $[9,7]$. In particular we obtain the following result.

Proposition 2.5.2 The category is a qpm track category.

The vertical composition of tracks $\alpha, \beta$ is defined by addition $(\alpha \square \beta)(x)=\beta(x)+$ $\alpha(x)$. The horizontal composition of a track $\alpha$ and a map $f, g$ is defined as $(f \alpha)(x)=$ $f_{1} \alpha(x)$ and $(\alpha g)(x)=\alpha g_{0}(x)$. A trivial track $0_{f}^{\square}: f \Rightarrow f$ is always defined as $0_{f}^{\square}(x)=0$.

One can use the interval quadratic pair module II to characterize tracks in qpm in some cases. This quadratic pair module $\mathbb{I}$ is defined as follows.

$$
\mathbb{I}=\left(\begin{array}{ccc}
\otimes^{2} \mathbb{Z}\left[i_{0}, i_{1}\right] \\
\mathbb{Z} \bar{\imath} \oplus \mathbb{Z} / 2 P\left(i_{0} \mid i_{0}\right)_{H} \oplus \mathbb{Z} P\left(i_{0} \mid i_{1}\right)_{H} & >\left\langle i_{0}, i_{1}\right\rangle_{\text {nil }}
\end{array}\right)
$$

The quadratic map $H$ is defined as in Eq. 10. The structure homomorphisms $P$ and $\partial$ are completely determined by the laws of a quadratic pair module and the equality $\partial(\bar{l})=-i_{0}+i_{1}$. There are two obvious inclusions $i_{0}, i_{1}: \overline{\mathbb{Z}}_{n i l} \rightarrow \mathbb{I}$ and a projection $p: \mathbb{I} \rightarrow \overline{\mathbb{Z}}_{n i l}$ defined by $p\left(i_{0}\right)=p\left(i_{1}\right)=1$ and $p(\bar{l})=0$.

Lemma 2.5.3 Let $f, g: C \rightarrow D$ be morphisms in qpm. Assume that $C$ is 0-good. Then tracks $\alpha: f \Rightarrow g$ are in bijection with morphisms $\bar{\alpha}: \mathbb{I} \odot C \rightarrow D$ with $f=\bar{\alpha} i_{0}$ and $g=$ $\bar{\alpha} i_{1}$. The equivalence is given by the formula $\alpha(c)=\bar{\alpha} v_{e} \zeta_{e}(\bar{l} \odot c)$ for $c \in C_{0}$. Here we use the square group morphisms $v$ and $\zeta$ in Eqs. 11 and 12.

This lemma can be derived from the definition of the tensor product of square groups. 
Proposition 2.5.4 The tensor product functor

$$
\odot: \mathbf{q p m} \times \mathbf{q p m} \longrightarrow \mathbf{q p m}
$$

is a track functor.

Proof Given tracks $\alpha: f \Rightarrow g: C \rightarrow D$ and $\beta: h \Rightarrow k: X \rightarrow Y$ in qpm, the track $\alpha \odot \beta: f \odot h \Rightarrow g \odot k: C \odot X \rightarrow D \odot Y$ in qpm with

$$
\alpha \odot \beta:(C \odot X)_{0}=\left(C_{(0)} \odot X_{(0)}\right)_{e} \longrightarrow(D \odot Y)_{1}
$$

is defined as follows. Given $c \in C_{0}$ and $x \in X_{0}$

$$
\begin{aligned}
(\alpha \odot \beta)(c \varrho x)= & v_{e} \xi_{e}\left(f_{0}(c) @ \beta(x)\right)+v_{e} \zeta_{e}\left(\alpha(c) @ k_{0}(x)\right) \\
& +\left(-f_{0}+g_{0} \mid f_{0}(c)\right)_{H} \bar{\otimes} H k_{0}(x) \\
\stackrel{(\mathrm{a})}{=} & v_{e} \zeta_{e}\left(\alpha(c) @ h_{0}(x)\right)+v_{e} \xi_{e}\left(g_{0}(c) @ \beta(x)\right) \\
& +\left(-f_{0}(c)+g_{0}(c) \mid f_{0}(c)\right)_{H} \bar{\otimes} H h_{0}(x),
\end{aligned}
$$

and given $a \in C_{e e}$ and $b \in C_{e e}$

$$
(\alpha \odot \beta)(a \bar{\otimes} b)=-f_{e e}(a) \bar{\otimes} h_{e e}(b)+g_{e e}(a) \bar{\otimes} k_{e e}(b) .
$$

Here we use the square group morphisms $v, \zeta$, and $\xi$ in Eqs. 11 and 12. For (a) we use

$$
v_{e} \zeta_{e}(\partial \alpha(c) @ \beta(x))=v_{e} \xi_{e}(\alpha(c) @ \partial \beta(x))
$$

This equality follows from the fact that the square in Eq. 12 commutes. We leave the reader to check that $\alpha \odot \beta$ is indeed a track $f \odot h \Rightarrow g \odot k$ and that the axioms of a track functor are satisfied.

The following commutativity property for the tensor product of tracks holds.

Lemma 2.5.5 Given tracks $\alpha$, $\beta$ in qpm the equation $\tau_{\odot}(\alpha \odot \beta)=(\beta \odot \alpha) \tau_{\odot}$ holds.

The proof is a straightforward but somewhat lengthy computation. One can also use the track functor $\odot$ in Proposition 2.5.4 to show that $\mathbf{q p m}$ is indeed a symmetric monoidal 2-category, compare [11] and [16].

\section{Secondary Homotopy Groups as a Lax Symmetric Monoidal Functor}

In this part we introduce the additive secondary homotopy group as a quadratic pair module. We formulate our main results on the smash product for additive secondary homotopy groups leading to a lax symmetric monoidal functor. We also give applications to unstable cup-one products, Toda brackets, and secondary Whitehead products. 
3.1 Homotopy Groups and Secondary Homotopy Groups

Let $\mathbf{A b}$ be the category of abelian groups. Using classical homotopy groups $\pi_{n} X$ we obtain for $n \geq 0$ the functor

$$
\Pi_{n}: \text { Top }^{*} \longrightarrow \mathbf{A b}
$$

with

$$
\Pi_{n} X= \begin{cases}\pi_{n} X, & n \geq 2, \\ \left(\pi_{1} X\right)_{a b}, & n=1, \\ \mathbb{Z}\left[\pi_{0} X\right], & n=0,\end{cases}
$$

termed additive homotopy group.

One readily checks that the smash product $f \wedge g: S^{n+m} \longrightarrow X \wedge Y$ of maps $\left\{f: S^{n} \rightarrow X\right\} \in \pi_{n} X$ and $\left\{g: S^{m} \rightarrow Y\right\} \in \pi_{m} Y$ induces a well-defined homomorphism

$$
\wedge: \Pi_{n} X \otimes \Pi_{m} Y \longrightarrow \Pi_{n+m}(X \wedge Y) .
$$

This homomorphism is symmetric in the sense that the symmetry isomorphism $\tau_{\wedge}: X \wedge Y \rightarrow Y \wedge X$ yields the equation in $\Pi_{n+m}(Y \wedge X)$

$$
\left(\tau_{\wedge}\right)_{*}(f \wedge g)=(-1)^{n m} g \wedge f .
$$

The sign $(-1)^{n m}$ is given by the degree of the symmetry isomorphism

$$
\tau_{n, m}=\tau_{\wedge}: S^{n+m}=S^{n} \wedge S^{m} \longrightarrow S^{m} \wedge S^{n}=S^{m+n} .
$$

Here

$$
\tau_{n, m} \in \operatorname{Sym}(n+m)
$$

is the shuffle permutation of $n+m$ elements which exchanges the blocks $\{1, \ldots, n\}$ and $\{n+1, \ldots, n+m\}$. For this we recall that the symmetric group of $k$ letters $\operatorname{Sym}(k)$ acts on the $k$-sphere

$$
S^{k}=S^{1} \wedge \cdots \wedge S^{1}
$$

by permutation of coordinates according to Eq. 7 .

The main purpose of this paper is the generalization of the smash product operator (15) for additive secondary homotopy groups.

Definition 3.1.1 Let $n \geq 2$. For a pointed space $X$ we define the additive secondary homotopy group $\Pi_{n, *} X$ which is the quadratic pair module given by the diagram

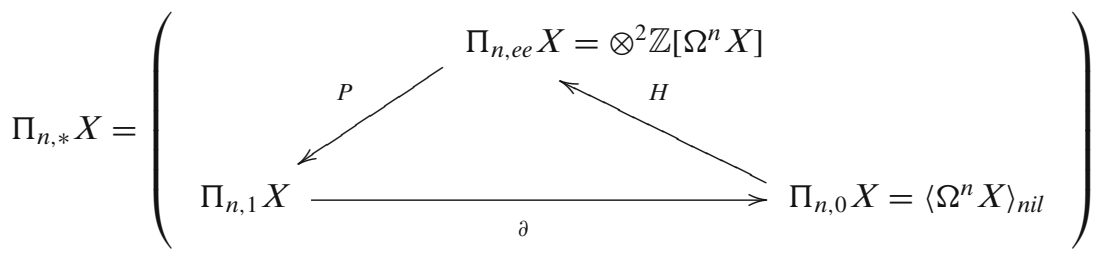

Here $\Omega^{n} X$ is the discrete pointed set of maps $S^{n} \rightarrow X$ in Top* and $H$ is defined as in Eq. 10. 
We describe the group $\Pi_{n, 1} X$ and the homomorphisms $P$ and $\partial$ as follows. The group $\Pi_{n, 1} X$ is given by the set of equivalence classes $[f, F]$ represented by a map $f: S^{1} \rightarrow \vee_{\Omega^{n} X} S^{1}$ and a track

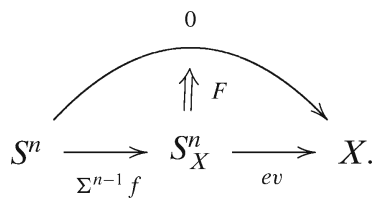

Here the pointed space

$$
S_{X}^{n}=\vee_{\Omega^{n} X} S^{n}=\Sigma^{n} \Omega^{n} X
$$

is the $n$-fold suspension of the discrete pointed set $\Omega^{n} X$, i.e. $S_{X}^{n}$ is the coproduct of $n$-spheres indexed by the set of non-trivial maps $S^{n} \rightarrow X$. The map ev: $S_{X}^{n} \rightarrow X$ is the obvious evaluation map. Given a map $f: S^{1} \rightarrow \vee_{\Omega^{n} X} S^{1}$ we will denote $f_{e v}=$ $e v\left(\Sigma^{n-1} f\right)$, so that $F$ in the previous diagram is a track $F: f_{e v} \Rightarrow 0$.

The equivalence relation $[f, F]=[g, G]$ holds provided there is a track

$$
N: \Sigma^{n-1} f \Rightarrow \Sigma^{n-1} g
$$

with $\overline{\operatorname{Hopf}}(N)=0$, in the sense of Eq. 21 below, such that the composite track in the following diagram is the trivial track.

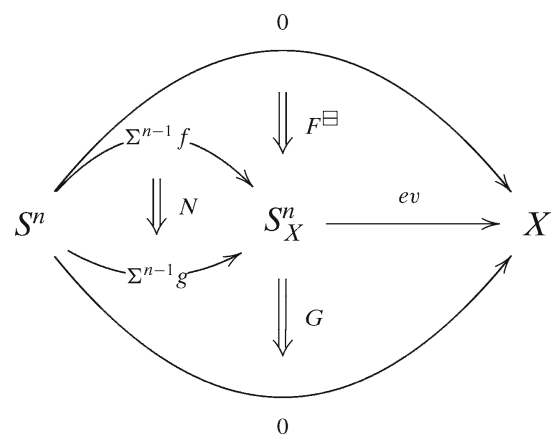

That is $F=G \square(e v N)$. The map $\partial$ is defined by the formula

$$
\partial[f, F]=\left(\pi_{1} f\right)_{n i l}(1),
$$

where $1 \in \pi_{1} S^{1}=\mathbb{Z}$.

The Hopf invariant Hopf $(N)$ of a track $N: \Sigma^{n-1} f \Rightarrow \Sigma^{n-1} g$ between maps as above is defined in $[9,3.3]$ by the homomorphism

$$
H_{2}\left(I S^{1}, S^{1} \vee S^{1}\right) \stackrel{\operatorname{ad}(N)_{*}}{\longrightarrow} H_{2}\left(\Omega^{n-1} S_{X}^{n}, \vee_{\Omega^{n} X} S^{1}\right) \cong\left\{\begin{array}{l}
\hat{\otimes}^{2} \mathbb{Z}\left[\Omega^{n} X\right], n \geq 3, \\
\otimes^{2} \mathbb{Z}\left[\Omega^{n} X\right], n=2 .
\end{array}\right.
$$

which carries the generator $1 \in \mathbb{Z} \cong H_{2}\left(I S^{1}, S^{1} \vee S^{1}\right)$ to $\operatorname{Hopf}(N)$. Here the isomorphism is induced by the Pontrjagin product and $\operatorname{ad}(N)_{*}$ is the homomorphism induced in homology by the adjoint of

$$
S^{n-1} \wedge I_{+} \wedge S^{1} \stackrel{(12)}{\cong} I_{+} \wedge S^{n} \stackrel{N}{\rightarrow} S_{X}^{n}
$$


The reduced tensor square $\hat{\otimes}^{2}$ in Eq. 20 is given by

$$
\hat{\otimes}^{2} A=\frac{A \otimes A}{a \otimes b+b \otimes a \sim 0},
$$

and $\bar{\sigma}: \otimes^{2} A \rightarrow \hat{\otimes}^{2} A$ is the natural projection, with $\bar{\sigma}(a \otimes b)=a \hat{\otimes} b$. We define

$$
\overline{H o p f}=\left\{\begin{array}{l}
\text { Hopf, } \text { for } n \geq 3, \\
\bar{\sigma} \text { Hopf, for } n=2 .
\end{array}\right.
$$

We refer the reader to [9, 3] for the elementary properties of Hopf which will be used in this paper.

This completes the definition of $\Pi_{n, 1} X, n \geq 2$, as a set. The group structure of $\Pi_{n, 1} X$ is induced by the comultiplication $\mu: S^{1} \rightarrow S^{1} \vee S^{1}$, compare [9, 4.4].

We now define the homomorphism $P$. Consider the diagram

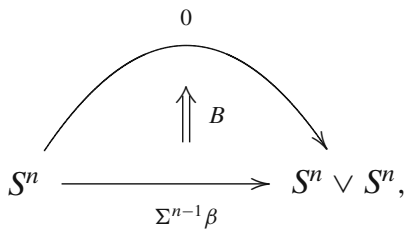

where $\beta: S^{1} \rightarrow S^{1} \vee S^{1}$ is given such that $\left(\pi_{1} \beta\right)_{\text {nil }}(1)=[a, b] \in\langle a, b\rangle_{\text {nil }}$ is the commutator of the generators. The track $B$ is any track with $\overline{\operatorname{Hopf}}(B)=-a \hat{\otimes} b \in \hat{\otimes}^{2} \mathbb{Z}[a, b]$. Given $x \otimes y \in \otimes^{2} \mathbb{Z}\left[\Omega^{n} X\right]$ let $\tilde{x}, \tilde{y}: S^{1} \rightarrow \vee_{\Omega^{n} X} S^{1}$ be maps with $\left(\pi_{1} \tilde{x}\right)_{a b}(1)=x$ and $\left(\pi_{1} \tilde{y}\right)_{a b}(1)=y$. Then the diagram

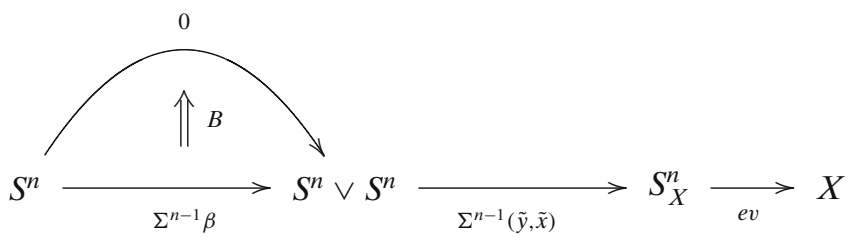

represents an element

$$
P(x \otimes y)=\left[(\tilde{y}, \tilde{x}) \beta,\left(\tilde{y}_{e v}, \tilde{x}_{e v}\right) B\right] \in \Pi_{n, 1} X .
$$

This completes the definition of the quadratic pair module $\Pi_{n, *} X$ for $n \geq 2$. For $n=0,1$ we define the additive secondary homotopy groups $\Pi_{n, *} X$ by Remark 3.1.3 below. In this way we get for $n \geq 0$ a functor

$$
\Pi_{n, *}: \text { Top }^{*} \longrightarrow \mathbf{q p m}
$$

which is actually a track functor. 
There homology of the additive secondary homotopy groups is given by

$$
\begin{aligned}
& h_{0} \Pi_{n, *} X \cong \pi_{n} X, \quad n \geq 2, \\
& h_{1} \Pi_{n, *} X \cong \pi_{n+1} X, \quad n \geq 3, \\
& h_{1} \Pi_{2, *} X \cong \pi_{3} X /\left[\pi_{2} X, \pi_{2} X\right],
\end{aligned}
$$

where $[-,-]$ denotes here the Whitehead product. For this we use $[9,5.1]$, and also $[9,6.11]$ for the case $n=2$. Furthermore, the following property is crucial.

\section{Proposition 3.1.2 The homomorphism}

$$
h_{0} \Pi_{n, *} X \rightarrow h_{1} \Pi_{n, *} X: x \mapsto P(x \mid x)_{H}
$$

coincides via Eq. 23 with the homomorphism $\eta^{*}: \pi_{n} X \otimes \mathbb{Z} / 2 \rightarrow \pi_{n+1} X$ if $n \geq 3$ and $\eta^{*}: \pi_{2} X \otimes \mathbb{Z} / 2 \rightarrow \pi_{3} X /\left[\pi_{2} X, \pi_{2} X\right]$ if $n=2$. Here $\eta^{*}$ is induced by precomposition with $\Sigma^{n-2} \eta$, where $\eta: S^{3} \rightarrow S^{2}$ is the Hopf map.

This follows from $[9,8.2]$.

Remark 3.1.3 Considering maps $f: S^{n} \rightarrow X$ together with tracks of such maps to the trivial map, we introduced in [9] the secondary homotopy group $\pi_{n, *} X$, which is a groupoid for $n=0$, a crossed module for $n=1$, a reduced quadratic module for $n=2$, and a stable quadratic module for $n \geq 3$. The categories formed by these algebraic objects are related by forgetful functors

$$
\left(\begin{array}{c}
\text { stable } \\
\text { quadratic } \\
\text { modules }
\end{array}\right) \stackrel{\phi_{3}}{\longrightarrow}\left(\begin{array}{c}
\text { reduced } \\
\text { quadratic } \\
\text { modules }
\end{array}\right) \stackrel{\phi_{2}}{\longrightarrow}\left(\begin{array}{c}
\text { crossed } \\
\text { modules }
\end{array}\right) \stackrel{\phi_{1}}{\longrightarrow} \text { (groupoids) . }
$$

Then using the left adjoint functors $\mathrm{Ad}_{n}$ of the forgetful functors $\phi_{n}$ as discussed in $[9,6]$ we get the additive secondary homotopy group track functor

$$
\Pi_{n, *}: \text { Top }^{*} \longrightarrow \text { squad }
$$

given by

$$
\Pi_{n, *} X= \begin{cases}\pi_{n, *} X, & \text { for } n \geq 3, \\ \operatorname{Ad}_{3} \pi_{2, *} X, & \text { for } n=2, \\ \operatorname{Ad}_{3} \operatorname{Ad}_{2} \pi_{1, *} X, & \text { for } n=1 \\ \operatorname{Ad}_{3} \operatorname{Ad}_{2} \operatorname{Ad}_{1} \pi_{0, *} X, & \text { for } n=0\end{cases}
$$

This is the secondary analogue of Eq. 14.

The category squad of stable quadratic modules is not appropriate to study the smash product of secondary homotopy groups since we do not have a symmetric monoidal structure in squad. Therefore we introduced above the category $\mathbf{q p m}$ of quadratic pair modules and we observe that $\Pi_{n, *} X$ in squad yields a functor to the category $\mathbf{q p m}$ in the following way. As a stable quadratic module $\Pi_{n, *} X$ looks as follows

$$
\otimes^{2} \mathbb{Z}\left[\Omega^{n} X\right]=\otimes^{2}\left(\Pi_{n, 0} X\right)_{a b} \stackrel{P(-\mid-)_{H}}{\longrightarrow} \Pi_{n, 1} X \stackrel{\partial}{\longrightarrow} \Pi_{n, 0} X=\left\langle\Omega^{n} X\right\rangle_{n i l} .
$$

In the quadratic pair module $\Pi_{n, *} X$ the quadratic map $H$ is defined as in Eq. 10 and the homomorphism $(-\mid-)_{H}: \otimes^{2}\left(\Pi_{n, 0} X\right)_{a b}=\Pi_{n, e e} X$ is the identity. A map 
$f: X \rightarrow Y$ in Top* induces a homomorphism $\Pi_{n, 0} f: \Pi_{n, 0} X \rightarrow \Pi_{n, 0} Y$ between free nil-groups. This homomorphism carries generators in $\Pi_{n, 0} X$ to generators in $\Pi_{n, 0} Y$ and therefore $\Pi_{n, *} f$ is compatible with $H$. This shows that there is a canonical lift

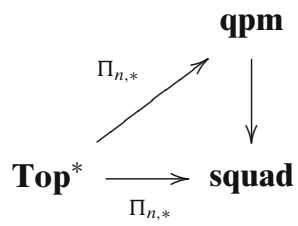

with $\Pi_{n,(0)} X=\mathbb{Z}_{n i l}\left[\Omega^{n} X\right]$ for all $n \geq 0$. In particular $\Pi_{n, *} X$ is always a 0 -good quadratic pair module. Compare [10,1.15].

The definition of $\Pi_{2, *} X$ given above coincides with the lifting of $\operatorname{Ad}_{3} \pi_{2, *} X$ to $\mathbf{q p m}$ by the claim (*) in the proof of $[9,4.9]$.

Generalizing Eq. 23 we have for all $n \geq 0$ a natural isomorphism

$$
h_{0} \Pi_{n, *} X \cong \Pi_{n} X
$$

see $[9,6.10]$.

In this paper we are concerned with the properties of the track functor $\Pi_{n, *}$ mapping to the category $\mathbf{q p m}$. In order to simplify notation given a map $f: X \rightarrow Y$ in Top* we will just denote

$$
f_{*}=\Pi_{n, i} f: \Pi_{n, i} X \longrightarrow \Pi_{n, i} Y, \text { for } i \in\{0,1, e e\} \text { and } n \geq 0 .
$$

Moreover, given a track $\alpha: f \Rightarrow g$ between maps $f, g: X \rightarrow Y$ we denote by

$$
\alpha_{*}=\Pi_{n, *} \alpha: \Pi_{n, 0} X \longrightarrow \Pi_{n, 1} Y, \quad n \geq 0,
$$

the induced track $\alpha_{*}: f_{*} \Rightarrow g_{*}$ in $\mathbf{q p m}$.

\subsection{Smash Product for Secondary Homotopy Groups}

In this section we describe our main results connecting the tensor product of quadratic pair modules and the smash product of pointed spaces. The smash product operator in the next theorem is the canonical analogue of the smash product for classical homotopy groups in Eq. 15 above.

Theorem 3.2.1 The functor of additive secondary homotopy groups $\Pi_{n, *}$ : Top* $\rightarrow$ qpm admits a well-defined smash product operator

$$
\wedge: \Pi_{n, *} X \odot \Pi_{m, *} Y \longrightarrow \Pi_{n+m, *}(X \wedge Y),
$$

which is a morphism in $\mathbf{q p m}$, inducing the smash product of classical homotopy groups in Eq. 15 on $h_{0}$. This operator is natural in $X$ and $Y$ with respect to maps and tracks.

This smash product operator is given in the (0)-level by the following morphism of square groups

$$
\mathbb{Z}_{\text {nil }}\left[\Omega^{n} X\right] \odot \mathbb{Z}_{\text {nil }}\left[\Omega^{m} Y\right] \cong \mathbb{Z}_{\text {nil }}\left[\Omega^{n} X \wedge \Omega^{m} Y\right] \stackrel{\mathbb{Z}_{\text {nil }}[\wedge]}{\longrightarrow} \mathbb{Z}_{\text {nil }}\left[\Omega^{n+m}(X \wedge Y)\right] .
$$


Here

$$
\wedge:\left(\Omega^{n} X\right) \wedge\left(\Omega^{m} Y\right) \longrightarrow \Omega^{m+n}(X \wedge Y), \quad n, m \geq 0,
$$

is the map between discrete pointed sets defined by

$$
\left(f: S^{n} \rightarrow X\right) \wedge\left(g: S^{m} \rightarrow Y\right) \mapsto\left(f \wedge g: S^{n+m} \rightarrow X \wedge Y\right)
$$

On the (1)-level the definition of the smash product operator in Theorem 3.2.1 is more elaborate, see Definition 4.3.3 below.

Proof of Theorem 3.2.1 The first part of Theorem 3.2.1 follows from Lemmas 4.7.1, 4.7.2, 4.7.3 and 4.8.1 in Section 3. For the naturality we use Lemma 4.8.2 in Section 4 and Theorems 3.2.2 and 3.2.3.

We will use the following notation for the image of generators in the tensor product by the smash product morphism in Theorem 3.2.1. Given $x \in \Pi_{n, i} X$ and $y \in \Pi_{m, j} Y$ for $0 \leq i, j, i+j \leq 1$ we denote by

$$
x \wedge y \in \Pi_{n+m, i+j}(X \wedge Y)
$$

the image by $\wedge$ of the element $x \odot y \in \Pi_{n+m, 0}(X \wedge Y)$ if $i=j=0$, of $v_{e} \zeta_{e}(x \odot y) \in$ $\Pi_{n+m, 1}(X \wedge Y)$ if $i=1$ and $j=0$, and of $v_{e} \xi_{e}(x \odot y) \in \Pi_{n+m, 1}(X \wedge Y)$ if $i=0$ and $j=1$. Here we use the square group morphisms $v, \zeta$ and $\xi$ in Eqs. 11 and 12. Similarly for $x \odot y$ and $x \wedge y$. Moreover, given $a \in \Pi_{n, e e} X$ and $b \in \Pi_{m, e e} Y$ we denote by

$$
a \wedge b \in \Pi_{n+m, e e}(X \wedge Y)
$$

the image of $a \otimes b \in\left(\Pi_{n, *} X \odot \Pi_{m, *} Y\right)_{e e}$ by $\wedge$.

Theorem 3.2.2 The smash product operator endows $\Pi_{*, *}$ with the structure of a lax monoidal functor from Top* to the category of graded 0-good quadratic pair modules. That is, the following diagram commutes

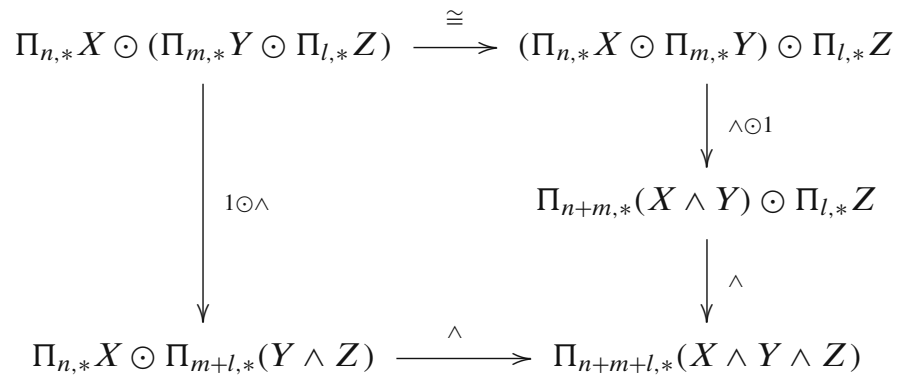


and for the unit $S^{0}$ of the symmetric monoidal category (Top*, $\wedge$ ) and the unit $\overline{\mathbb{Z}}_{\text {nil }}$ of (qpm, $\odot$ ) there is an isomorphism $u: \overline{\mathbb{Z}}_{n i l} \cong \Pi_{0, *} S^{0}$ such that the following diagrams commute.

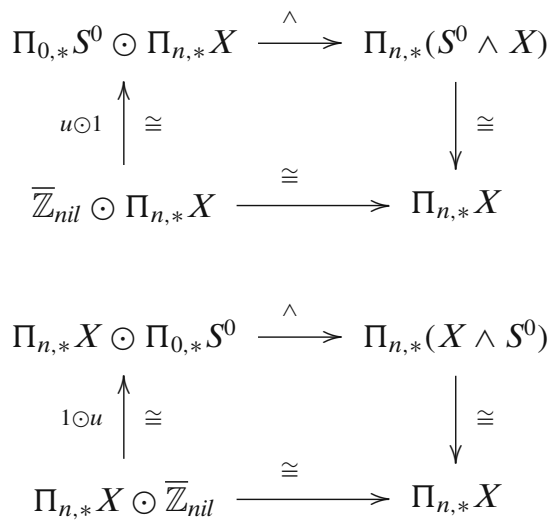

The isomorphism $u: \overline{\mathbb{Z}}_{n i l} \cong \Pi_{0, *} S^{0}$ is the unique one sending $1 \in \mathbb{Z}=\left(\overline{\mathbb{Z}}_{\text {nil }}\right)_{0}$ to $u(1)=1_{S^{0}}: S^{0} \rightarrow S^{0}$ in $\Pi_{0,0} S^{0}$.

Proof of Theorem 3.2.2 In dimensions $\geq 1$ the associativity property in Theorem 3.2.2 follows from Lemma 4.7.4 in Section 4. In case dimension 0 is involved we use the more algebraic Lemma 4.8.1. The commutativity of the squares with the isomorphism $u$ is easy to check. This is left to the reader.

The graded commutativity Eq. 26 for the smash product for classical homotopy groups has a secondary analogue as follows.

Theorem 3.2.3 The following diagram commutes in $\mathbf{q p m}$.

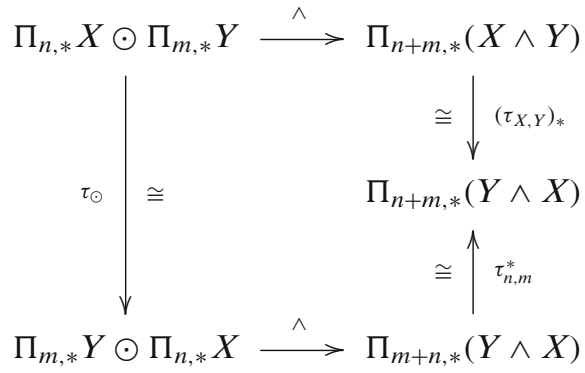

Here $\tau_{\odot}$ is the symmetry isomorphism of the tensor product $\odot$ in $\mathbf{q p m}$, and $\tau_{n, m}^{*}$ is given by the right action of the shuffle permutation $\tau_{n, m} \in \operatorname{Sym}(n+m)$ in Eq. 18 on $\Pi_{m+n, *}(Y \wedge X)$, see the next section.

This follows from Lemma 4.7.5 in Section 4. 
3.3 The Symmetric Action on Smash Products

Secondary homotopy groups, regarded as a functor from pointed spaces to graded 0-good quadratic pair modules

$$
\Pi_{*, *}: \mathbf{T o p}^{*} \longrightarrow \mathbf{q p m}_{0}^{\mathbb{N}},
$$

is a lax monoidal functor, see Theorem 3.2.2. The monoidal structure in $\mathbf{q p m}_{0}^{\mathbb{N}}$ is the usual graded extension of the tensor product $\odot$ of quadratic pair modules, see the appendix, and Top* has the monoidal structure given by the smash product $\wedge$. Both monoidal structures are symmetric, however Theorem 3.2.3 shows that $\Pi_{*, *}$ is not lax symmetric monoidal since the action of the shuffle permutation $\tau_{n, m}$ in Eq. 18 is involved. This inconvenience is solved by enriching the structure of secondary homotopy groups with the sign action of the symmetric track groups constructed in [10].

Definition 3.3.1 Let $\{ \pm 1\}$ be the multiplicative group of order 2. A sign group $G_{\square}$ is a diagram of group homomorphisms

$$
\{ \pm 1\} \stackrel{\iota}{\hookrightarrow} G_{\square} \stackrel{\delta}{\rightarrow} G \stackrel{\varepsilon}{\longrightarrow}\{ \pm 1\}
$$

where the first two morphisms $l$ and $\delta$ form an extension. Here all groups have a multiplicative group law and the composite $\varepsilon \delta$ is also denoted by $\varepsilon: G_{\square} \rightarrow\{ \pm 1\}$.

A morphism $f_{\square}: G_{\square} \rightarrow K_{\square}$ of sign groups is a commutative diagram of group homomorphisms

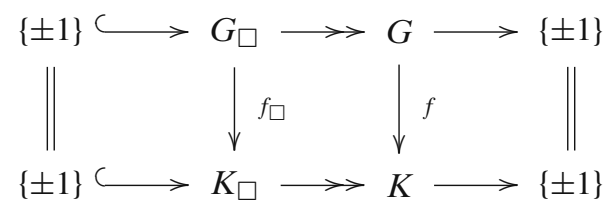

This defines the category $\mathbf{G r}_{ \pm}$of sign groups. The initial object $1_{\square}$ in this category given by $G=\{1\}$ will be termed the trivial sign group.

Remark 3.3.2 Recall from $[10,3.6]$ that a sign group $G_{\square}$ gives rise to a crossed module

$$
\delta_{\square}=(\varepsilon, \delta): G_{\square} \longrightarrow\{ \pm 1\} \times G,
$$

where $\{ \pm 1\} \times G$ acts on $G_{\square}$ by the formula

$$
\left.g^{(x, h)}=\bar{h}^{-1} g \bar{h} l\left(\varepsilon(g)^{(x \cdot \varepsilon(h)}\right)\right) .
$$

Here $g \in G_{\square}, x \in\{ \pm 1\}, h \in G$, and $\bar{h} \in G_{\square}$ is any element with $\delta(\bar{h})=h$. This is a well-defined crossed module since $G_{\square}$ is a central extension of $G$ by $\{ \pm 1\}$.

The main examples of sign groups are the symmetric track groups

$$
\{ \pm 1\} \hookrightarrow \operatorname{Sym}_{\square}(n) \stackrel{\delta}{\rightarrow} \operatorname{Sym}(n) \stackrel{\text { sign }}{\longrightarrow}\{ \pm 1\},
$$


defined as follows. The symmetric group $\operatorname{Sym}(n)$ acts on the left of the $n$-sphere

$$
S^{n}=S^{1} \wedge \stackrel{n}{n} \wedge S^{1},
$$

see Eq. 7. The elements of the symmetric track group $\operatorname{Sym}_{\square}(n)$ for $n \geq 2$ are tracks $\alpha: \sigma \Rightarrow(\cdot)_{n}^{\operatorname{sign}(\sigma)}$ between maps $\sigma,(\cdot)_{n}^{\operatorname{sign}(\sigma)}: S^{n} \rightarrow S^{n}$, with $\sigma \in \operatorname{Sym}(n)$ and $(\cdot)_{n}^{\operatorname{sign}(\sigma)}=$ $\Sigma^{n-1}(\cdot)^{\operatorname{sign}(\sigma)}$, where

$$
(\cdot)^{k}: S^{1} \longrightarrow S^{1}: z \mapsto z^{k}, k \in \mathbb{Z},
$$

is given by the (multiplicative) topological abelian group structure of $S^{1}$. The group law in $\operatorname{Sym}_{\square}(n)$ is given by the horizontal composition of tracks. For $n=0,1$, let $\operatorname{Sym}_{\square}(n)$ be the trivial sign group. Compare [10, 5 and 6].

The smash product $S^{m} \wedge-$ induces a sign group morphism

$$
S^{m} \wedge-: \operatorname{Sym}_{\square}(n) \longrightarrow \operatorname{Sym}_{\square}(m+n),
$$

sending a track $\alpha: \sigma \Rightarrow(\cdot)_{n}^{\operatorname{sign}(\sigma)}$ as above to $S^{m} \wedge \alpha$. This morphism is given on symmetric groups by the usual inclusion $\operatorname{Sym}(n) \subset \operatorname{Sym}(m+n)$, obtained by regarding $\operatorname{Sym}(n)$ as the subgroup of permutations of $m+n$ elements fixing the first $m$ elements.

One can not directly define a sign group morphism

$$
-\wedge S^{n}: \operatorname{Sym}_{\square}(m) \rightarrow \operatorname{Sym}_{\square}(m+n)
$$

in a geometric way as above since $(\cdot)_{m}^{k} \wedge S^{n} \neq(\cdot)_{m+n}^{k}$. With the help of the crossed module structure for sign groups, described in Remark 3.3.2, and the shuffle permutation $\tau_{n, m}$ in Eq. 18, we define $-\wedge S^{n}$ as the following composite

$$
-\wedge S^{n}: \operatorname{Sym}_{\square}(m) \stackrel{S^{n} \wedge-}{\longrightarrow} \operatorname{Sym}_{\square}(n+m) \stackrel{(\cdot)^{\left(1, \tau_{n}, m\right)}}{\longrightarrow} \operatorname{Sym}_{\square}(m+n) .
$$

This morphism is given on symmetric groups by the inclusion $\operatorname{Sym}(m) \subset \operatorname{Sym}(m+n)$, obtained by identifying $\operatorname{Sym}(m)$ with the group of permutations of $m+n$ elements which only permute the first $m$ ones.

Definition 3.3.3 A twisted bilinear morphism of sign groups

$$
\left(f_{\square}, g_{\square}\right): G_{\square} \times L_{\square} \longrightarrow K_{\square}
$$

is given by a pair of sign group morphisms $f_{\square}: G_{\square} \rightarrow K_{\square}, g_{\square}: L_{\square} \rightarrow K_{\square}$, such that the following equations hodl. Given $a \in G$ and $b \in L$ the equality

$$
f(a) g(b)=g(b) f(a)
$$

holds in $K$, and therefore the group homomorphism

$$
(f, g): G \times L \longrightarrow K:(a, b) \mapsto f(a) g(b)
$$

is defined. Moreover, given $x \in G_{\square}, y \in L_{\square}$ the following equality is satisfied in $K_{\square}$

$$
f_{\square}(x) g_{\square}(y)=g_{\square}(y) f_{\square}(x) l\left((-1)^{\left(\begin{array}{c}
\varepsilon(x) \\
2
\end{array}\right)\left(\begin{array}{c}
\varepsilon(y) \\
2
\end{array}\right)}\right) .
$$

The twisted product $G_{\square} \tilde{\times} L_{\square}$ of sign groups $G_{\square}, L_{\square}$ is a sign group

$$
\{ \pm 1\} \stackrel{l}{\hookrightarrow} G_{\square} \tilde{\times} L_{\square} \rightarrow G \times L \stackrel{\varepsilon}{\longrightarrow}\{ \pm 1\}
$$


together with a universal twisted bilinear morphism

$$
\left(i_{G_{\square}}, i_{L_{\square}}\right): G_{\square} \times L_{\square} \longrightarrow G_{\square} \tilde{\times} L_{\square} .
$$

The group $G_{\square} \tilde{x} L_{\square}$ is generated by the symbols $\bar{s}, \bar{t}$ and $\omega$, for $t \in G_{\square}$ and $s \in L_{\square}$, with the following relations:

(1) $\omega$ is central,

(2) $\overline{l(-1)}=\omega$ for both $\imath:\{ \pm 1\} \hookrightarrow G_{\square}$ and $\imath:\{ \pm 1\} \hookrightarrow L_{\square}$,

(3) $\overline{r_{1} r_{2}}=\overline{r_{1}} \cdot \overline{r_{2}}$ when $r_{1}, r_{2}$ lie both in the group $G_{\square}$ or both in $L_{\square}$,

(4) $\bar{t} \bar{s}=\bar{s} \bar{t} \omega^{\left(\begin{array}{c}(t) \\ 2\end{array}\right)\left(\begin{array}{c}\varepsilon^{(s)} \\ 2\end{array}\right)}$ for $t \in G_{\square}$ and $s \in L_{\square}$.

The homomorphism $\delta: G_{\square} \tilde{\times} L_{\square} \rightarrow G \times L$ is defined by $\delta(\bar{t})=(\delta(t), 1)$ for $t \in G_{\square}$, $\delta(\bar{s})=(1, \delta(s))$ for $s \in L_{\square}$, and $\delta(\omega)=1$. The universal bilinear morphism is given by $i_{G_{\square}}(t)=\bar{t}$ and $i_{L_{\square}}(s)=\bar{s}$. The twisted product is a non-symmetric monoidal structure in the category $\mathbf{G r}_{ \pm}$of sign groups where the unit is the trivial sign group $1_{\square}$.

Proposition 3.3.4 The morphisms in Eqs. 25 and 26 induce a morphism of sign groups

$$
\operatorname{Sym}_{\square}(m) \tilde{\times} \operatorname{Sym}_{\square}(n) \longrightarrow \operatorname{Sym}_{\square}(m+n) .
$$

This proposition can be easily derived from the presentation of the symmetric track groups given in [10, 6.11].

We now introduce the action of a sign group on a quadratic pair module. In [10] we show that the sign group $\operatorname{Sym}_{\square}(n)$ acts in this sense on $\Pi_{n, *} X$.

Definition 3.3.5 A sign group $G_{\square}$ acts on the right of a quadratic pair module $C$ if $G$ acts on the right of $C$ by morphisms $g^{*}: C \rightarrow C, g \in G$, in $\mathbf{q p m}$, and there is a bracket

$$
\langle-,-\rangle=\langle-,-\rangle_{G}: C_{0} \times G_{\square} \longrightarrow C_{1}
$$

satisfying the following properties, $x, y \in C_{0}, z \in C_{1}, s, t \in G_{\square}$.

(1) $\langle x+y, t\rangle=\langle x, t\rangle+\langle y, t\rangle+P\left(-\delta(t)^{*}(x)+\varepsilon(t)^{*}(x) \mid \delta(t)^{*}(y)\right)_{H}$,

(2) $\varepsilon(t)^{*}(x)=\delta(t)^{*}(x)+\partial\langle x, t\rangle$,

(3) $\varepsilon(t)^{*}(z)=\delta(t)^{*}(z)+\langle\partial(z), t\rangle$,

(4) $\langle x, s \cdot t\rangle=\left\langle\delta(s)^{*}(x), t\right\rangle+\left\langle\varepsilon(t)^{*}(x), s\right\rangle$,

(5) For the element $\omega=\imath(-1) \in G_{\square}$ we have the $\omega$-formula:

$$
\langle x, \omega\rangle=P(x \mid x)_{H} .
$$

Notice that the $\omega$-formula corresponds to the homomorphism in Proposition 3.2.

Here we use the notation

$-(-1)^{*}(x)=-x+\partial P H(x)$,

$-(-1)^{*}(z)=-y+H P \partial(y)$,

$-1^{*}=$ identity,

introduced in [10]. Notice that $(-1)^{*}(-1)^{*}=1^{*}$. The trivial sign group acts on any quadratic pair module in a unique way.

空 Springer 
This definition of a sign group action, as given in [10], can be reinterpreted in terms of the tensor product $\odot$ of quadratic pair modules. For this we use the following "group ring" construction for sign groups.

Definition 3.3.6 A quadratic pair algebra $R$ is a monoid in the category qpm of quadratic pair modules. The image of a right-linear generator $r \odot s$ in the tensor product by the monoid structure morphism $R \odot R \rightarrow R$ will be denoted by $r \cdot s$. This notation will also be used below (in the proof of Lemma 3.3.8) for right modules over a quadratic pair algebra. Given a sign group $G_{\square}$ the quadratic pair algebra $A\left(G_{\square}\right)$ has generators

- $\quad[g]$ for any $g \in G$ on the 0-level,

- $\quad[t]$ for any $t \in G_{\square}$ on the 1-level,

- No generators on the ee-level,

and relations

- $H[g]=0$ for $g \in G$,

- $\quad[1]=1$ the unit element,

- $\quad[g h]=[g] \cdot[h]$ for $g, h \in G$,

$-\quad \partial[t]=-[\delta(t)]+\varepsilon(t)$,

- $\quad[s t]=[\delta(s)] \cdot[t]+\varepsilon(t) \cdot[s]+\left(\begin{array}{c}\varepsilon(s) \\ 2\end{array}\right)\left(\begin{array}{c}\varepsilon(t) \\ 2\end{array}\right) P(1 \mid 1)_{H}$ for $s, t \in G_{\square}$,

$-\quad[\omega]=P(1 \mid 1)_{H}$, where $\omega=l(-1)$.

In these equations -1 can appear as a value of the homomorphism $\varepsilon$. This -1 denotes the additive opposite of the unit element $1 \in A_{0}\left(G_{\square}\right)$, except when it appears as part of a cominatorial number, where it is regarded just as an integer. The relations above show that $A_{(0)}\left(G_{\square}\right)=\mathbb{Z}_{n i l}\left[G_{+}\right]$where $G_{+}$is the group $G$ together with an outer base point, so $A\left(G_{\square}\right)$ is 0 -good. A more explicit description of the groups and the structure homomorphisms of $A\left(G_{\square}\right)$ as a quadratic pair module can be found in [10, Remark 5.5]. The homology of $A\left(G_{\square}\right)$ is given by

$$
\begin{aligned}
& h_{0} A\left(G_{\square}\right) \cong \mathbb{Z}, \quad \text { with natural projection }\left\langle G_{+}\right\rangle_{\text {nil }} \rightarrow \mathbb{Z}:[g] \mapsto \varepsilon(g), \\
& h_{1} A\left(G_{\square}\right) \cong \mathbb{Z} / 2, \quad \text { generated by }[\omega]=P(1 \mid 1)_{H} .
\end{aligned}
$$

The "group ring" of a sign group defines a functor onto the category qpa of quadratic pair algebras

$$
A: \mathbf{G r}_{ \pm} \longrightarrow \text { qpa }
$$

\section{Proposition 3.3.7 The functor $A$ is strict monoidal.}

Proof Given two sign groups $G_{\square}, L_{\square}$, if we use the notation $\left(i_{G_{\square}}, i_{L_{\square}}\right)$ in Eq. 27 for the universal twisted bilinear morphism then the isomorphism $A\left(G_{\square} \tilde{\times} L_{\square}\right) \cong$ $A\left(G_{\square}\right) \odot A\left(L_{\square}\right)$ is defined on generators as

$$
\begin{aligned}
& {[(g, l)] \mapsto[g] \odot[l]=[g] \underline{\odot}[l], \quad g \in G, l \in L,} \\
& {\left[i_{G_{\square}}(s)\right] \mapsto[s] \odot 1=[s] \underline{\odot 1}, \quad s \in G_{\square},} \\
& {\left[i_{L_{\square}}(t)\right] \mapsto 1 \odot[t]=1 \underline{\varrho}[t], \quad t \in L_{\square},} \\
& {[\omega]=P(1 \mid 1)_{H} \mapsto P(1 \odot 1 \mid 1 \odot 1)_{H}, \quad \text { since } 1 \mapsto 1 \odot 1 \text {. }}
\end{aligned}
$$


It is straightforward to check that these formulas are compatible with the relations defining $A\left(G_{\square} \tilde{\times} L_{\square}\right)$, therefore they yield a quadratic pair algebra morphism. At the (0)-level this morphism is

$$
\mathbb{Z}_{\text {nil }}\left[(G \times L)_{+}\right]=\mathbb{Z}_{\text {nil }}\left[G_{+} \wedge L_{+}\right] \cong \mathbb{Z}_{\text {nil }}\left[G_{+}\right] \odot \mathbb{Z}_{\text {nil }}\left[L_{+}\right]
$$

the inverse of the isomorphism in Proposition 2.3.2. Using the computation of the homology of the "group-ring" of a sign group one can easily check that this quadratic pair algebra morphism is a weak equivalence, hence by the "five lemma" it is not only an isomorphism at the (0)-level, but also at the 1-level.

The following lemma gives a reinterpretation of sign group actions in terms of algebras and modules in the monoidal category qpm.

Lemma 3.3.8 Let $G_{\square}$ be a sign group and let $C$ be a 0 -good quadratic pair module. $A$ sign action of $G_{\square}$ on $C$ in the sense of Definition 3.3 .5 corresponds to a right $A\left(G_{\square}\right)$ module structure on $C$.

Proof With the notation in Definitions 3.3.5 and 3.3.6 the correspondence is given by the formulas, $g \in G, t \in G_{\square}$,

$$
\begin{aligned}
g^{*} x & =x \cdot[g], \\
\langle x, t\rangle & =x \cdot[t]+\left(\begin{array}{c}
\varepsilon(t) \\
2
\end{array}\right) P H(x) .
\end{aligned}
$$

The technical details of this proof are left to the reader.

In [10] we define a sign group action of $\operatorname{Sym}_{\square}(n)$ on $\Pi_{n, *} X$, hence combining Proposition 3.3.7 and Lemma 3.3.8 we readily obtain the following result.

Theorem 3.3.9 The sign group $\operatorname{Sym}_{\square}(n) \tilde{\times} \operatorname{Sym}_{\square}(m)$ acts on $\Pi_{n, *} X \odot \Pi_{m, *} Y$.

We will now consider the compatibility of the smash product operation in Theorem 3.2.1 with the sign group actions.

Theorem 3.3.10 The smash product morphism

$$
\wedge: \Pi_{n, *} X \odot \Pi_{m, *} Y \longrightarrow \Pi_{n+m, *}(X \wedge Y)
$$

in Theorem 3.2.1 is equivariant with respect to the action of $\operatorname{Sym}_{\square}(n) \tilde{\times} \operatorname{Sym}_{\square}(m)$ on $\Pi_{n, *} X \odot \Pi_{m, *} Y$ defined by Theorem 3.3.9 and the sign group morphism

$$
\operatorname{Sym}_{\square}(n) \tilde{\times} \operatorname{Sym}_{\square}(m) \longrightarrow \operatorname{Sym}_{\square}(n+n)
$$

in Proposition 3.3.4.

This theorem follows from Lemma 4.7.6 in Section 4.

Since the secondary homotopy groups $\Pi_{n, *} X$ have a canonical action of the sign group $\operatorname{Sym}_{\square}(n)$ we are led to consider the following category of symmetric sequences in qpm (this is similar to the treatment of symmetric spectra in [19]).

悬 Springer 
Definition 3.3.11 An object $X$ in the category $\mathbf{q p m}_{0}^{\text {Sym }}$ of symmetric sequences is a sequence of 0-good quadratic pair modules $X_{n}$ endowed with a sign group action of the symmetric track group $\operatorname{Sym}_{\square}(n), n \geq 0$. A morphism $f: X \rightarrow Y$ in $\mathbf{q p m}_{0}^{\operatorname{Sym}_{\square}}$ is a sequence of $\operatorname{Sym}_{\square}(n)$-equivariant morphisms $f_{n}: X_{n} \rightarrow Y_{n}$ in $\mathbf{q p m}_{0}$. The results in [10] show that secondary homotopy groups yield a functor

$$
\Pi_{*, *}: \text { Top }^{*} \longrightarrow \mathbf{q p m}_{0}^{\text {Sym }_{\square}} \text {. }
$$

The category $\mathbf{q p m}_{0}^{\text {Sym }} \square$ has a symmetric monoidal structure denoted by $\odot_{S_{y m}}$. The tensor product $X \odot_{\operatorname{sym}_{\square}} Y$ of two symmetric sequences $X, Y$ of 0 -good quadratic pair modules is characterized by the following universal property: for any symmetric sequence $Z$ of quadratic pair modules there is a natural bijection

$$
\operatorname{Hom}_{\mathbf{q p m}_{0}^{\text {Sym }} \square}\left(X \odot_{\operatorname{sym}_{\square}} Y, Z\right) \cong \prod_{p, q \in \mathbb{N}} \operatorname{Hom}_{\operatorname{Sym}_{\square}(p) \tilde{x} \operatorname{Sym}_{\square}(q)}\left(X_{p} \odot Y_{q}, Z_{p+q}\right) .
$$

Here $\operatorname{Hom}_{\mathrm{Sym}_{\square}(p) \tilde{\times} \operatorname{Sym}_{\square}(q)}$ denotes the set of morphisms in $\mathbf{q p m}$ which are equivariant with respect to the sign group morphism in Proposition 3.3.4. The explicit construction of $X \odot_{\operatorname{sym}_{\square}} Y$ is indicated in the appendix. The symmetry isomorphism

$$
X \odot_{\text {Sym }_{\square}} Y \cong Y \odot_{\text {Sym }_{\square}} X
$$

is induced by the morphisms in $\mathbf{q p m}$

$$
X_{p} \odot Y_{q} \cong Y_{q} \odot X_{p} \longrightarrow\left(Y \odot_{\mathrm{sym}_{\square}} X\right)_{q+p} \stackrel{\tau_{p, q}^{*}}{\longrightarrow}\left(Y \odot_{\mathrm{sym}_{\square}} X\right)_{p+q} .
$$

Here the first morphism is the symmetry isomorphism for $\odot$, the second one is induced by the universal property of $Y \odot_{\text {sym }} X$, and in the third morphism we use the sign group action of $\operatorname{Sym}_{\square}(p+q)$ and the shuffle permutation $\tau_{p, q} \in \operatorname{Sym}(p+q)$ in Eq. 18. The associativity isomorphism is defined by using the universal property of the 3 -fold tensor product, which is analogous to the 2 -fold case above. The unit element is $\overline{\mathbb{Z}}_{\text {nil }}$ concentrated in degree 0 .

Now Theorems 3.2.1, 3.3.10, 3.2.2 and 3.2.3 can be restated as follows.

Theorem 3.3.12 The smash product operator induces a natural morphism in the category $\mathbf{q p m}_{0}^{\mathrm{Sym}} \square$

$$
\wedge: \Pi_{*, *} X \odot_{\operatorname{sym}_{\square}} \Pi_{*, *} Y \longrightarrow \Pi_{*, *}(X \wedge Y)
$$

which is compatible with the associativity, commutativity and unit isomorphisms for the symmetric monoidal structures $\wedge$ and $\odot_{\mathrm{Sym}_{\square}}$ in $\mathbf{T} \mathbf{\mathbf { o p }}{ }^{*}$ and $\mathbf{q p m}_{0}^{\mathrm{Sym}_{\square}}$, respectively. Equivalently the functor

$$
\Pi_{*, *}: \text { Top }^{*} \longrightarrow \mathbf{q p m}_{0}^{\mathrm{Sym}_{\square}}
$$

given by secondary homotopy groups is lax symmetric monoidal.

\subsection{Secondary Whitehead Products}

The smash product may be used for the definition of Whitehead products in ordinary homotopy groups. In fact, any path connected space $X$ is homotopy equivalent to 
the classifying space of a topological group $G$ so that $\pi_{n} G=\pi_{n+1} X$. We consider the additive homotopy groups $\Pi_{n} G$, which satisfy

$$
\Pi_{n} G=\pi_{n} G \text { for } n \geq 1 \text {. }
$$

Using the smash product operator $\wedge$ in Eq. 15 for the functor $\Pi_{*}$ and the commutator map $c: G \wedge G \rightarrow G$ with $c(a \wedge b)=a^{-1} b^{-1} a b$ we obtain the composite

$$
[-,-]: \Pi_{*} G \otimes \Pi_{*} G \stackrel{\wedge}{\longrightarrow} \Pi_{*}(G \wedge G) \stackrel{\Pi_{*} c}{\longrightarrow} \Pi_{*} G,
$$

which corresponds to the Whitehead product in $\pi_{*} X$. It is well known that $\left(\Pi_{*} G,[-,-]\right)$ has the structure of a graded Lie algebra if $X$ is simply connected.

In a similar way we now define the secondary Whitehead product for the additive secondary homotopy groups $\Pi_{n, *} G$ by the composite

$$
[-,-]: \Pi_{*, *} G \otimes \Pi_{*, *} G \stackrel{\wedge}{\longrightarrow} \Pi_{*, *}(G \wedge G) \stackrel{\Pi_{*, *} c}{\longrightarrow} \Pi_{*, *} G .
$$

Marcum defines in [20] the partial Whitehead product of a map $\alpha$ and a track $F$ as in the following diagram

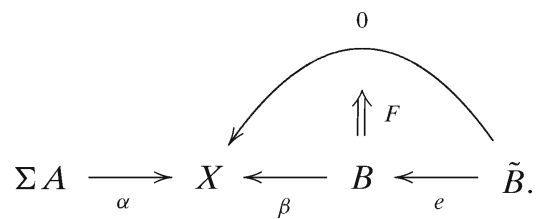

Marcum's partial Whitehead product lives in the group of homotopy classes [ $\Sigma A \wedge$ $\tilde{B}, X]$. It can be obtained from the secondary Whitehead product for additive secondary homotopy groups in case $A$ and $\tilde{B}$ are spheres.

We will explore this connection in a sequel of this paper where we shall discuss the algebraic properties of the structure $\left(\Pi_{*, *} G,[-,-]\right)$, which leads to the notion of a secondary Lie algebra. This should be compared with the notion of secondary Hopf algebra discussed in [3].

\subsection{Cup-one Products}

Let $n \geq m>1$ be even integers. The cup-one product operation

$$
\pi_{n} S^{m} \longrightarrow \pi_{2 n+1} S^{2 m}: \alpha \mapsto \alpha \smile_{1} \alpha=S q_{1}(\alpha)
$$

is defined in the following way, compare [18, 2.2.1]. Let $k$ be any positive integer and let $\tau_{k} \in \operatorname{Sym}(2 k)$ be the permutation exchanging the first and the second block of $k$ elements in $\{1, \ldots, 2 k\}$. If $k$ is even then sign $\tau_{k}=1$. We choose for any even integer 
$k>1$ a track $\hat{\tau}_{k}: \tau_{k} \Rightarrow 1_{S^{2 k}}$ in $\operatorname{Sym}_{\square}(2 k)$. Consider the following diagram in the track category Top* of pointed spaces where $a: S^{n} \rightarrow S^{m}$ represents $\alpha$.

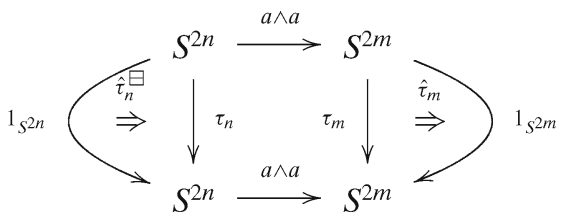

By pasting this diagram we obtain a self-track of $a \wedge a$

$$
\left(\hat{\tau}_{m}(a \wedge a)\right) \square\left((a \wedge a) \hat{\tau}_{n}^{\boxminus}\right): a \wedge a \Rightarrow a \wedge a .
$$

The set of self-tracks $a \wedge a \Rightarrow a \wedge a$ is the automorphism group of the map $a \wedge a$ in the track category Top*. The element $\alpha \smile_{1} \alpha \in \pi_{2 n+1} S^{2 m}$ is given by the track (29) via the well-known Barcus-Barratt-Rutter isomorphism

$$
\operatorname{Aut}(a \wedge a) \cong \pi_{2 n+1} S^{2 m},
$$

see [4], [21] and also [2, VI.3.12] and [5] for further details.

The following proposition yields a description of the cup-one product in terms of the structure of additive secondary homotopy groups.

Proposition 3.5.1 Let $n$ and $m$ be even positive integers. For $\alpha \in \Pi_{n} S^{m}$ we choose $a \in \Pi_{n, 0} S^{m}$ representing $\alpha$ and we define in $\Pi_{2 n, 1} S^{2 m}$

$$
S q_{1}(\alpha)=-\left\langle a \wedge a, \hat{\tau}_{n}\right\rangle+\left(\hat{\tau}_{m}\right)_{*}(a \wedge a)-P(H(a) \wedge T H(a)) .
$$

Then $\partial S q_{1}(\alpha)=0$ so that $S q_{1}(\alpha) \in h_{1} \Pi_{2 n, 1} S^{2 m}=\pi_{2 n+1} S^{2 m}$. Moreover, $S q_{1}(\alpha)=\alpha \smile_{1} \alpha$.

Proof The track $\hat{\tau}_{m}: \tau_{m} \Rightarrow 1$ induces a track $\left(\hat{\tau}_{m}\right)_{*}:\left(\tau_{m}\right)_{*} \Rightarrow 1$ in $\mathbf{q p m}$ satisfying

$$
\partial\left(\hat{\tau}_{m}\right)_{*}(a \wedge a)=-\left(\tau_{m}\right)_{*}(a \wedge a)+a \wedge a .
$$

By the symmetric action we have the element $\left\langle a \wedge a, \hat{\tau}_{n}\right\rangle \in \Pi_{2 n, 1} S^{2 m}$ satisfying

$$
-\partial\left\langle a \wedge a, \hat{\tau}_{n}\right\rangle=-a \wedge a+\tau_{n}^{*}(a \wedge a) .
$$

Hence we get

$$
\partial S q_{1}(\alpha)=-a \wedge a+\left(\tau_{n}\right)^{*}(a \wedge a)-\left(\tau_{m}\right)_{*}(a \wedge a)+a \wedge a-\partial P(H(a) \wedge T H(a))
$$

where $\left(\tau_{n}\right)^{*}(a \wedge a)=\left(\tau_{m}\right)_{*}(a \wedge a)$ and $a \wedge a-a \wedge a=\partial P(H(a) \wedge T H(a))$. This shows $\partial S q_{1}(\alpha)=0$. Using the definition of secondary homotopy groups as track functors in [9] and the symmetric actions in [10] we see that $S q_{1}(\alpha)$ coincides with the track definition of $\alpha \smile_{1} \alpha$.

Theorem 3.5.2 Let $n$ and $m$ be even positive integers and $\alpha, \beta \in \pi_{n} S^{m}$. Then

$$
S q_{1}(\alpha \mid \beta)=S q_{1}(\alpha+\beta)-S q_{1}(\alpha)-S q_{1}(\beta)=\left(\frac{n-m}{2}+1\right)(\alpha \wedge \beta)\left(\Sigma^{2 n-3} \eta\right) .
$$

This result is stated in [6], but a proof did not appear in the literature. 
Proof of Theorem 3.5.2 We choose representatives $a, b \in \Pi_{n, 0} S^{m}$ of $\alpha$, $\beta$ with

$$
H(a)=0=H(b) .
$$

Then we have

$$
a \wedge b=a \wedge b
$$

and we get

$$
\begin{aligned}
(a+b) \wedge(a+b) & =a \wedge(a+b)+b \wedge(a+b) \\
& =a \wedge a+a \wedge b+b \wedge a+b \wedge b \\
& =x+u+y, \\
(a+b) \wedge(a+b) & =(a+b) \wedge a+(a+b) \wedge b \\
& =a \wedge a+b \wedge a+a \wedge b+b \wedge b \\
& =x+v+y .
\end{aligned}
$$

Here we set $x=a \wedge a, y=b \wedge b$ and

$$
\begin{aligned}
& u=a \wedge b+b \wedge a, \\
& v=b \wedge a+a \wedge b,
\end{aligned}
$$

so that

$$
u=v+\partial P(a \wedge b \mid b \wedge a)_{H} .
$$

Now the formula for $S q_{1}(\alpha)$ yields:

$$
\begin{aligned}
& S q_{1}(\alpha)=-\left\langle a \wedge a, \hat{\tau}_{n}\right\rangle+\left(\hat{\tau}_{m}\right)_{*}(a \wedge a), \\
& S q_{1}(\beta)=-\left\langle b \wedge b, \hat{\tau}_{n}\right\rangle+\left(\hat{\tau}_{m}\right)_{*}(b \wedge b) .
\end{aligned}
$$

Moreover for $\gamma=\alpha+\beta$ represented by $c=a+b$ we have

$$
S q_{1}(\gamma)=-\left\langle c \wedge c, \hat{\tau}_{n}\right\rangle+\left(\hat{\tau}_{m}\right)_{*}(c \wedge c)-P(H(c) \wedge T H(c)) .
$$

The summands of $S q_{1}(\gamma)$ satisfy the formulas:

$$
\begin{aligned}
\left\langle c \wedge c, \hat{\tau}_{n}\right\rangle= & \left\langle x+u+y, \hat{\tau}_{n}\right\rangle \\
= & \left\langle x, \hat{\tau}_{n}\right\rangle^{\tau_{n}^{*}(u+y)}+\left\langle u, \hat{\tau}_{n}\right\rangle^{\tau_{n}^{*}(y)}+\left\langle y, \hat{\tau}_{n}\right\rangle, \\
\left(\hat{\tau}_{m}\right)_{*}(c \wedge c)= & \left(\hat{\tau}_{m}\right)_{*}(x+v+y) \\
= & \left(\hat{\tau}_{m}\right)_{*}(x)^{\left(\tau_{m}\right)_{*}(v+y)} \\
& +\left(\hat{\tau}_{m}\right)_{*}(v)^{\left(\tau_{m}\right)_{*}(y)}+\left(\hat{\tau}_{m}\right)_{*}(y) .
\end{aligned}
$$

Here we have

$$
\begin{aligned}
\left(\tau_{n}\right)^{*}(a \wedge b) & =\left(\tau_{m}\right)_{*}(b \wedge a) \\
& =\left(\tau_{m}\right)_{*}(b \wedge a), \\
\left(\tau_{n}\right)^{*}(u) & =\left(\tau_{m}\right)_{*}(v) .
\end{aligned}
$$


Hence we get

$$
\begin{aligned}
S q_{1}(\gamma)= & -\left\langle y, \hat{\tau}_{n}\right\rangle-\left\langle u, \hat{\tau}_{n}\right\rangle^{\tau_{n}^{*}(y)} \\
& +\left(-\left\langle x, \hat{\tau}_{n}\right\rangle+\left(\hat{\tau}_{m}\right)_{*}(x)\right)^{\left(\tau_{m}\right)_{*}(v+y)} \\
& +\left(\hat{\tau}_{m}\right)_{*}(v)^{\left(\tau_{m}\right)_{*}(y)}+\left(\hat{\tau}_{m}\right)_{*}(y)-P(H(c) \wedge T H(c)) .
\end{aligned}
$$

Since the action on Ker $\partial$ is trivial and since the image of $P$ and Ker $\partial$ are both central we thus get

$$
\begin{aligned}
S q_{1}(\gamma)-S q_{1}(\beta)= & -\left\langle u, \hat{\tau}_{n}\right\rangle^{\tau_{n}^{*}(y)}+S q_{1}(\alpha)+\left(\hat{\tau}_{m}\right)_{*}(v)^{\left(\tau_{m}\right)_{*}(y)} \\
& -P(H(c) \wedge T H(c)) .
\end{aligned}
$$

Therefore we have

$$
\begin{aligned}
S q_{1}(\alpha \mid \beta) & =-\left\langle u, \hat{\tau}_{n}\right\rangle^{\tau_{n}^{*}(y)}+\left(\hat{\tau}_{m}\right)_{*}(v)^{\left(\tau_{m}\right)_{*}(y)}-P(H(c) \wedge T H(c)) \\
& =\left(-\left\langle u, \hat{\tau}_{n}\right\rangle+\left(\hat{\tau}_{m}\right)_{*}(v)\right)^{\left(\tau_{m}\right)_{*}(y)}+P(a \wedge b \mid b \wedge a)_{H}
\end{aligned}
$$

since $\partial\left(-\left\langle u, \hat{\tau}_{n}\right\rangle+\left(\hat{\tau}_{m}\right)_{*}(v)\right)$ is a commutator, and hence in the image of $\partial P$. Here we have

$$
\begin{aligned}
\left\langle u, \hat{\tau}_{n}\right\rangle= & \left\langle a \wedge b, \hat{\tau}_{n}\right\rangle+\left\langle b \wedge a, \hat{\tau}_{n}\right\rangle+P\left(-\tau_{n}^{*}(a \wedge b)+a \wedge b \mid \tau_{n}^{*}(b \wedge a)\right)_{H}, \\
\left(\hat{\tau}_{m}\right)_{*}(v)= & \left(\hat{\tau}_{m}\right)_{*}(b \wedge a)+\left(\hat{\tau}_{m}\right)_{*}(a \wedge b) \\
& +P\left(-\left(\tau_{m}\right)_{*}(b \wedge a)+b \wedge a \mid\left(\tau_{m}\right)_{*}(a \wedge b)\right)_{H} .
\end{aligned}
$$

Hence we obtain

$$
\begin{aligned}
S q_{1}(a \mid b) & =-\left\langle b \wedge a, \hat{\tau}_{n}\right\rangle-\left\langle a \wedge b, \hat{\tau}_{n}\right\rangle+\left(\hat{\tau}_{m}\right)_{*}(b \wedge a)+\left(\hat{\tau}_{m}\right)_{*}(a \wedge b)+(\mathrm{c}), \\
(\mathrm{c}) & =P\left(b \wedge a-a \wedge b \mid\left(\hat{\tau}_{m}\right)_{*}(a \wedge b)\right)+P(a \wedge b \mid b \wedge a)_{H} .
\end{aligned}
$$

Now we consider the following formulas with $\partial(a)=0=\partial(b)$.

$$
\begin{aligned}
(\mathrm{a}) & =\left\langle a \wedge b, \hat{\tau}_{n} \hat{\tau}_{n}\right\rangle \\
& =\left\langle\tau_{n}^{*}(a \wedge b), \hat{\tau}_{n}\right\rangle+\left\langle a \wedge b, \hat{\tau}_{n}\right\rangle, \\
(\mathrm{b}) & =\left(\hat{\tau}_{m} \hat{\tau}_{m}\right)_{*}(a \wedge b) \\
& =\left(\hat{\tau}_{m}\right)_{*}\left(\left(\tau_{m}\right)_{*}(a \wedge b)\right)+\left(\hat{\tau}_{m}\right)_{*}(a \wedge b) .
\end{aligned}
$$

For (b) we use that $\Pi_{2 n, *}$ is a track functor and

$$
\begin{aligned}
\hat{\tau}_{m} \hat{\tau}_{m} & =\hat{\tau}_{m} \square\left(\tau_{m} \hat{\tau}_{m}\right) \\
& =\hat{\tau}_{m} \square\left(\hat{\tau}_{m} \tau_{m}\right) .
\end{aligned}
$$


Now we have the following equations.

$$
\begin{aligned}
\left\langle a \wedge b, \hat{\tau}_{n}\right\rangle+\left\langle b \wedge a, \hat{\tau}_{n}\right\rangle & =(\mathrm{a})-\left\langle\tau_{n}^{*}(a \wedge b), \hat{\tau}_{n}\right\rangle+\left\langle b \wedge a, \hat{\tau}_{n}\right\rangle \\
& =(\mathrm{a})+\left\langle-\left(\tau_{m}\right)_{*}(b \wedge a)+b \wedge a, \hat{\tau}_{n}\right\rangle-(\mathrm{d}) \\
& =(\mathrm{a})+\left\langle\partial\left(\hat{\tau}_{m}\right)_{*}(b \wedge a), \hat{\tau}_{n}\right\rangle-(\mathrm{d}) \\
& =(\mathrm{a})-\tau_{n}^{*}\left(\hat{\tau}_{m}\right)_{*}(b \wedge a)+\left(\hat{\tau}_{m}\right)_{*}(b \wedge a)-(\mathrm{d}) \\
& =(\mathrm{a})-\left(\hat{\tau}_{m}\right)_{*} \tau_{n}^{*}(b \wedge a)+\left(\hat{\tau}_{m}\right)_{*}(b \wedge a)-(\mathrm{d}) \\
& =(\mathrm{a})-\left(\hat{\tau}_{m}\right)_{*}\left(\tau_{m}\right)_{*}(a \wedge b)+\left(\hat{\tau}_{m}\right)_{*}(b \wedge a)-(\mathrm{d}) .
\end{aligned}
$$

Here (d) is given by the following formula.

$$
\begin{aligned}
(\mathrm{d})= & \left.-P\left(\tau_{n}^{*}\left(\tau_{m}\right)_{*}(b \wedge a)-\left(\tau_{m}\right)_{*}(b \wedge a)\right) \mid \tau_{n}^{*}\left(\tau_{m}\right)_{*}(b \wedge a)\right)_{H} \\
& \left.+P\left(\tau_{n}^{*}\left(\tau_{m}\right)_{*}(b \wedge a)-\left(\tau_{m}\right)_{*}(b \wedge a)\right) \mid \tau_{n}^{*}(b \wedge a)\right)_{H} \\
= & -P\left(a \wedge b-\left(\tau_{m}\right)_{*}(b \wedge a) \mid a \wedge b\right)_{H} \\
& +P\left(a \wedge b-\left(\tau_{m}\right)_{*}(b \wedge a) \mid \tau_{n}^{*}(b \wedge a)\right)_{H} \\
= & -P(a \wedge b \mid a \wedge b)_{H}+P\left(\left(\tau_{m}\right)_{*}(b \wedge a) \mid a \wedge b\right)_{H} \\
& +P\left(a \wedge b \mid\left(\tau_{m}\right)_{*}(b \wedge a)\right)_{H}-P\left(\left(\tau_{m}\right)_{*}(b \wedge a) \mid\left(\tau_{m}\right)_{*}(a \wedge b)\right)_{H} .
\end{aligned}
$$

On the other hand we get

$$
\begin{aligned}
\left(\hat{\tau}_{m}\right)_{*}(b \wedge a)+\left(\hat{\tau}_{m}\right)_{*}(a \wedge b)= & \left(\hat{\tau}_{m}\right)_{*}(b \wedge a) \\
& -\left(\hat{\tau}_{m}\right)_{*}\left(\left(\tau_{m}\right)_{*}(a \wedge b)\right)+(\mathrm{b}) .
\end{aligned}
$$

Now we get

$$
\begin{aligned}
S q_{1}(\alpha \mid \beta)= & -(\mathrm{a})-(\mathrm{d})-\left(\hat{\tau}_{m}\right)_{*}(b \wedge a)+\left(\hat{\tau}_{m}\right)_{*}\left(\left(\tau_{m}\right)_{*}(a \wedge b)\right) \\
& +\left(\hat{\tau}_{m}\right)_{*}(b \wedge a)-\left(\hat{\tau}_{m}\right)_{*}\left(\left(\tau_{m}\right)_{*}(a \wedge b)\right)+(\mathrm{b})+(\mathrm{c}) \\
= & -(\mathrm{a})+(\mathrm{d})+(\mathrm{e})+(\mathrm{b})+(\mathrm{c})
\end{aligned}
$$

where (e) is the commutator:

$$
\begin{aligned}
(\mathrm{e})= & P\left(\left(\hat{\tau}_{m}\right)_{*}(b \wedge a) \mid-\left(\hat{\tau}_{m}\right)_{*}\left(\left(\tau_{m}\right)_{*}(a \wedge b)\right)_{H \partial}\right. \\
= & P\left(-\left(\tau_{m}\right)_{*}(b \wedge a)+b \wedge a \mid\left(\tau_{m} \tau_{m}\right)_{*}(a \wedge b)\right. \\
& \left.-\left(\tau_{m}\right)_{*}(a \wedge b)\right)_{H} \\
= & -P\left(\left(\tau_{m}\right)_{*}(b \wedge a) \mid a \wedge b\right)_{H}+P(b \wedge a \mid a \wedge b)_{H} \\
& +P\left(\left(\tau_{m}\right)_{*}(b \wedge a) \mid\left(\tau_{m}\right)_{*}(a \wedge b)\right)_{H} \\
& -P\left(b \wedge a \mid\left(\tau_{m}\right)_{*}(a \wedge b)\right)_{H} .
\end{aligned}
$$




$$
\begin{aligned}
(\mathrm{d})+(\mathrm{e})+(\mathrm{c})= & -P(a \wedge b \mid a \wedge b)_{H}+P\left(\left(\tau_{m}\right)_{*}(b \wedge a) \mid a \wedge b\right)_{H} \\
& +P\left(a \wedge b \mid\left(\tau_{m}\right)_{*}(a \wedge b)\right)_{H} \\
& -P\left(\left(\tau_{m}\right)_{*}(b \wedge a) \mid\left(\tau_{m}\right)_{*}(a \wedge b)\right)_{H} \\
& -P\left(\left(\tau_{m}\right)_{*}(b \wedge a) \mid a \wedge b\right)_{H}+P(b \wedge a \mid a \wedge b)_{H} \\
& +P\left(\left(\tau_{m}\right)_{*}(b \wedge a) \mid\left(\tau_{m}\right)_{*}(a \wedge b)\right)_{H} \\
& -P\left(b \wedge a \mid\left(\tau_{m}\right)_{*}(a \wedge b)\right)_{H} \\
& +P\left(b \wedge a \mid\left(\tau_{m}\right)_{*}(a \wedge b)\right)_{H}-P\left(a \wedge b \mid\left(\tau_{m}\right)_{*}(a \wedge b)\right)_{H} \\
& +P(a \wedge b \mid b \wedge a)_{H} \\
= & -P(a \wedge b \mid a \wedge b)_{H} .
\end{aligned}
$$

Hence we get

$$
S q_{1}(\alpha \mid \beta)=-(\mathrm{a})+(\mathrm{b})-P(a \wedge b \mid a \wedge b)_{H},
$$

and this implies the result by use of Proposition 3.1.2.

\subsection{Toda Brackets}

For a pointed space $X$ we use the suspension $\Sigma X=S^{1} \wedge X$ and the E-suspension $E X=X \wedge S^{1}$. Here $\Sigma$ and $E$ are isomorphic endofunctors of Top*. The $E$ suspension is for example used by Toda in his book [22].

Definition 3.6.1 Let $n \geq k \geq 0$ and consider morphisms in Top* $/ \simeq$

$$
Z \stackrel{\alpha}{\leftarrow} E^{k} Y \stackrel{E^{k} \beta}{\leftarrow} E^{k} X, \quad Y \stackrel{\beta}{\leftarrow} X \stackrel{\gamma}{\leftarrow} S^{n-k}
$$

with $\alpha\left(E^{k} \beta\right)=0$ and $\beta \gamma=0$. Then the Toda bracket

$$
\left\{\alpha, E^{k} \beta, E^{k} \gamma\right\}_{k} \subset \pi_{n+1} Z
$$

is the subset of all elements in $\pi_{n+1} Z$ obtained by pasting tracks as in the diagram

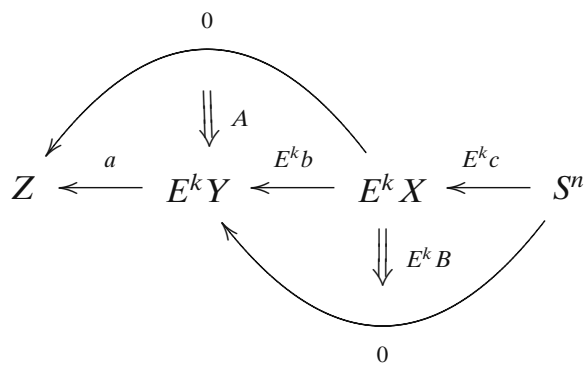

where $a, b, c$ represent $\alpha, \beta, \gamma$ and $B: b c \Rightarrow 0$ and $A: 0 \Rightarrow a\left(E^{k} b\right)$. 
Let $\iota_{k} \in \pi_{k}\left(S^{k}\right)$ be the element represented by the identity of $S^{k}, k \geq 0$. Moreover, let

$$
\iota_{k, 0} \in \Omega^{k} S^{k} \subset \Pi_{k, 0}\left(S^{k}\right)=\left\langle\Omega^{k} S^{k}\right\rangle_{n i l}
$$

be given by the identity of $S^{k}$. This element yields a quadratic pair module morphism

$$
\iota_{k, 0}: \overline{\mathbb{Z}}_{n i l} \longrightarrow \Pi_{k, *} S^{k}
$$

We define the morphisms $\bar{E}^{k}$ in $\mathbf{q p m}, n \geq k$,

$$
\bar{E}^{k}: \Pi_{n-k, *} X \cong \Pi_{n-k, *} X \odot \overline{\mathbb{Z}}_{n i l} \stackrel{1 \otimes l_{k, 0}}{\rightarrow} \Pi_{n-k, *} X \odot \Pi_{k, *} S^{k} \stackrel{\wedge}{\rightarrow} \Pi_{n, *}\left(E^{k} X\right)
$$

Let $\alpha, \beta, \gamma$ be given as in Definition 3.6.1 with $\alpha\left(E^{k} \beta\right)=0$ and $\beta \gamma=0$. We choose maps $a, b$ representing $\alpha, \beta$ and we choose a track $A: 0 \Rightarrow a\left(E^{k} b\right)$ as in Definition 3.6.1. Moreover, let

$$
\bar{c} \in \Pi_{n-k, 0} X
$$

be an element representing $\gamma \in \pi_{n-k} X$ with $n-k \geq 2$ and let

$$
\bar{B} \in \Pi_{n-k, 1} Y
$$

be an element with $\partial \bar{B}=b_{*}(\bar{c}) \in \Pi_{n-k, 0} Y$. Then $\bar{E}^{k} \bar{B} \in \Pi_{n, 1} E^{k} Y$ satisfies

$$
\partial \bar{E}^{k} \bar{B}=\partial\left(\bar{B} \wedge \imath_{k, 0}\right)=(\partial \bar{B}) \wedge \imath_{k, 0}=b_{*}(\bar{c}) \wedge l_{k, 0}=\left(E^{k} b\right)_{*}\left(\bar{c} \wedge \imath_{k, 0}\right) .
$$

Moreover, the track $A$ induces a track in $\mathbf{q p m}$ which is given by a map

$$
A_{*}: \Pi_{n, 0} E^{k} X \longrightarrow \Pi_{n, 1} Z
$$

with $\partial A_{*}(x)=\left(a\left(E^{k} b\right)\right)_{*}(x)$. Therefore the element

$$
t=a_{*}\left(E^{k} \bar{B}\right)-A_{*}\left(\bar{c} \wedge l_{k, 0}\right) \in \Pi_{n, 1} Z
$$

satisfies $\partial(t)=0$ and hence $t$ is an element in $h_{1} \Pi_{n, *} Z$. Recall from Eq. 23 that $h_{1} \Pi_{n, *} Z$ is naturally isomorphic to $\pi_{n+1} Z$ for $n \geq 3$ and to $\pi_{3} Z /\left[\pi_{2} Z, \pi_{2} Z\right.$ ] for $n=2$, where $[-,-]$ is the Whitehead product.

Lemma 3.6.2 For $n \geq 3, a_{*} \bar{E}^{k}(\bar{B})-A_{*}\left(\bar{c} \wedge \imath_{k, 0}\right) \in\left\{\alpha, E^{k} \beta, E^{k} \gamma\right\}_{k}$. Moreover, all elements in $\iota\left\{\alpha, E^{k} \beta, E^{k} \gamma\right\}_{k}$ can be obtained in this way. The same equality holds for $n=2 \bmod \left[\pi_{2} Z, \pi_{2} Z\right]$, the image of the Whitehead product.

Proposition 3.6.3 Let $Y$ be a pointed space and let $r \in \mathbb{Z}$ and $\beta \in \pi_{n-1} Y$ with $r \beta=0$. In the group of homotopy classes $[E Y, E Y]$ let $r 1_{E Y}$ be the $r$-fold sum of the identity $1_{E Y}$. Then the Toda bracket $\left\{r 1_{E Y}, E \beta, r l_{n}\right\}_{1} \subset \pi_{n+1} E Y$ is defined and for $n \geq 3$

$$
\left\{r 1_{E Y}, E \beta, r l_{n}\right\}_{1} \ni \begin{cases}0, & \text { if } r \text { is odd, } \\ \frac{r}{2}(E \beta)\left(\Sigma^{n-2} \eta\right) & \text { if } r \text { is even. }\end{cases}
$$

Here $\eta: S^{3} \rightarrow S^{2}$ is the Hopf map. For $n=2$ the same formula holds in the quotient $\pi_{3} E Y /\left[\pi_{2} E Y, \pi_{2} E Y\right]$ where $[-,-]$ is the Whitehead product.

In $[22,3.7]$ Toda proves this result in case $Y$ is a sphere. Toda's proof uses different methods relying on the assumption that $Y$ is a sphere. 
Proof of Proposition 3.6.3 Let $(\cdot)^{r}: S^{1} \rightarrow S^{1}$ be the degree $r$ map $z \mapsto z^{r}$ so that $r 1_{E Y}$ is represented by $a=Y \wedge(\cdot)^{r}$. Let $b$ be a map representing $\beta$. We choose $\bar{c} \in \Pi_{n-1,0} S^{n-1}$ representing $r l_{n}$ by $\bar{c}=r l_{n-1,0}$ and we choose

$$
\bar{B} \in \Pi_{n-1,1} Y
$$

with $\partial \bar{B}=b_{*}(\bar{c})=r b_{*}\left(l_{n-1,0}\right)$. Then we get as in Eq. 31

$$
\partial \bar{E}(\bar{B})=(E b)_{*}\left(\bar{c} \wedge \iota_{1,0}\right)
$$

where $\bar{c} \wedge l_{1,0}=r\left(l_{n-1,0} \wedge l_{1,0}\right)=r l_{n, 0}$. Now we choose $A: 0 \Rightarrow a(E b)$ in such a way that the induced track $A_{*}$ satisfies

$$
A_{*}\left(l_{n, 0}\right)=\bar{E}(\bar{B}) .
$$

In fact, the boundary of $A_{*}\left(l_{n, 0}\right)$ is

$$
\partial A_{*}\left(l_{n, 0}\right)=(a(E b))_{*}\left(l_{n, 0}\right)=\partial \bar{E}(\bar{B})
$$

where $a(E b)=\left(Y \wedge(\cdot)^{r}\right)\left(b \wedge S^{1}\right)=b \wedge(\cdot)^{r}=(E b)\left(\Sigma^{n-1}(\cdot)^{r}\right)$ and $\left(\Sigma^{n-1}(\cdot)^{r}\right)_{*}\left(l_{n, 0}\right)=$ $r^{*} l_{n, 0}=r l_{n, 0}$. since $H\left(i_{n, 0}\right)=0$.

Now we can compute the element in Lemma 3.6.2

$$
t=a_{*} \bar{E}(\bar{B})-A_{*}\left(\bar{c} \wedge \iota_{1,0}\right) \in\left\{r 1_{E Y}, E \beta, r l_{n}\right\}_{1} .
$$

Here $A$ is a track $f_{0} \Rightarrow g_{0}$ with $f_{0}=0$ so that

$$
\begin{aligned}
A_{*}\left(\bar{c} \wedge l_{1,0}\right) & =A_{*}\left(r l_{n, 0}\right) \\
& =r A_{*}\left(l_{n, 0}\right) \\
& =r \bar{E} \bar{B} .
\end{aligned}
$$

On the other hand we get

$$
\begin{aligned}
a_{*}(\bar{E} \bar{B}) & =\left(Y \wedge(\cdot)^{r}\right)_{*}\left(\bar{B} \wedge l_{1,0}\right) \\
& =\bar{B} \wedge\left(\left((\cdot)^{r}\right)_{*}\left(l_{1,0}\right)\right) \\
& =\bar{B} \wedge\left(r^{*} l_{1,0}\right) \\
& =\bar{B} \wedge\left(r l_{1,0}\right) \\
& =r\left(\bar{B} \wedge l_{1,0}\right)+t \\
& =r \bar{E}(\bar{B})+t
\end{aligned}
$$

where

$$
\begin{aligned}
t & =\left(\begin{array}{l}
r \\
2
\end{array}\right) P\left(H \partial(\bar{B}) \wedge\left(l_{1,0} \mid l_{1,0}\right)_{H}\right) \\
& =\left(\begin{array}{l}
r \\
2
\end{array}\right) P\left(H\left(r b_{*}\left(l_{n-1,0}\right)\right) \wedge\left(l_{1,0} \mid l_{1,0}\right)_{H}\right) \\
& =\left(\begin{array}{l}
r \\
2
\end{array}\right)^{2} P\left(\left(b_{*}\left(l_{n-1,0}\right) \mid b_{*}\left(l_{n-1,0}\right)\right)_{H} \wedge\left(l_{1,0} \mid l_{1,0}\right)_{H}\right) \\
& =\left(\begin{array}{l}
r \\
2
\end{array}\right)^{2} P\left(b_{*}\left(l_{n-1,0}\right) \wedge l_{1,0} \mid b_{*}\left(l_{n-1,0}\right) \wedge l_{1,0}\right)_{H} .
\end{aligned}
$$


Therefore $t$ represents $\left(\begin{array}{l}r \\ 2\end{array}\right)^{2}(E \beta)\left(\Sigma^{n-2} \eta\right)$. If $r$ is odd we see that $t=0$ since $\beta$ has odd order and $\Sigma \eta$ has even order.

\section{The Construction of the Smash Product for Secondary Homotopy Groups}

In this part we define the smash product operator for secondary homotopy groups and we prove the results described in Section 3. A crucial step for this definition will be the construction of canonical tracks

$$
\Sigma\left(f \underline{\#)} \stackrel{\mathbb{H}_{\#}^{\#}}{\Rightarrow} f \wedge g \stackrel{\mathbb{H}^{\#}}{\Leftarrow} \Sigma(f \# g),\right.
$$

termed the exterior tracks, connecting the exterior cup-products and the smash product of two maps. Then we use $\mathbb{H}^{\#}$ and $\mathbb{H}^{\#}$ for the definition of the smash product operator on the (1)-level. Some of the algebraic properties of the smash product are then derived from formulas concerning the Hopf invariant of the track $\left(\mathbb{H}^{\#}\right)^{\boxminus} \square \mathbb{H}^{\#}$, and of some other tracks between suspensions which are built out of the exterior tracks.

\subsection{Exterior Cup-products for Higher Suspensions and Tracks}

We begin this section by stating the basic properties of the exterior cup-product operations.

Lemma 4.1.1 We have the following formulas for suspensions

(1) $f \#\left(\Sigma g^{\prime}\right)=f \wedge g^{\prime}=f \underline{\#}\left(\Sigma g^{\prime}\right)$,

(2) $\left(\Sigma f^{\prime}\right) \# g=\left(\begin{array}{ll}1 & 2\end{array}\right)\left(f^{\prime} \wedge g\right)(12)=\left(\Sigma f^{\prime}\right) \# g$,

coproducts

(3) $\left(f_{1}, f_{2}\right) \# g=\left(f_{1} \# g, f_{2} \# g\right)$,

(4) $\left(f_{1}, f_{2}\right) \# g=\left(f_{1} \# g, f_{2} \# g\right)$,

(5) $f \#\left(g_{1}, g_{2}\right)=\left(f \# g_{1}, f \# g_{2}\right)$,

(6) $f \underline{\#}\left(g_{1}, g_{2}\right)=\left(f \underline{\#} g_{1}, f \# g_{2}\right)$,

and compositions

(7) $\left(f_{1} f_{2}\right) \#\left(g_{1}\left(\Sigma g_{2}^{\prime}\right)\right)=\left(f_{1} \# g_{1}\right)\left(f_{2} \#\left(\Sigma g_{2}^{\prime}\right)\right)$,

(8) $\left(\left(\Sigma f_{1}^{\prime}\right) f_{2}\right) \#\left(g_{1} g_{2}\right)=\left(\left(\Sigma f_{1}^{\prime}\right) \# g_{1}\right)\left(f_{2} \# g_{2}\right)$,

(9) $\left(f_{1}\left(\Sigma f_{2}^{\prime}\right)\right) \#\left(g_{1} g_{2}\right)=\left(f_{1} \# g_{1}\right)\left(\left(\Sigma f_{2}^{\prime}\right) \# g_{2}\right)$,

(10) $\left.\quad\left(f_{1} f_{2}\right) \underline{\#(}\left(\Sigma g_{1}^{\prime}\right) g_{2}\right)=\left(f_{1} \#\left(\Sigma g_{1}^{\prime}\right)\right)\left(f_{2} \# g_{2}\right)$.

The exterior cup-products are associative

(11) $f \#(g \# h)=(f \# g) \# h$,

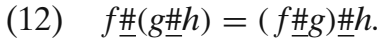

The proof of this lemma is straightforward.

In order to define the exterior cup-products

$$
f \# g, f \# g: \Sigma^{n+m-1} A \wedge Y \rightarrow \Sigma^{n+m-1} B \wedge Y,
$$


of maps between higher suspensions $f: \Sigma^{n} A \rightarrow \Sigma^{n} B, g: \Sigma^{m} X \rightarrow \Sigma^{m} Y$ we take the first spherical coordinates to the end of the smash product

$$
\begin{aligned}
& \bar{f}: S^{1} \wedge A \wedge S^{n-1} \cong S^{n-1} \wedge S^{1} \wedge A \stackrel{f}{\longrightarrow} S^{n-1} \wedge S^{1} \wedge B \cong S^{1} \wedge B \wedge S^{n-1} \\
& \bar{g}: S^{1} \wedge X \wedge S^{m-1} \cong S^{m-1} \wedge S^{1} \wedge X \stackrel{g}{\longrightarrow} S^{m-1} \wedge S^{1} \wedge Y \cong S^{1} \wedge Y \wedge S^{m-1}
\end{aligned}
$$

Then we perform the usual exterior cup product on these maps, and we recollect the permuted spherical coordinates at the beginning of the smash product in an ordered way,

$$
\begin{aligned}
f \# g: S^{n-1} \wedge S^{m-1} \wedge S^{1} \wedge A \wedge X & \cong S^{1} \wedge A \wedge S^{n-1} \wedge X \wedge S^{m-1} \\
& \stackrel{\bar{f} \# \bar{g}}{\longrightarrow} S^{1} \wedge B \wedge S^{n-1} \wedge Y \wedge S^{m-1} \\
& \cong S^{n-1} \wedge S^{m-1} \wedge S^{1} \wedge B \wedge Y .
\end{aligned}
$$

The same for \#. These exterior cup-products generalize the classical ones in the following sense. If $f=\Sigma^{n-1} f^{\prime}$ and $g=\Sigma^{m-1} g^{\prime}$ for $f: \Sigma A \rightarrow \Sigma B$ and $g: \Sigma X \rightarrow \Sigma Y$ then

$$
\begin{aligned}
& \left(\Sigma^{n-1} f^{\prime}\right) \#\left(\Sigma^{m-1} g^{\prime}\right)=\Sigma^{n+m-1}\left(f^{\prime} \# g^{\prime}\right), \\
& \left(\Sigma^{n-1} f^{\prime}\right) \#\left(\Sigma^{m-1} g^{\prime}\right)=\Sigma^{n+m-1}\left(f^{\prime} \# g^{\prime}\right) \text {. }
\end{aligned}
$$

The properties of the classical exterior cup-products in Lemma 4.1.1 can be accordingly restated for the exterior cup-product of maps between higher suspensions.

Let $F: f \Rightarrow g, G: h \Rightarrow k$ be now tracks between maps $f, g: \Sigma^{n} A \rightarrow \Sigma^{n} B$, $h, k: \Sigma^{m} X \rightarrow \Sigma^{m} Y$. The exterior products of a track with a map

$$
\begin{aligned}
& F \# h: f \# h \Rightarrow g \# h, \\
& F \# h: f \underline{\# h} \Rightarrow g \underline{\# h}, \\
& f \# G: f \# h \Rightarrow f \# k, \\
& f_{\#}^{\#} G: f \underline{\# h} \Rightarrow f \underline{\# k},
\end{aligned}
$$

are defined by exchanging the interval $I_{+}$with the spherical coordinates and using the exterior cup-products of maps between higher suspensions as defined above. For example the track $F \# h$ is represented by the homotopy

$$
I_{+} \wedge S^{n+m-1} \wedge A \wedge X \cong S^{n+m-1} \wedge I_{+} \wedge A \wedge X \stackrel{\tilde{F} \# h}{\longrightarrow} S^{n+m-1} \wedge B \wedge Y,
$$

where

$$
\tilde{F}: S^{n-1} \wedge I_{+} \wedge A \cong I_{+} \wedge S^{n-1} \wedge A \stackrel{F}{\longrightarrow} S^{n-1} \wedge B
$$

is defined from a homotopy $F$ representing the corresponding track. Now one can define the exterior products of two tracks as the vertical composition

$$
\begin{aligned}
& F \# G=(g \# G) \square(F \# h)=(F \# k) \square(f \# G),
\end{aligned}
$$

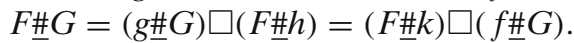

The reader may notice in these equations the same interchange behaviour as in the definition of the smash product of tracks, see Section 2.3. This will often be relevant 
for computations. It follows from the fact that the operation $f \# g$ is continuous in both $f$ and $g$, so given two continuous families of maps (paths in mapping spaces) $\left\{f_{s}\right\}_{s \in[0,1]},\left\{g_{t}\right\}_{t \in[0,1]}$ there is essentially a single way of reindexing $\left\{f_{s} \# g_{t}\right\}_{s, t \in[0,1]}$ to obtain a path $\left\{f_{u} \# g_{u}\right\}_{u \in[0,1]}$, and the same for \#.

One can also derive from Lemma 4.1.1 analogous properties for the exterior cupproducts of tracks.

\subsection{The Exterior Tracks}

For any two maps $f: \Sigma A \rightarrow \Sigma B$ and $g: \Sigma X \rightarrow \Sigma Y$ the suspended exterior cupproducts $\Sigma(f \# g)$ and $\Sigma(f \# g)$ are naturally homotopic to the composite

$$
S^{1} \wedge S^{1} \wedge A \wedge X \stackrel{(23)}{\cong} S^{1} \wedge A \wedge S^{1} \wedge X \stackrel{f \wedge g}{\longrightarrow} S^{1} \wedge B \wedge S^{1} \wedge Y \stackrel{(23)}{\cong} S^{1} \wedge S^{1} \wedge B \wedge Y .
$$

In order to construct homotopies we only need to choose a track from the transposition map (1 2): $S^{1} \wedge S^{1} \rightarrow S^{1} \wedge S^{1}$ to $v \wedge S^{1}$, where $v: S^{1} \rightarrow S^{1}$ is the co-H-inversion defined by $v(z)=z^{-1}$. Here we use the topological group structure of $S^{1}$. The set of all tracks $\left(\begin{array}{ll}1 & 2\end{array}\right) \Rightarrow v \wedge S^{1}$ and $1_{S^{2}} \Rightarrow 1_{S^{2}}$ is a group under horizontal composition. This group is an extension of $\mathbb{Z} / 2$ by $\mathbb{Z}$ with the non-trivial action of $\mathbb{Z} / 2$, compare $[10,6.12]$. Up to isomorphism there is only one extension of this kind, the trivial extension, given by the infinite dihedral group $\mathbb{Z} / 2 * \mathbb{Z} / 2$, hence this group of tracks is generated by two order 2 tracks $\left(\begin{array}{ll}1 & 2\end{array}\right) \Rightarrow v \wedge S^{1}$. One of these two generating tracks $\mathbb{H}:\left(\begin{array}{ll}1 & 2\end{array}\right) \Rightarrow v \wedge S^{1}$ can be constructed as follows. Since $v \wedge S^{1}$ is a homotopy equivalence it is enough to indicate which track is $\mathbb{H}^{\boxminus}\left(v \wedge S^{1}\right): 1_{S^{2}} \Rightarrow(12)\left(v \wedge S^{1}\right)$. The 2-sphere $S^{2}=S^{1} \wedge S^{1}$ is a quotient of the square $[-1,1]^{2}$ by the map $[-1,1]^{2} \rightarrow$ $S^{1} \wedge S^{1}:(x, y) \mapsto(\exp \pi i(1+x), \exp \pi i(1+y))$. The map $(12)\left(v \wedge S^{1}\right)$ is induced by the $90^{\circ}$ twist (counterclockwise) in the square, so we obtain $\mathbb{H}^{\boxminus}\left(v \wedge S^{1}\right)$ by using the homeomorphism from the square to the radius $\sqrt{2}$ circle projecting from the origin

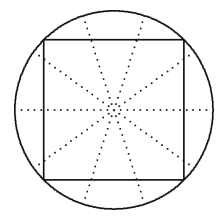

and twisting continuously the circle $90^{\circ}$ counterclockwise.

Definition 4.2.1 The exterior tracks

$$
\begin{aligned}
& \mathbb{H}_{f, g}^{\#}: \Sigma(f \# g) \Rightarrow(23)(f \wedge g)(23), \\
& \mathbb{H}_{f, g}^{\#}: \Sigma(f \# g) \Rightarrow(23)(f \wedge g)(23),
\end{aligned}
$$

are given by the following diagram in Top*, where the 2-cells without a track arrow $\Rightarrow$ are strictly commutative. 


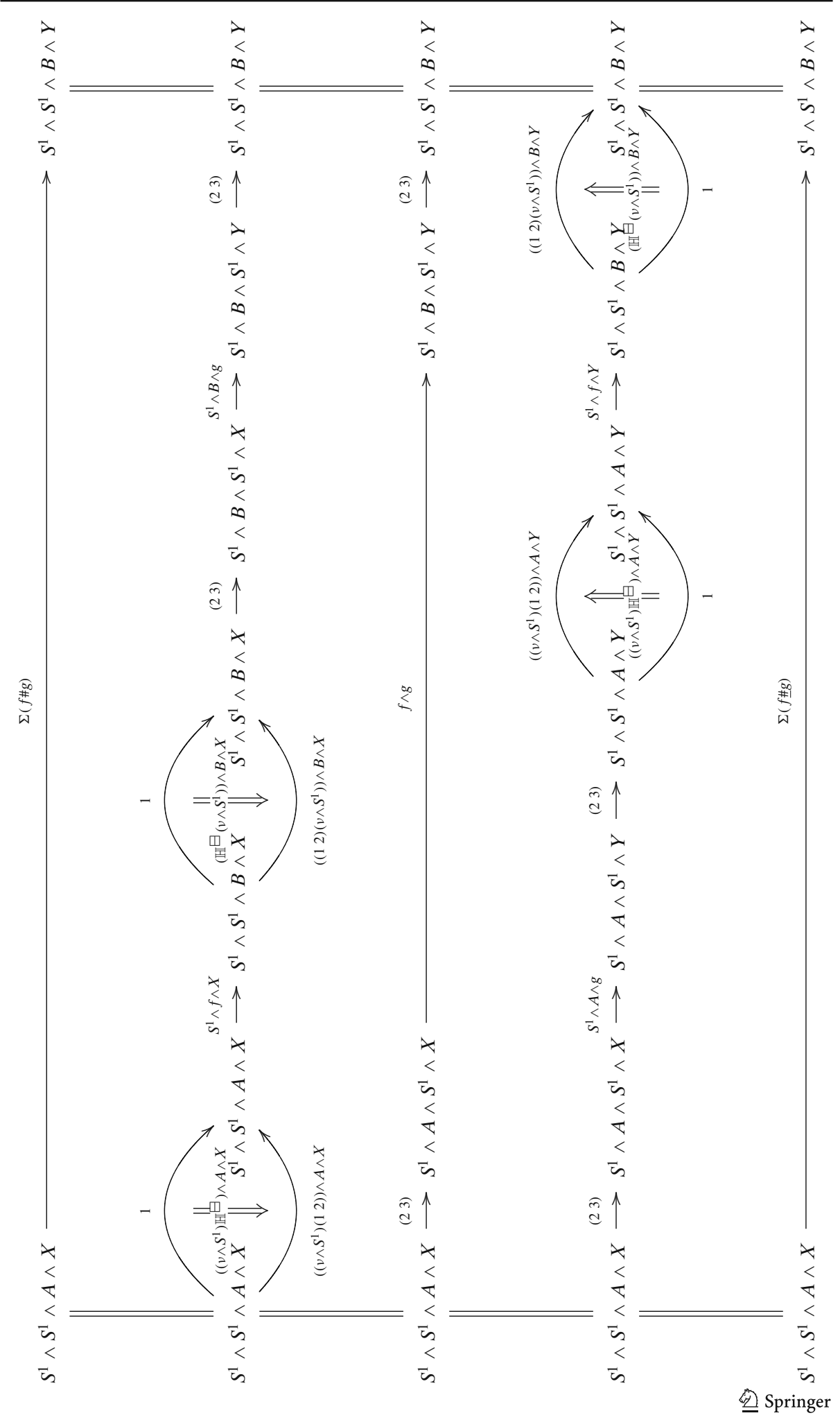


i.e. the tracks $\mathbb{H}_{f, g}^{\#}$ and $\mathbb{H}_{f, g}^{\#}$ are the following composite tracks.

$$
\begin{aligned}
\mathbb{H}_{f, g}^{\#} & =(23)\left(S^{1} \wedge B \wedge g\right)(23)\left(\left(\mathbb{H}^{\boxminus}\left(v \wedge S^{1}\right)\right) \wedge B \wedge X\right)\left(S^{1} \wedge f \wedge X\right)\left(\left(\left(\nu \wedge S^{1}\right) \mathbb{H}^{\boxminus}\right) \wedge A \wedge X\right) \\
& =(23)\left(S^{1} \wedge B \wedge g\right)(23)\left(\mathbb{H}^{\boxminus} \wedge B \wedge X\right)\left(S^{1} \wedge f \wedge X\right)\left(\mathbb{H}^{\boxminus} \wedge A \wedge X\right), \\
\mathbb{H}_{f, g}^{\#} & =\left(\left(\mathbb{H}^{\boxminus}\left(v \wedge S^{1}\right)\right) \wedge B \wedge Y\right)\left(S^{1} \wedge f \wedge Y\right)\left(\left(\left(v \wedge S^{1}\right) \mathbb{H}^{\boxminus}\right) \wedge A \wedge Y\right)(23)\left(S^{1} \wedge A \wedge g\right)(23) \\
& =\left(\mathbb{H}^{\boxminus} \wedge B \wedge Y\right)\left(S^{1} \wedge f \wedge Y\right)\left(\mathbb{H}^{\boxminus} \wedge A \wedge Y\right)(23)\left(S^{1} \wedge A \wedge g\right)(23) .
\end{aligned}
$$

In the next proposition we show elementary properties of the exterior tracks that are relevant for the definition of the smash product operation on secondary homotopy groups. They are analogous to the properties of exterior cup-products in Lemma 4.1.1.

Lemma 4.2.2 The exterior tracks satisfy the following formulas for suspensions

(1) $\mathbb{H}_{\Sigma f^{\prime}, g}^{\#}=0_{\Sigma\left((12)\left(f^{\prime} \wedge g\right)(12)\right)}^{\square}=\mathbb{H} \frac{\#}{f^{\prime}, g}$,

(2) $\mathbb{H}_{f, \Sigma g^{\prime}}^{\#}=\left(\mathbb{H}^{\boxminus} \wedge B \wedge Y\right)\left(S^{1} \wedge f \wedge g^{\prime}\right)\left(\mathbb{H}^{\boxminus} \wedge A \wedge X\right)=\mathbb{H}_{f, \Sigma g^{\prime}}^{\#}$,

coproducts

(3) $\mathbb{H}_{\left(f_{1}, f_{2}\right), g}^{\#}=\left(\mathbb{H}_{f_{1}, g}^{\#}, \mathbb{H}_{f_{2}, g}^{\#}\right)$,

(4) $\quad \mathbb{H}_{\left(f_{1}, f_{2}\right), g}^{\#}=\left(\mathbb{H}_{f_{1}, g}^{\#}, \mathbb{H}_{f_{2}, g}^{\#}\right)$,

(5) $\mathbb{H}_{f,\left(g_{1}, g_{2}\right)}^{\#}=\left(\mathbb{H}_{f, g_{1}}^{\#}, \mathbb{H}_{f, g_{2}}^{\#}\right)$,

(6) $\quad \mathbb{H}_{f,\left(g_{1}, g_{2}\right)}^{\#}=\left(\mathbb{H}_{f, g_{1}}^{\#}, \mathbb{H}_{f, g_{2}}^{\#}\right)$,

and composition of maps

(7) $\mathbb{H}_{f_{1} f_{2}, g_{1}\left(\Sigma g_{2}^{\prime}\right)}^{\#}=\mathbb{H}_{f_{1}, g_{1}}^{\#} \mathbb{H}_{f_{2}, \Sigma g_{2}^{\prime}}^{\#}$,

(8) $\mathbb{H}_{\left(\Sigma f_{1}^{\prime}\right) f_{2}, g_{1} g_{2}}^{\#}=\mathbb{H}_{\Sigma f_{1}^{\prime}, g_{1}}^{\#} \mathbb{H}_{f_{2}, g_{2}}^{\#}$,

(9) $\quad \mathbb{H}_{f_{1}\left(\Sigma f_{2}^{\prime}\right), g_{1} g_{2}}^{\#}=\mathbb{H}_{f_{1}, g_{1}}^{\#} \mathbb{H}_{\Sigma}^{\#} f_{2}^{\prime}, g_{2}$,

(10) $\quad \mathbb{H}_{f_{1} f_{2},\left(\Sigma g_{1}^{\prime}\right) g_{2}}^{\#}=\mathbb{H}_{f_{1}, \Sigma g_{1}^{\prime}}^{\#} \mathbb{H}_{f_{2}, g_{2}}^{\#}$.

They satisfy the following associativity rules.

(11) $\left(\mathbb{H}_{f, g}^{\#} \wedge h\right)\left(\Sigma \mathbb{H}_{f \# g, h}^{\#}\right)=\left(f \wedge \mathbb{H}_{g, h}^{\#}\right)\left(\Sigma \mathbb{H}_{f, g \# h}^{\#}\right): \Sigma^{2}(f \# g \# h) \Rightarrow f \wedge g \wedge h$,

(12) $\left(\mathbb{H}_{f, g}^{\#} \wedge h\right)\left(\Sigma \mathbb{H}_{f \# g, h}^{\#}\right)=\left(f \wedge \mathbb{H}_{g, h}^{\#}\right)\left(\Sigma \mathbb{H}_{f, g \# h}^{\#}\right): \Sigma^{2}(f \# g \# h) \Rightarrow f \wedge g \wedge h$.

These properties follow easily from the definition of exterior tracks above and from the fact that $\mathbb{H} \mathbb{H}=0_{1_{s^{2}}}^{\square}$ is the trivial track. In the right hand side of the equalities (11) and (12) there are some permutations involved that we have omitted. 
For suspended maps $\Sigma^{n-1} f: \Sigma^{n} A \rightarrow \Sigma^{n} B, \Sigma^{m-1} g: \Sigma^{m} X \rightarrow \Sigma^{m} Y$ we define the tracks $\mathbb{H}_{n, f, m, g}^{\#}, \mathbb{H}_{n, f, m, g}^{\#}$ from $\Sigma^{n+m-1}(f \# g), \Sigma^{n+m-1}(f \# g)$, respectively, to

$$
S^{n} \wedge S^{m} \wedge A \wedge X \cong S^{n} \wedge A \wedge S^{m} \wedge X \stackrel{\Sigma^{n-1} f \wedge \Sigma^{m-1} g}{\longrightarrow} S^{n} \wedge B \wedge S^{m} \wedge Y \cong S^{n} \wedge S^{m} \wedge B \wedge Y
$$

as

$$
\begin{aligned}
& S^{n-1} \wedge S^{1} \wedge S^{m-1} \wedge S^{1} \wedge A \wedge X \\
& \cong \text { (23) } \\
& S^{n-1} \wedge S^{m-1} \wedge S^{1} \wedge S^{1} \wedge A \wedge X \\
& \Sigma^{n+m-1}(f \# g)\left(\stackrel{\Sigma^{n+m-2} \mathbb{H}_{f, g}^{\#}}{\rightleftharpoons}\right) \Sigma^{n+m-2}((23)(f \wedge g)(23)) \\
& S^{n-1} \wedge S^{m-1} \wedge S^{1} \wedge S^{1} \wedge B \wedge Y
\end{aligned}
$$

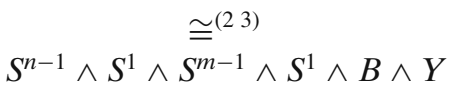

and similarly for \#. Notice that the last spherical coordinate in these smash products is always the same one. This is relevant in connection with Lemma 4.4.2 below. The tracks $\mathbb{H}_{n, f, m, g}^{\#}, \mathbb{H}_{n, f, m, g}^{\#}$ satisfy properties analogous to Lemma 4.2.2 that we do not restate. They also satisfy the following further properties.

Lemma 4.2.3 Given tracks $F: \Sigma^{n-1} f_{1} \Rightarrow \Sigma^{n-1} f_{2}$ and $G: \Sigma^{m-1} g_{1} \Rightarrow \Sigma^{m-1} g_{2}$ between maps $\Sigma^{n-1} f_{i}: \Sigma^{n} A \rightarrow \Sigma^{n} B$ and $\Sigma^{m-1} g_{i}: \Sigma^{m} A \rightarrow \Sigma^{m} B, i=1,2$, the following equalities are satisfied

(1) $(F \wedge G) \square \mathbb{H}_{n, f_{1}, m, g_{1}}^{\#}=\mathbb{H}_{n, f_{2}, m, g_{2}}^{\#} \square(\Sigma(F \# G)): \Sigma^{n+m-1}\left(f_{1} \# g_{1}\right) \Rightarrow \Sigma^{n+m-2}\left(f_{2} \wedge g_{2}\right)$, (2) $(F \wedge G) \square \mathbb{H}_{n, f_{1}, m, g_{1}}^{\#}=\mathbb{H}_{n, f_{2}, m, g_{2}}^{\#} \square(\Sigma(F \# G)): \Sigma^{n+m-1}\left(f_{1} \# g_{1}\right) \Rightarrow \Sigma^{n+m-2}\left(f_{2} \wedge g_{2}\right)$.

Here the track $F \wedge G$ in (1) and (2) needs to be altered by permutations according to Eq. 32. Moreover $\Sigma(F \# G)$ and $\Sigma(F \# G)$ need also to be altered by permutations as follows. The track $\Sigma(F \# G)$ should actually be

$$
\begin{aligned}
& S^{n-1} \wedge S^{1} \wedge S^{m-1} \wedge S^{1} \wedge A \wedge X \\
& \cong(12) \\
& S^{1} \wedge S^{n-1} \wedge S^{m-1} \wedge S^{1} \wedge A \wedge X \\
& \Sigma^{n+m-1}\left(f_{1} \# g_{1}\right)(\stackrel{\Sigma(F \# G)}{\Longrightarrow}) \Sigma^{n+m-1}\left(f_{2} \# g_{2}\right) \\
& S^{1} \wedge S^{n-1} \wedge S^{m-1} \wedge S^{1} \wedge B \wedge Y \\
& \cong^{(12)} \\
& S^{n-1} \wedge S^{1} \wedge S^{m-1} \wedge S^{1} \wedge B \wedge Y
\end{aligned}
$$

and similarly $\Sigma(F \# G)$. Notice that the last spherical coordinate remains always in the same place in this diagram. This is again relevant in connection with Lemma 4.4.2 below. 


\subsection{The Construction of the Smash Product Operation}

In this section we define the smash product morphism in $\mathbf{q p m}$ which appears in the statement of Theorem 3.2.1. In the last two sections we establish the properties which show that the definition given here is indeed consistent with the definition of the tensor product of quadratic pair modules.

The secondary homotopy groups $\pi_{n, *} X, n \geq 0$, of a pointed space $X$ were introduced in [9].

For $n=0, \pi_{0, *} X$ is the fundamental pointed groupoid of $X$. We denote by $\pi_{0,0} X$ the pointed set of objects, which can be regarded as the set of pointed maps $S^{0} \rightarrow X$, and by $\pi_{0,1} X$ to the set of morphisms. Such a morphism $\alpha: x \rightarrow y$ is a track $\alpha: x \Rightarrow y$ between pointed maps $x, y: S^{0} \rightarrow Y$.

For $n=1, \pi_{1, *} X$ is a crossed module

$$
\text { ว: } \pi_{1,1} X \longrightarrow \pi_{1,0} X=\langle\Omega X\rangle .
$$

In particular $\pi_{1,0} X$ acts on the right on $\pi_{1,1} X$.

For $n \geq 2, \pi_{n, *} X$ is a reduced quadratic module in the sense of [2]

$$
\otimes^{2}\left(\pi_{n, 0} X\right)_{a b} \stackrel{\omega}{\longrightarrow} \pi_{n, 1} X \stackrel{\partial}{\longrightarrow} \pi_{n, 0} X=\left\langle\Omega^{n} X\right\rangle_{n i l},
$$

which is stable for $n \geq 3$.

For all $n \geq 1$ the elements of $\pi_{n, 1} X$ are equivalence classes $[f, F]$ represented by a map

$$
f: S^{1} \longrightarrow \vee_{\Omega^{n} X} S^{1}
$$

and a track

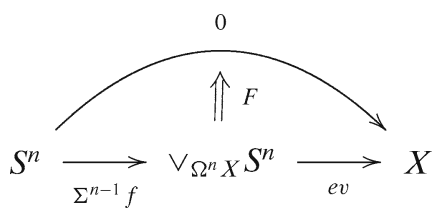

where $e v$ is the obvious evaluation map. Recall from Section 3.1 the notation $S_{X}^{n}=$ $\vee_{\Omega^{n} X} S^{n}$. Two elements $[f, F],[g, G] \in \pi_{n, 1} X$ coincide provided there is a diagram like Eq. 19 parting to the trivial track with $\operatorname{Hopf}(N)=0$ for $n \geq 2$ and no conditions on $N$ for $n=1$. We refer the reader to [9] for further details on the construction of the algebraic structure of $\pi_{n, *} X$.

According to the definition of additive secondary homotopy groups of a pointed space given in Remark 3.1.3 the quadratic pair module $\Pi_{n, *} X$ looks as follows.

$$
\Pi_{n, *} X=\left(\Pi_{n, 1} X \Pi_{n, 0} X=\left\langle\Omega^{n} X\right\rangle_{n i l}\right)
$$

Here $H$ is always defined as in Eq. 10 . 
For $n \geq 3, \Pi_{n, 1} X=\pi_{n, 1} X$ and $P=\omega \tau_{\otimes}$ and $\partial$ in Eq. 34 are given by the homomorphisms in Eq. 33.

For $n=2$ the group $\Pi_{2,1} X$ is the quotient of $\pi_{2,1} X$ by the relations

$$
P(a \otimes b+b \otimes a)=0 ; \quad a, b \in\left(\pi_{2,0} X\right)_{a b} ;
$$

and $P$ and $\partial$ in Eq. 34 are induced by $\omega \tau_{\otimes}$ and $\partial$ in Eq. 33 respectively.

For $n=1, \Pi_{1,1} X$ is the quotient of the group

$$
\pi_{1,1} X \times\left(\hat{\otimes}^{2}\left(\pi_{1,0} X\right)_{a b}\right)
$$

by the relations

$$
\left(-[f, F]+[f, F]^{x}, 0\right)=(0, x \hat{\otimes} \partial[f, F]) ; \quad[f, F] \in \pi_{1,1} X, x \in \pi_{1,0} X ;
$$

$P(a \otimes b)=(0, a \hat{\otimes} b)$ for $a, b \in\left(\pi_{1,0} X\right)_{a b} ;$ and $\partial([f, F], x \hat{\otimes} y)=\partial[f, F]-y-x+$ $y+x$ in $\langle\Omega X\rangle_{\text {nil }}$ for $[f, F] \in \pi_{1,1} X$ and $x, y \in \pi_{1,0} X$.

Finally for $n=0$ the group $\Pi_{0,1} X$ is the quotient of

$$
\left\langle\pi_{0,1} X\right\rangle_{\text {nil }} \times\left(\hat{\otimes}^{2} \mathbb{Z}\left[\pi_{0,0} X\right]\right)
$$

by the relations

$$
\left(\left[\alpha, \alpha^{\prime}\right], 0\right)=\left(0,\left(-x^{\prime}+y^{\prime}\right) \hat{\otimes}(-x+y)\right)
$$

for all morphisms $\alpha: x \rightarrow y$ and $\alpha^{\prime}: x^{\prime} \rightarrow y^{\prime}$ in $\pi_{0, *} X$,

$$
(\alpha \beta, 0)=(\beta+\alpha, 0),
$$

for all composable morphisms $\bullet \stackrel{\beta}{\rightarrow} \bullet \stackrel{\alpha}{\rightarrow} \bullet$ in $\pi_{0, *} X, P(a \otimes b)=(0, a \hat{\otimes} b)$ for $a, b \in$ $\pi_{0,0} X$; and $\partial(\alpha, a \hat{\otimes} b)=-x+y+[b, a]$ for $a, b \in \pi_{0,0} X$ and $\alpha: x \rightarrow y$ in $\pi_{0,1} X$.

We denote by $\Pi_{n,(0)} X$ and $\Pi_{n,(1)} X$ the square groups

$$
\begin{aligned}
& \Pi_{n,(0)} X=\left(\Pi_{n, 0} X \underset{\partial P}{\stackrel{H}{\rightleftarrows}} \Pi_{n, e e} X\right), \\
& \Pi_{n,(1)} X=\left(\Pi_{n, 1} X \underset{P}{\stackrel{H \partial}{\rightleftarrows}} \Pi_{n, e e} X\right),
\end{aligned}
$$

defining the quadratic pair module $\Pi_{n, *} X$.

Definition 4.3.1 The smash product operation for the additive secondary homotopy groups of two pointed spaces $X, Y$ is given by morphisms in $\mathbf{q p m}, n, m \geq 0$,

$$
\Pi_{n, *} X \odot \Pi_{m, *} Y \stackrel{\wedge}{\longrightarrow} \Pi_{n+m, *}(X \wedge Y) .
$$

These morphisms are induced by square group morphisms, $n, m \geq 0,0 \leq i, j, i+j \leq 1$,

$$
\Pi_{n,(i)} X \odot \Pi_{m,(j)} Y \stackrel{\wedge}{\longrightarrow} \Pi_{n+m,(i+j)}(X \wedge Y) .
$$

defined as follows:

For $i=j=0$ morphism Eq. 36 is the composition

$$
\mathbb{Z}_{\text {nil }}\left[\Omega^{n} X\right] \odot \mathbb{Z}_{\text {nil }}\left[\Omega^{m} Y\right] \cong \mathbb{Z}_{\text {nil }}\left[\left(\Omega^{n} X\right) \wedge\left(\Omega^{m} Y\right)\right] \stackrel{\mathbb{Z}_{\text {nil }}[\wedge]}{\longrightarrow} \mathbb{Z}_{\text {nil }}\left[\Omega^{n+m}(X \wedge Y)\right]
$$


of the isomorphism in Proposition 2.3.2 and the morphism induced by the map between discrete pointed sets

$$
\wedge:\left(\Omega^{n} X\right) \wedge\left(\Omega^{m} Y\right) \longrightarrow \Omega^{m+n}(X \wedge Y), \quad n, m \geq 0,
$$

defined by

$$
\left(f: S^{n} \rightarrow X\right) \wedge\left(g: S^{m} \rightarrow Y\right) \mapsto\left(f \wedge g: S^{n+m} \rightarrow X \wedge Y\right) .
$$

All morphisms in Eq. 36 coincide in the ee-term.

Suppose now that $n, m \geq 1$.

For $i=0$ and $j=1$, an element $g \odot[f, F]$ with $g \in \Pi_{n, 0} X$ and $[f, F] \in \Pi_{m, 1} Y$ is sent by Eq. 36 to the element $g \wedge[f, F] \in \Pi_{n+m, 1}(X \wedge Y)$ represented by the map

$$
S^{1} \stackrel{\bar{g} \# f}{\longrightarrow} \vee_{\left(\Omega^{n} X\right) \wedge\left(\Omega^{m} Y\right)} S^{1} \stackrel{\Sigma \wedge}{\longrightarrow} \vee_{\Omega^{n+m}(X \wedge Y)} S^{1},
$$

where $\bar{g}: S^{1} \rightarrow \vee_{\Omega^{n} X} S^{1}$ is any map with $\left(\pi_{1} \bar{g}\right)_{\text {nil }}(1)=g$ and the second arrow is the suspension of $\wedge$ in Eq. 37, and by the track

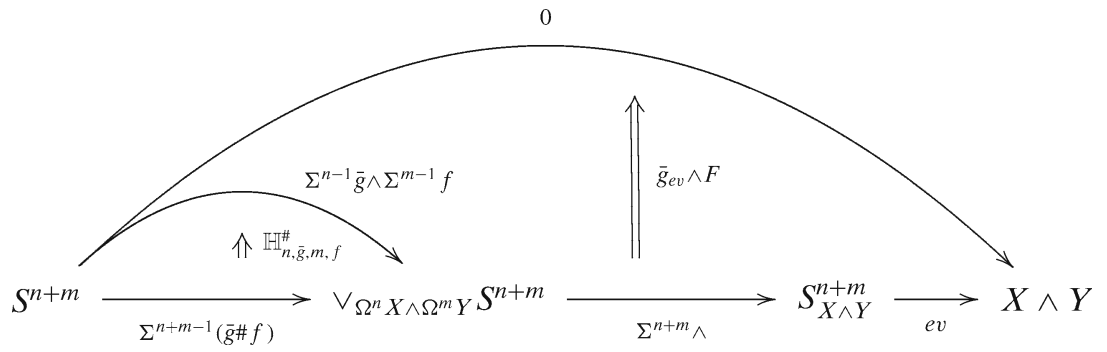

i.e.

$$
g \wedge[f, F]=\left[(\Sigma \wedge)(\bar{g} \# f),\left(\bar{g}_{e v} \wedge f\right) \square\left(\operatorname{ev}\left(\Sigma^{n+m} \wedge\right) \mathbb{H}_{n, \bar{g}, m, f}^{\#}\right)\right]
$$

Here, and in the following three cases, the smash products of maps and tracks need to be altered by permutations according to Eq. 32 .

In a similar way the element $g \subseteq[f, F]$ is sent by Eq. 36 to $g \wedge[f, F] \in \Pi_{n+m, 1}(X \wedge Y)$, given by the map

$$
S^{1} \stackrel{\bar{g} \# f}{\longrightarrow} \vee_{\left(\Omega^{n} X\right) \wedge\left(\Omega^{m} Y\right)} S^{1} \stackrel{\Sigma \wedge}{\longrightarrow} \vee_{\Omega^{n+m}(X \wedge Y)} S^{1}
$$

and by the track

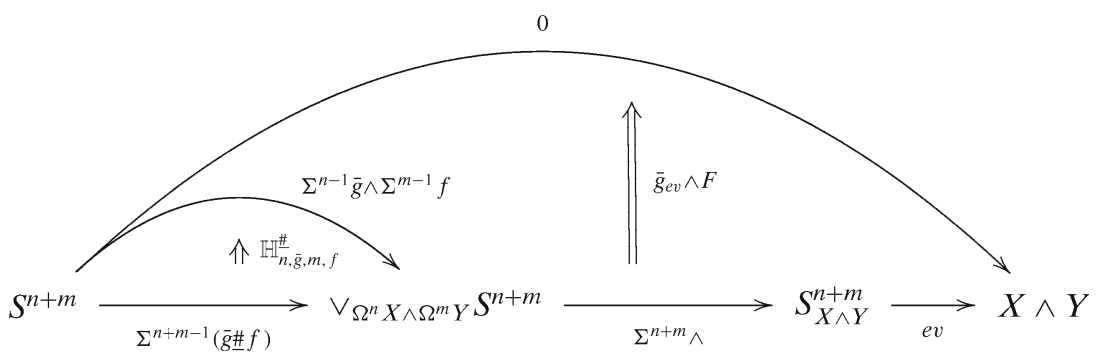

i.e.

$$
g \wedge[f, F]=\left[(\Sigma \wedge)(\bar{g} \# f),\left(\bar{g}_{e v} \wedge f\right) \square\left(e v\left(\Sigma^{n+m} \wedge\right) \mathbb{H}_{n, \bar{g}, m, f}^{\#}\right)\right]
$$


For $i=1$ and $j=0$ the generator $[f, F] \odot g$ with $[f, F] \in \Pi_{n, 1} X$ and $g \in \Pi_{m, 0} Y$ is sent by Eq. 36 to the element $[f, F] \wedge g \in \Pi_{n+m, 1}(X \wedge Y)$ represented by the map

$$
S^{1} \stackrel{f \# \bar{g}}{\longrightarrow} \vee_{\left(\Omega^{n} X\right) \wedge\left(\Omega^{m} Y\right)} S^{1} \stackrel{\Sigma \wedge}{\longrightarrow} \vee_{\Omega^{n+m}(X \wedge Y)} S^{1},
$$

where $\bar{g}: S^{1} \rightarrow \vee_{\Omega^{m} Y} S^{1}$ is any map with $\left(\pi_{1} \bar{g}\right)_{n i l}(1)=g$, and the track

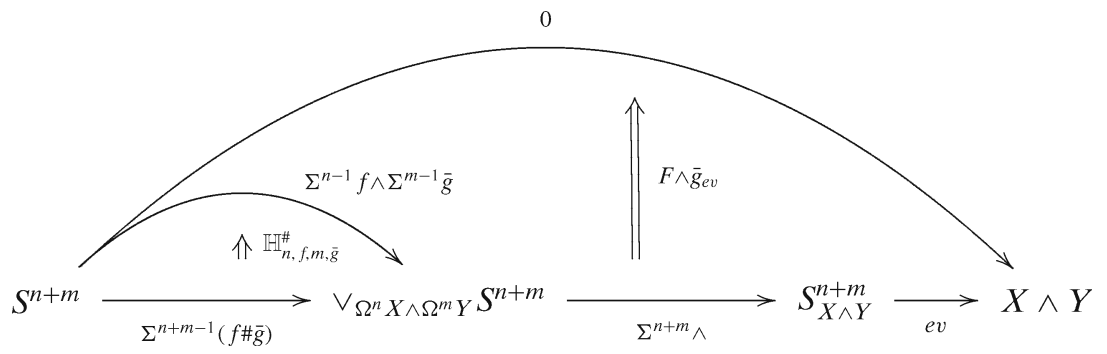

i.e.

$$
[f, F] \wedge g=\left[(\Sigma \wedge)(f \# \bar{g}),\left(F \wedge \bar{g}_{e v}\right) \square\left(e v\left(\Sigma^{n+m} \wedge\right) \mathbb{H}_{n, f, m, \bar{g}}^{\#}\right)\right] .
$$

The element $[f, F] \ominus g$ is sent by Eq. 36 to $[f, F] \wedge g \in \Pi_{n+m, 1}(X \wedge Y)$ given by the map

$$
S^{1} \stackrel{f \# \bar{g}}{\longrightarrow} \vee_{\left(\Omega^{n} X\right) \wedge\left(\Omega^{m} Y\right)} S^{1} \stackrel{\Sigma \wedge}{\longrightarrow} \vee_{\Omega^{n+m}(X \wedge Y)} S^{1}
$$

and the track

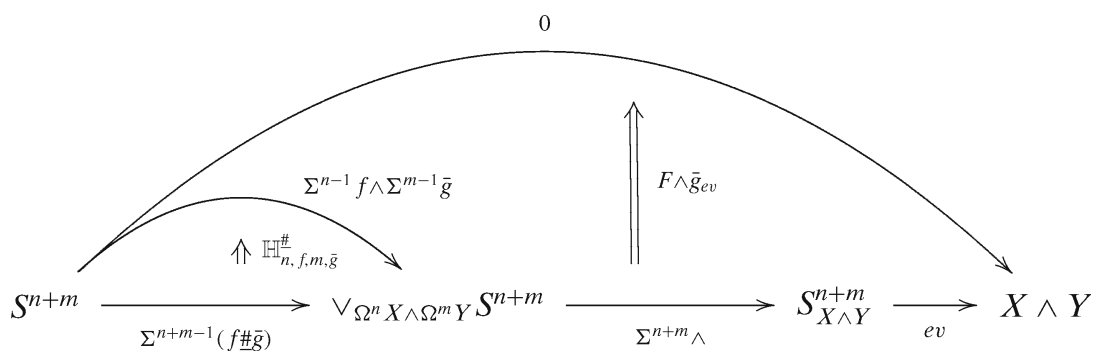

i.e.

$$
[f, F] \wedge g=\left[(\Sigma \wedge)(f \underline{\# \bar{g}}),\left(F \wedge \bar{g}_{e v}\right) \square\left(\operatorname{ev}\left(\Sigma^{n+m} \wedge\right) \mathbb{H}_{n, f, m, \bar{g}}^{\#}\right)\right] .
$$

Suppose now that $n=0$ and $m \geq 0$.

For $i=0$ and $j=1$, an element $g \odot f$ with $g: S^{0} \rightarrow X$ and $f \in \Pi_{m, 1} Y$ is sent by Eq. 36 to $g \wedge f=\left(\Pi_{m, 1}(g \wedge Y)\right)(f) \in \Pi_{m, 1}(X \wedge Y)$.

For $i=1$ and $j=0$, an element $F \odot g$ with $F: f \Rightarrow f^{\prime}$ a track between maps $f, f^{\prime}: S^{0} \rightarrow X$ and $g \in \Pi_{m, 0} Y$ is sent by Eq. 36 to $F \wedge g=\left(\Pi_{m, *}(F \wedge\right.$ $Y))(g) \Pi_{m, 1}(X \wedge Y)$.

Suppose now that $n \geq 0$ and $m=0$.

For $i=0$ and $j=1$, an element $g \odot F$ with $g \in \Pi_{n, 0} X$ and $F: f \Rightarrow f^{\prime}$ a track between maps $f, f^{\prime}: S^{0} \rightarrow Y$ is sent by Eq. 36 to $g \wedge F=\left(\Pi_{n, *}(X \wedge f)\right)(g) \Pi_{n, 1}(X \wedge Y)$.

For $i=1$ and $j=0$, an element $f \odot g$ with $f \in \Pi_{n, 1} X$ and $g: S^{0} \rightarrow Y$ is sent by Eq. 36 to $f \wedge g=\left(\Pi_{m, 1}(X \wedge g)\right)(f) \Pi_{n, 1}(X \wedge Y)$. 
4.4 The Hopf Invariant for Tracks and Smash Products

In this section we prove two lemmas on the Hopf invariant for tracks defined in $[9,3.3]$ which will be useful to check the properties of the smash product operation on secondary homotopy groups.

The first lemma computes the Hopf invariant of a track smashed with a discrete set.

Lemma 4.4.1 Let $f, g: S^{1} \wedge A \rightarrow S^{1} \wedge B$ be maps between suspensions of discrete pointed sets $A, B$; let $F: S^{1} \wedge f \Rightarrow S^{1} \wedge g$ be a track; and let $X$ be another discrete pointed set. Then the following equations hold

(1) $\operatorname{Hopf}(F \wedge X)=\left(1 \otimes \tau_{\otimes} \otimes 1\right)(H o p f(F) \otimes \Delta)$,

(2) $\operatorname{Hopf}(X \wedge f)=\left(1 \otimes \tau_{\otimes} \otimes 1\right)(\Delta \otimes H o p f(F))$.

In particular the smash product of a track with trivial Hopf invariant and a discrete pointed set has always a trivial Hopf invariant.

This lemma follows easily from the elementary properties of the Hopf invariant for tracks in $[9,3]$.

The second lemma computes the effect of conjugation by an automorphism of a sphere on certain tracks.

Lemma 4.4.2 Let $f, g: S^{1} \wedge A \rightarrow S^{1} \wedge B$ be maps between suspensions of discrete pointed sets $A, B$; let $F: S^{n-1} \wedge f \Rightarrow S^{n-1} \wedge g$ be a track; and let $\alpha: S^{n-1} \cong S^{n-1}$ be a homeomorphism. Then $\left(\alpha \wedge S^{1} \wedge B\right) F\left(\alpha^{-1} \wedge S^{1} \wedge A\right)$ is a track with trivial Hopf invariant if and only if $F$ has trivial Hopf invariant. Moreover, if $\alpha$ has degree 1 or $n \geq 3$ then

$$
F=\left(\alpha \wedge S^{1} \wedge B\right) F\left(\alpha^{-1} \wedge S^{1} \wedge A\right)
$$

Proof Let us denote also by $F: I_{+} \wedge S^{n-1} \wedge S^{1} \wedge A \rightarrow S^{n-1} \wedge S^{1} \wedge B$ to a map representing the track $F$. The adjoint map of pairs

$$
\begin{aligned}
& \operatorname{ad}\left(\left(\alpha \wedge S^{1} \wedge B\right) F\left(I_{+} \wedge \alpha^{-1} \wedge S^{1} \wedge A\right)\right): \\
& \quad\left(I_{+} \wedge S^{1} \wedge A,\left(S^{1} \wedge A\right) \vee\left(S^{1} \wedge A\right)\right) \longrightarrow\left(\Omega^{n-1}\left(S^{n-1} \wedge S^{1} \wedge B\right), S^{1} \wedge B\right)
\end{aligned}
$$

used to define the Hopf invariant coincides with the composite

$$
\begin{gathered}
\left(I_{+} \wedge S^{1} \wedge A,\left(S^{1} \wedge A\right) \vee\left(S^{1} \wedge A\right)\right) \\
\downarrow \operatorname{ad}(F) \\
\left(\Omega^{n-1}\left(S^{n-1} \wedge S^{1} \wedge B\right), S^{1} \wedge B\right) \\
\quad \downarrow \operatorname{map}_{*}\left(\alpha^{-1}, \alpha \wedge S^{1} \wedge B\right) \\
\left(\Omega^{n-1}\left(S^{n-1} \wedge S^{1} \wedge B\right), S^{1} \wedge B\right)
\end{gathered}
$$

The map $\operatorname{map}_{*}\left(\alpha^{-1}, \alpha \wedge S^{1} \wedge B\right)$ is a homeomorphism, hence the first part of the lemma follows from the very definition of the Hopf invariant for tracks. 
The homeomorphism $\operatorname{map}_{*}\left(\alpha^{-1}, \alpha \wedge S^{1} \wedge B\right)$ restricts to the identity on $S^{1} \wedge B$. Moreover, if $\alpha$ has degree 1 or $n \geq 3$ then this homeomorphism is compatible with the $H$-multiplication of the $(n-1)$-fold loop space up to homotopy, and therefore with the Pontrjagin product. In particular by the definition and elementary properties of the Hopf invariant for tracks

$$
\operatorname{Hopf}(F)=\operatorname{Hopf}\left(\left(\alpha \wedge S^{1} \wedge B\right) F\left(\alpha^{-1} \wedge S^{1} \wedge A\right)\right)
$$

and hence

$$
F=\left(\alpha \wedge S^{1} \wedge B\right) F\left(\alpha^{-1} \wedge S^{1} \wedge A\right)
$$

\subsection{Hopf Invariant Computations Related to Exterior Tracks}

In this section we perform two Hopf invariant computations for tracks. The first computation is connected with axiom (7) in the definition of the tensor product of square groups, see Definition 2.2.1. The second one is connected with the commutativity rule for the smash product operation on additive secondary homotopy groups, see Theorem 3.2.3. Both computations are crucial steps towards the proof of the main results of this paper stated in Section 3.2. They show that the algebraic structures described in Sections 2 and 3 are the right algebraic structures to describe the smash product operation.

First of all we define a concept which will be useful for computations.

Definition 4.5.1 Let nil be the category of free groups of nilpotency class 2 and let $\Phi: \mathbf{A b} \rightarrow \mathbf{A b}$ be a functor. The G-group of $\Phi$ is the class $\mathrm{G}(\Phi)$ of all functions $\chi$ sending two morphisms $f:\langle A\rangle_{\text {nil }} \rightarrow\langle B\rangle_{n i l}, g:\langle X\rangle_{\text {nil }} \rightarrow\langle Y\rangle_{\text {nil }}$ in nil to a homomorphism

$$
\chi(f, g): \mathbb{Z}[A] \otimes \mathbb{Z}[X] \longrightarrow \Phi(\mathbb{Z}[B] \otimes \mathbb{Z}[Y])
$$

in such a way that if $f, f_{i}, g, g_{i}$ are morphisms in nil and $f^{\prime}, f_{i}^{\prime}, g^{\prime}, g_{i}^{\prime}$ are maps between pointed sets $i=1,2$ then

(1) $\chi\left(\left\langle f^{\prime}\right\rangle_{\text {nil }}, g\right)=0$,

(2) $\chi\left(f,\left\langle g^{\prime}\right\rangle_{\text {nil }}\right)=0$,

(3) $\chi\left(\left(f_{1}, f_{2}\right), g\right)=\left(\chi\left(f_{1}, g\right), \chi\left(f_{2}, g\right)\right)$,

(4) $\chi\left(f,\left(g_{1}, g_{2}\right)\right)=\left(\chi\left(f, g_{1}\right), \chi\left(f, g_{2}\right)\right)$,

(5) $\chi\left(f_{1}\left\langle f_{2}^{\prime}\right\rangle_{\text {nil }}, g_{1}\left\langle g_{2}^{\prime}\right\rangle_{\text {nil }}\right)=\chi\left(f_{1}, g_{1}\right)\left(\mathbb{Z}\left[f_{2}^{\prime}\right] \otimes \mathbb{Z}\left[g_{2}^{\prime}\right]\right)$,

(6) $\chi\left(\left\langle f_{1}^{\prime}\right\rangle_{\text {nil }} f_{2},\left\langle g_{1}^{\prime}\right\rangle_{\text {nil }} g_{2}\right)=\Phi\left(\mathbb{Z}\left[f_{1}^{\prime}\right] \otimes \mathbb{Z}\left[g_{1}^{\prime}\right]\right) \chi\left(f_{2}, g_{2}\right)$.

A natural transformation $\zeta: \Phi \Rightarrow \Psi$ between functors $\Phi, \Psi: \mathbf{A b} \rightarrow \mathbf{A b}$ induces a function $\mathrm{G}(\zeta): \mathrm{G}(\Phi) \rightarrow \mathrm{G}(\Psi)$ in the obvious way.

If $\mathrm{G}(\Phi)$ is a set then it is an abelian group by addition of abelian group homomorphisms. If $\mathrm{G}(\Psi)$ is also a set then $\mathrm{G}(\zeta)$ is an abelian group homomorphism.

Many functors have a G-group which is a set, see for example Lemma 4.5.6 below. Alternatively one can define G-groups by using a small subcategory of nil to obtain always sets. We therefore do not care about set theoretic subtleties in what follows. 
The following lemma shows examples of non-trivial elements in the G-group of the reduced tensor square.

Lemma 4.5.2 There are elements, $n, m \geq 0$,

$$
\bar{\sigma} \tau_{\otimes}\left(1 \otimes \tau_{\otimes} \otimes 1\right)(H \otimes T H), \quad-\bar{\sigma}\left(\begin{array}{c}
(-1)^{n m} \\
2
\end{array}\right) H(\#) \in \mathrm{G}\left(\hat{\otimes}^{2}\right)
$$

which evaluated at $f:\langle A\rangle_{\text {nil }} \rightarrow\langle B\rangle_{\text {nil }}, g:\langle X\rangle_{\text {nil }} \rightarrow\langle Y\rangle_{\text {nil }}$ send an element $a \otimes x \in$ $\mathbb{Z}[A] \otimes \mathbb{Z}[X]$ with $a \in A$ and $x \in X$ to

$$
\begin{gathered}
\bar{\sigma} \tau_{\otimes}\left(1 \otimes \tau_{\otimes} \otimes 1\right)(H(f(a)) \otimes T H(g(x))), \\
-\bar{\sigma}\left(\begin{array}{c}
(-1)^{n m} \\
2
\end{array}\right) H(f(a) \# g(x)),
\end{gathered}
$$

in $\hat{\otimes}^{2}(\mathbb{Z}[B] \otimes \mathbb{Z}[Y])$ respectively.

Properties (1)-(6) in Definition 4.5.1 are easy to check in these cases.

The following lemma is left as an exercise for the reader.

Lemma 4.5.3 Given three functors $\Phi, \Psi, \Gamma: \mathbf{A b} \rightarrow \mathbf{A b}$ and a natural exact sequence

$$
\Phi \stackrel{\zeta}{\hookrightarrow} \Psi \stackrel{\xi}{\longrightarrow} \Gamma
$$

the sequence

$$
\mathrm{G}(\Phi) \stackrel{\mathrm{G}(\zeta)}{\hookrightarrow} \mathrm{G}(\xi) \stackrel{\mathrm{G}(\xi)}{\longrightarrow} \mathrm{G}(\Gamma)
$$

is exact.

This lemma can be applied to the natural exact sequence

$$
A \otimes \mathbb{Z} / 2 \stackrel{\bar{\tau}}{\hookrightarrow} \hat{\otimes}^{2} A \stackrel{q}{\rightarrow} \wedge^{2} A,
$$

where $\wedge^{2} A$ is the exterior square of $A, \bar{\tau}(a)=\bar{\sigma}(a \otimes a)$ and $q \bar{\sigma}(a \otimes b)=a \wedge b$.

Now we define elements in the G-group of the reduced tensor square by using the exterior tracks and the Hopf invariant for tracks.

In the rest of this section $A, B, X, Y$ will always be pointed discrete sets. Given maps $f: \Sigma A \rightarrow \Sigma B, g: \Sigma X \rightarrow \Sigma Y$, we define the following abelian group homomorphism as a Hopf invariant for tracks

$$
K(f, g)=\bar{\sigma} \operatorname{Hopf}\left(\left(\mathbb{H}_{f, g}^{\#}\right)^{\boxminus} \square \mathbb{H}_{f, g}^{\#}\right): \mathbb{Z}[A] \otimes \mathbb{Z}[X] \longrightarrow \hat{\otimes}^{2}(\mathbb{Z}[B] \otimes \mathbb{Z}[Y]) .
$$

Proposition 4.5.4 The homomorphism $K(f, g)$ defined above only depends on $\left(\pi_{1} f\right)_{\text {nil }}$ and $\left(\pi_{1} g\right)_{\text {nil }}$. Moreover, $K \in \mathrm{G}\left(\hat{\otimes}^{2}\right)$. Furthermore,

$$
\mathrm{G}(q)(K)=\mathrm{G}(q)\left(\bar{\sigma} \tau_{\otimes}\left(1 \otimes \tau_{\otimes} \otimes 1\right)(H \otimes T H)\right) .
$$

Proof By $[9,3.6(5)]$ and Lemma 4.4.2

悬 Springer

$$
K(f, g)=\operatorname{Hopf}\left(\left(\mathbb{H}_{n, f, m, g}^{\#}\right)^{\boxminus} \square \mathbb{H}_{n, f, m, g}^{\#}\right)
$$


for $n, m \geq 1$ with $n+m>2$. If $\left(\pi_{1} f\right)_{n i l}=\left(\pi_{1} \bar{f}\right)_{n i l}$ and $\left(\pi_{1} g\right)_{n i l}=\left(\pi_{1} \bar{g}\right)_{n i l}$ then there are tracks $F: \Sigma^{n-1} f \Rightarrow \Sigma^{n-1} \bar{f}, G: \Sigma^{m-1} g \Rightarrow \Sigma^{m-1} \bar{g}$ with trivial Hopf invariant. Moreover, the tracks

$$
\begin{aligned}
& \Sigma(F \# G): \Sigma^{n+m-1}(f \# g) \Rightarrow \Sigma^{n+m-1}(\bar{f} \# \bar{g}), \\
& \Sigma(F \# G): \Sigma^{n+m-1}(f \underline{\# g}) \Rightarrow \Sigma^{n+m-1}(\bar{f} \ddot{\bar{g}}),
\end{aligned}
$$

have trivial Hopf invariant by Lemmas 4.4.1 and 4.4.2, and [9, 3.6].

By Lemma 4.2.3 we have that

$$
\begin{aligned}
\Sigma(F \# G)^{\boxminus} \square\left(\mathbb{H}_{n, \bar{f}, m, \bar{g}}^{\#}\right)^{\boxminus} \square \mathbb{H}_{n, \bar{f}, m, \bar{g}}^{\#} \square \Sigma(F \# G)= & \left(\mathbb{H}_{n, f, m, g}^{\#}\right)^{\boxminus} \square(F \wedge G)^{\boxminus} \\
& \square(F \wedge G) \square \mathbb{H}_{n, f, m, g}^{\#} \\
= & \left(\mathbb{H}_{n, f, m, g}^{\#}\right)^{\boxminus} \square \mathbb{H}_{n, f, m, g}^{\#} .
\end{aligned}
$$

Hence the first part of the statement follows from the elementary properties of the Hopf invariant for tracks in $[9,3]$.

The equation for the images by $q$ follows from $[9,3.6]$, Eq. a, and the equality

$$
\begin{aligned}
& \left(\pi_{1}(f \# g)\right)_{n i l}(a \wedge x)=\left(\pi_{1}(f \# g)\right)_{n i l}(a \wedge x) \\
& +\partial \tau_{\otimes}\left(1 \otimes \tau_{\otimes} \otimes 1\right)\left(H\left(\left(\pi_{1} f\right)_{n i l}(a)\right) \otimes T H\left(\left(\pi_{1} g\right)_{n i l}(x)\right)\right) .
\end{aligned}
$$

This last equality is a consequence of Definition 2.2.1 (7) and Proposition 2.3.2.

Finally (1)-(6) in Definition 4.5.1 follow from Lemma 4.2.2 (1)-(10) and the elementary properties of the Hopf invariant for tracks in $[9,3.6]$.

Let $\hat{\tau}_{n, m}: \tau_{n, m} \Rightarrow(\cdot)_{n+m}^{(-1)^{n m}}$ be a track between maps

$$
(\cdot)_{n+m}^{(-1)^{n m}}, \tau_{n, m}: S^{n+m} \rightarrow S^{n+m} .
$$

This is a lift of the shuffle permutation in Eq. 18 to the symmetric track group Sym $_{\square}(n+m)$. Given maps $f: \Sigma A \rightarrow \Sigma B, g: \Sigma X \rightarrow \Sigma Y$, we define the following Hopf invariants

$$
\begin{aligned}
& \left.L_{1,1}(f, g)=\bar{\sigma} \operatorname{Hopf}\left(\left(\Sigma^{2} \tau_{\wedge}\right)\left(\mathbb{H}_{g, f}^{\#}\right)^{\boxminus} \hat{\tau}_{1,1}\right) \square\left(\left(\hat{\tau}_{1,1}^{\boxminus} \wedge B \wedge Y\right) \mathbb{H}_{f, g}^{\#}\right)\right), \\
& \left.L_{n, m}(f, g)=\operatorname{Hopf}\left(\left(\Sigma^{m+n} \tau_{\wedge}\right)\left(\mathbb{H}_{m, g, n, f}^{\#}\right)^{\boxminus} \hat{\tau}_{n, m}\right) \square\left(\left(\hat{\tau}_{n, m}^{\boxminus} \wedge B \wedge Y\right) \mathbb{H}_{n, f, m, g}^{\#}\right)\right) .
\end{aligned}
$$

Here $n, m \geq 1$ and $n>1$ or $m>1$, and $\tau_{\wedge}: Y \wedge B \rightarrow B \wedge Y$ is the symmetry map for the smash product. Notice that $L_{n, m}(f, g)$ is a homomorphism, $n, m \geq 1$,

$$
L_{n, m}(f, g): \mathbb{Z}[A] \otimes \mathbb{Z}[X] \longrightarrow \hat{\otimes}^{2}(\mathbb{Z}[B] \otimes \mathbb{Z}[Y]) .
$$

Proposition 4.5.5 For any $n, m \geq 1$ the homomorphism $L_{n, m}(f, g)$ defined above does not depend on the choice of $\hat{\tau}_{n, m}$. Moreover, it only depends on $\left(\pi_{1} f\right)_{\text {nil }}$ and $\left(\pi_{1} g\right)_{n i l}$. Furthermore, $L_{n, m} \in \mathrm{G}\left(\hat{\otimes}^{2}\right)$. In addition,

$$
\mathrm{G}(q)\left(L_{n, m}\right)=\mathrm{G}(q)\left(-\bar{\sigma}\left(\begin{array}{c}
(-1)^{n m} \\
2
\end{array}\right) H(\underline{\#})\right) .
$$

The proof of this proposition is analogous to Proposition 4.5.4. 
We would like to omit $\mathrm{G}(q)$ in the equations of Propositions 4.5.4 and 4.5.5. For this we prove the following two lemmas.

Lemma 4.5.6 If $\Phi: \mathbf{A b} \rightarrow \mathbf{A b}$ is an additive functor which preserves arbitrary filtered colimits then there is an isomorphism

$$
\mathrm{G}(\Phi) \cong \Phi(\mathbb{Z}) .
$$

Proof We claim that the isomorphism sends $\chi$ to $\chi(-1,-1) \in \Phi(\mathbb{Z})$, where -1 is the homomorphism $-1: \mathbb{Z} \rightarrow \mathbb{Z}$. This will be a consequence of the following formula, that we claim to hold. We first notice that

$$
\Phi(\mathbb{Z}[B] \otimes \mathbb{Z}[Y])=\Phi(\mathbb{Z}) \otimes \mathbb{Z}[B] \otimes \mathbb{Z}[Y] .
$$

Given $f:\langle A\rangle_{\text {nil }} \rightarrow\langle B\rangle_{\text {nil }}, g:\langle X\rangle_{\text {nil }} \rightarrow\langle Y\rangle_{\text {nil }}, a \in A$ and $x \in X$, if $f_{a b}(a)=\sum_{i} n_{i} b_{i}$, $g_{a b}(x)=\sum_{j} m_{j} y_{j}$ are the linear expansions with $b_{i} \in B, y_{j} \in Y, n_{i}, m_{j} \in \mathbb{Z}$, then

$$
\chi(f, g)(a \otimes x)=\sum_{i, j} \epsilon\left(n_{i}, m_{j}\right)\left|n_{i} m_{j}\right| \chi(-1,-1) \otimes b_{i} \otimes y_{j} .
$$

Here $\epsilon\left(n_{i}, m_{j}\right)=1$ provided $n_{i}, m_{j}<0$ and it is zero otherwise. Conversely any $\chi$ defined by formula (a) with $\chi(-1,-1)$ arbitrarily chosen out of $\Phi(\mathbb{Z})$ defines an element in $\mathrm{G}(\Phi)$. We will just prove the first part of the claim, the converse is easy.

By (3), (4), (5) and (6) in Definition 4.5.1 we have

$$
\chi(f, g)(a \otimes x)=\sum_{i, j} \chi\left(n_{i}, m_{j}\right) \otimes b_{i} \otimes y_{j} .
$$

for $n_{i}, m_{j}: \mathbb{Z} \rightarrow \mathbb{Z}$. For any $n \in \mathbb{Z}$ we consider the homomorphism $\mu_{n}: \mathbb{Z} \rightarrow$ $\left\langle c_{1}, \ldots, c_{|n|}\right\rangle_{n i l}$ defined by $\mu_{n}(1)=c_{1}+\cdots+c_{|n|}$ if $n>0, \mu_{n}(1)=-c_{1}-\cdots-c_{|n|}$ if $n<0$, and $\mu_{n}(1)=0$ if $n=0$. By Eq. b given $n, m \in \mathbb{Z}$ we have that

$$
\chi\left(\mu_{n}, \mu_{m}\right)=\sum_{i=1}^{|n|} \sum_{j=1}^{|m|} \chi(\epsilon(n), \epsilon(m)) \otimes c_{i} \otimes c_{j} .
$$

Here $\epsilon$ sends a positive integer to 1 , a negative integer to -1 , and zero to itself. Now, again by Definition 4.5.1 (6), we have that $\chi(n, m)$ is the sum of all coefficients in Eq. c, i.e.

$$
\chi(n, m)=|n m| \chi(\epsilon(n), \epsilon(m)) \in \Phi(\mathbb{Z}) .
$$

But by Definition 4.5.1 (1) and (2) the element $\chi(\epsilon(n), \epsilon(m)) \in \Phi(\mathbb{Z})$ is zero unless $n, m<0$, hence we are done.

Lemma 4.5.7 There is a commutative diagram

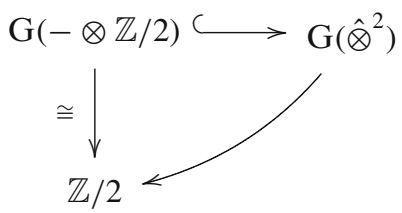


Proof Both vertical homomorphisms send an element $\chi$ in the corresponding G-group to

$$
\chi(-1,-1) \in \mathbb{Z} \otimes \mathbb{Z} / 2=\hat{\otimes}^{2} \mathbb{Z}=\mathbb{Z} / 2 .
$$

The isomorphism is proved in the previous lemma.

The following theorem is a key step towards the proof of Theorem 3.2.1. It is connected to relation (7) in the definition of the tensor product for square groups, see Definition 2.2.1.

Theorem 4.5.8 $K=\bar{\sigma} \tau_{\otimes}\left(1 \otimes \tau_{\otimes} \otimes 1\right)(H \otimes T H) \in \mathrm{G}\left(\hat{\otimes}^{2}\right)$.

Proof By Proposition 4.5.4 and Lemma 4.5.7 we only have to check that

$$
\bar{\sigma} \tau_{\otimes}\left(1 \otimes \tau_{\otimes} \otimes 1\right)(H(-1) \otimes T H(-1))=K(v, v)
$$

for $v: S^{1} \rightarrow S^{1}: z \mapsto z^{-1}$ the complex inversion. It is easy to see from the very definition of $H$ that

$$
\bar{\sigma} \tau_{\otimes}\left(1 \otimes \tau_{\otimes} \otimes 1\right)(H(-1) \otimes T H(-1))=1 \in \mathbb{Z} / 2,
$$

hence this theorem follows from Lemma 4.6.2 below.

The next theorem is the main step in the proof of the commutativity rule for the smash product operation in Theorem 3.2.3.

Theorem 4.5.9 $L_{n, m}=-\bar{\sigma}\left(\begin{array}{c}(-1)^{n m} \\ 2\end{array}\right) H(\#) \in \mathrm{G}\left(\hat{\otimes}^{2}\right), n, m \geq 1$.

Proof By Proposition 4.5.5 and Lemma 4.5.7 we only have to check that

$$
-\bar{\sigma}\left(\begin{array}{c}
(-1)^{n m} \\
2
\end{array}\right) H((-1) \#(-1))=L(v, v)
$$

for $v: S^{1} \rightarrow S^{1}: z \mapsto z^{-1}$ the complex inversion. But $(-1) \#(-1)=1$ and $H(1)=$ $\left(\begin{array}{l}1 \\ 2\end{array}\right)=0$, hence this theorem follows from Lemma 4.6.3 below.

\subsection{Hopf Invariants of Tracks between Orthogonal Transformations}

In this section we are concerned with the computation of Hopf invariants for tracks between self maps of spheres $S^{n}, n \geq 2$. More concretely, we are interested in tracks between maps $A: S^{n} \rightarrow S^{n}$ which are induced by the left action of the orthogonal group $O(n)$ on $S^{n}$, i.e. $A \in O(n)$. The pull-back of this action along the inclusion $\operatorname{Sym}(n) \subset O(n)$, induced by permutation of coordinates in $\mathbb{R}^{n}$, yields the action of 
$S^{n}$ already considered in Section 3.1. Let det: $O(n) \rightarrow\{ \pm 1\}$ be the determinant homomorphism. We consider the group $\{ \pm 1\}$ embedded in $O(n)$ as

$$
\left(\begin{array}{llll}
1 & & & 0 \\
& \ddots & & \\
& & 1 & \\
0 & & & \pm 1
\end{array}\right)
$$

In $[9,6]$ we compute the group $\widetilde{O}(n)$ of tracks $A \Rightarrow \operatorname{det} A, A \in O(n)$, with multiplication given by horizontal composition. Notice that tracks $A \Rightarrow B$ between maps $A, B: S^{n} \rightarrow S^{n}$ with $A, B \in O(n)$ coincide with homotopy classes of paths from $A$ to $B$ in the Lie group $O(n)$, since the $J$-homomorphism $\pi_{1} O(n) \cong \pi_{n+1} S^{n}$ is an isomorphism for $n \geq 2$. We can identify the group $\widetilde{O}(2)$ with the semidirect product $\{ \pm 1\} \ltimes \mathbb{R}$. Here $\mathbb{R}$ is the additive group of real numbers and $\{ \pm 1\}$ is the multiplicative group of order 2 acting on $\mathbb{R}$ by multiplication. See [10, 6.12].

In order to describe $\widetilde{O}(n)$ for $n \geq 3$ we need to recall the definition of the positive Clifford algebra.

Definition 4.6.1 The positive Clifford algebra $C_{+}(n)$ is the unital $\mathbb{R}$-algebra generated by $e_{i}, 1 \leq i \leq n$, with relations

(1) $e_{i}^{2}=1$ for $1 \leq i \leq n$,

(2) $e_{i} e_{j}=-e_{j} e_{i}$ for $1 \leq i<j \leq n$.

Clifford algebras are defined for arbitrary quadratic forms on finite-dimensional vector spaces, see for instance [14, 6.1]. The Clifford algebra defined above corresponds to the quadratic form of the standard positive-definite scalar product in $\mathbb{R}^{n}$. We identify the sphere $S^{n-1}$ with the vectors of Euclidean norm 1 in the vector subspace $\mathbb{R}^{n} \subset C_{+}(n)$ spanned by the generators $e_{i}$. The vectors in $S^{n-1}$ are units in $C_{+}(n)$. Indeed for any $v \in S^{n-1}$ the square $v^{2}=1$ is the unit element in $C_{+}(n)$, so that $v^{-1}=v$. The group $\widetilde{O}(n)$ can be identified with the subgroup of units in $C_{+}(n)$ generated by $S^{n-1}$. This group is also known as the positive pin group.

The group $\widetilde{O}(n)$ is a covering Lie group of $O(n)$ with simply connected components, and with kernel $\mathbb{Z} \subset \mathbb{R}$ if $n=2$ and $\{ \pm 1\}$ if $n \geq 3$. The covering homomorphism

$$
q: \widetilde{O}(n) \longrightarrow O(n)
$$

is defined for $n=2$ as

$$
q(x, y)=\left(\begin{array}{cc}
\cos 2 \pi y & -\sin 2 \pi y \\
x \sin 2 \pi y & x \cos 2 \pi y
\end{array}\right)
$$

For $n \geq 3$ the homomorphism $q$ sends an element $v \in S^{n-1} \subset \widetilde{O}(n) \subset C_{+}(n)$ to the matrix of the reflexion along the plane orthogonal to the unit vector $v$. An element $x \in \widetilde{O}(n)$ is identified with the track $q(x) \Rightarrow \operatorname{det} q(x)$ determined by the push-forward 
along $q$ of the unique track in $\widetilde{O}(n)$ from the point $x$ to the point $e_{n}^{\left(\frac{\operatorname{det} q(x)}{2}\right)}$ if $n \geq 3$, or from $x$ to $(\operatorname{det} q(x), 0)$ if $n=2$. With the track approach the covering map $q$ sends a track to the source map. Moreover, a track $\alpha: 1_{S^{n}} \Rightarrow 1_{S^{n}}$ in the kernel of $q$ is identified with $(1,-\operatorname{Hopf}(\alpha))$ for $n=2$, compare [9, 3.4], and with $\operatorname{Hopf}(\alpha) \in \mathbb{Z} / 2 \cong\{ \pm 1\}$ for $n \geq 3$.

The suspension of tracks defines group inclusions

$$
\Sigma: \widetilde{O}(n) \hookrightarrow \widetilde{O}(n+1) .
$$

For $n \geq 3$ this is induced by the algebra inclusion $C_{+}(n) \hookrightarrow C_{+}(n+1)$ defined by $e_{j} \mapsto e_{j+1}, 1 \leq j \leq n$. For $n=2$ it is given by

$$
(x, y) \mapsto e_{3}^{\left(\begin{array}{c}
x+1 \\
2
\end{array}\right)}\left((\sin \pi y) e_{2}+(\cos \pi y) e_{3}\right) .
$$

For $K$ in Eq. 38 and $v: S^{1} \rightarrow S^{1}: z \mapsto z^{-1}$ we have the following result.

Lemma 4.6.2 $K(v, v)=1$.

Proof We are going to prove the following stronger equality:

$$
\operatorname{Hopf}\left(\left(\mathbb{H}_{v, v}^{\#}\right)^{\boxminus} \square \mathbb{H}_{\nu, v}^{\#}\right)=1 .
$$

From the very definition of $\mathbb{H}$ we obtain the following identity in $\widetilde{O}(2)$.

$$
\mathbb{H}\left(v \wedge S^{1}\right)=\left(1, \frac{1}{4}\right)
$$

Since $\left(v \wedge S^{1}\right) \mathbb{H}$ is the inverse of $\mathbb{H}\left(v \wedge S^{1}\right)$ then

$$
\left(v \wedge S^{1}\right) \mathbb{H}=\left(1,-\frac{1}{4}\right) .
$$

Obviously the identity track $0_{\Sigma v}^{\square}: \Sigma v \Rightarrow \Sigma v$ is $(-1,0)$ in $\widetilde{O}(2)$. By using these equalities we obtain

$$
\begin{aligned}
\left(\mathbb{H}_{v, v}^{\#}\right)^{\boxminus} & =0_{\Sigma v}^{\square}\left(\mathbb{H}\left(v \wedge S^{1}\right)\right) 0_{\Sigma v}^{\square}\left(\left(v \wedge S^{1}\right) \mathbb{H}\right) \\
& =(-1,0)\left(1, \frac{1}{4}\right)(-1,0)\left(1,-\frac{1}{4}\right) \\
& =\left(-1, \frac{1}{4}\right)\left(-1,-\frac{1}{4}\right) \\
& =\left((-1)(-1),-\frac{1}{4}-\frac{1}{4}\right) \\
& =\left(1,-\frac{1}{2}\right),
\end{aligned}
$$




$$
\begin{aligned}
\left(\mathbb{H}_{\bar{v}, v}^{\#}\right)^{\boxminus} & =\left(\mathbb{H}\left(v \wedge S^{1}\right)\right) 0_{\Sigma v}^{\square}\left(\left(v \wedge S^{1}\right) \mathbb{H}\right) 0_{\Sigma v}^{\square} \\
& =\left(1, \frac{1}{4}\right)(-1,0)\left(1,-\frac{1}{4}\right)(-1,0) \\
& =\left(-1,-\frac{1}{4}\right)\left(-1, \frac{1}{4}\right) \\
& =\left((-1)(-1), \frac{1}{4}+\frac{1}{4}\right) \\
& =\left(1, \frac{1}{2}\right) .
\end{aligned}
$$

Let $\beta: 1_{S^{2}} \Rightarrow 1_{S^{2}}$ be a track with $\operatorname{Hopf}(\beta)=1$, so that $\beta=(1,-1)$ in $\widetilde{O}(2)$. Equation a is equivalent to the following equation in $\widetilde{O}(2)$,

$$
\left(\mathbb{H}_{v, v}^{\#}\right)^{\boxminus}=\left(\mathbb{H}_{v, v}^{\#}\right)^{\boxminus} \beta,
$$

which follows from the equalities above.

For $L_{n, m}$ in Eq. 39 we have the following result.

Lemma 4.6.3 $L_{n, m}(v, v)=0, n, m \geq 1$.

Proof We showed in the proof of Lemma 4.6.2 that in $\widetilde{O}(2)$

$$
\begin{aligned}
& \left(\mathbb{H}_{v, v}^{\#}\right)^{\boxminus}=\left(1,-\frac{1}{2}\right), \\
& \left(\mathbb{H}_{v, v}^{\#}\right)^{\boxminus}=\left(1, \frac{1}{2}\right) .
\end{aligned}
$$

For $n=m=1$ we can take $\hat{\tau}_{1,1}=\left(-1,-\frac{1}{4}\right)$. Then in $\widetilde{O}(2)$

$$
\begin{aligned}
\hat{\tau}_{1,1}\left(\mathbb{H}_{v, v}^{\#}\right)^{\boxminus} & =\left(-1,-\frac{1}{4}\right)\left(1,-\frac{1}{2}\right) \\
& =\left(-1,-\frac{1}{4}-\frac{1}{2}\right) \\
& =\left(-1,-\frac{3}{4}\right), \\
\left(\mathbb{H}_{v, v}^{\#}\right)^{\boxminus} \hat{\tau}_{1,1} & =\left(1, \frac{1}{2}\right)\left(-1,-\frac{1}{4}\right) \\
& =\left(-1,-\frac{1}{2}-\frac{1}{4}\right) \\
& =\left(-1,-\frac{3}{4}\right) .
\end{aligned}
$$

This shows that $L_{1,1}(v, v)=0$. 
Suppose now that $n>1$ or $m>1$. By using formula Eq. 40 one can easily check that for any $k \geq 1$

$$
\begin{aligned}
& \Sigma^{k}\left(\mathbb{H}_{v, v}^{\#}\right)^{\boxminus}=e_{k-1} e_{k}, \\
& \Sigma^{k}\left(\mathbb{H}_{v, v}^{\#}\right)^{\boxminus}=e_{k} e_{k-1},
\end{aligned}
$$

in $\widetilde{O}(k+2)$.

For any $k \geq 1$ the shuffle permutation $\tau_{1, k-1}$ can be decomposed as

$$
\tau_{1, k-1}=(k k-1) \cdots(21),
$$

hence for fixed $p, q \geq 0$ with $p+k+q>2$ we can lift $S^{p} \wedge \tau_{1, k-1} \wedge S^{q}$ to $\widetilde{O}(p+k+q)$ by

$$
\hat{\tau}_{1, k-1}=\frac{1}{2^{\frac{k-1}{2}}}\left(e_{p+k}-e_{p+k-1}\right) \cdots\left(e_{p+2}-e_{p+1}\right),
$$

and its inverse in $\widetilde{O}(p+k+q)$ is

$$
\hat{\tau}_{k-1,1}=\frac{1}{2^{\frac{k-1}{2}}}\left(e_{p+2}-e_{p+1}\right) \cdots\left(e_{p+k}-e_{p+k-1}\right) .
$$

By using these equalities we obtain

$$
\begin{aligned}
\left(\mathbb{H}_{n, v, m, v}^{\#}\right)^{\boxminus}= & \left(S^{n-1} \wedge \tau_{m-1,1} \wedge S^{1}\right)\left(\Sigma^{m+n-2}\left(\mathbb{H}_{v, v}^{\#}\right)^{\boxminus}\right)\left(S^{n-1} \wedge \tau_{1, m-1} \wedge S^{1}\right) \\
= & \hat{\tau}_{m-1,1}\left(\Sigma^{m+n-2}\left(\mathbb{H}_{v, v}^{\#}\right)^{\boxminus}\right) \hat{\tau}_{1, m-1} \\
= & \frac{1}{2^{m-1}}\left(e_{n+1}-e_{n}\right) \cdots\left(e_{n+m-1}-e_{n+m-2}\right) e_{n+m-1} e_{n+m} \\
& \cdot\left(e_{n+m-1}-e_{n+m-2}\right) \cdots\left(e_{n+1}-e_{n}\right), \\
\left(\mathbb{H}_{\frac{m}{m, v, n, v}}^{\#}=\right. & \left(S^{m-1} \wedge \tau_{n-1,1} \wedge S^{1}\right)\left(\Sigma^{n+n-2}\left(\mathbb{H}_{v, v}^{\#}\right)^{\boxminus}\right)\left(S^{m-1} \wedge \tau_{1, n-1} \wedge S^{1}\right) \\
= & \hat{\tau}_{n-1,1}\left(\Sigma^{n+m-2}\left(\mathbb{H}_{v, v}^{\#}\right)^{\boxminus}\right) \hat{\tau}_{1, n-1} \\
= & \frac{1}{2^{n-1}}\left(e_{m+1}-e_{m}\right) \cdots\left(e_{n+m-1}-e_{n+m-2}\right) e_{n+m} e_{n+m-1} \\
& \cdot\left(e_{n+m-1}-e_{n+m-2}\right) \cdots\left(e_{m+1}-e_{m}\right) .
\end{aligned}
$$

For $j>i$ we have the following useful identities.

$$
\begin{aligned}
e_{j}\left(e_{j}-e_{i}\right) & =-\left(e_{j}-e_{i}\right) e_{i}, \\
e_{i}\left(e_{j}-e_{i}\right) & =-\left(e_{j}-e_{i}\right) e_{j}, \\
\left(e_{j}-e_{i}\right)^{2} & =2 .
\end{aligned}
$$

By using Eqs. a, b, c and the formulas above one can easily check that

$$
\begin{aligned}
& \left(\mathbb{H}_{n, v, m, v}^{\#}\right)^{\boxminus}=e_{n} e_{n+m}, \\
& \left(\mathbb{H}_{m, v, n, v}^{\#}\right)^{\boxminus}=e_{n+m} e_{m} .
\end{aligned}
$$

The identity $\tau_{n, m}=\tau_{1, n+m-1}^{n}$ holds, therefore we can also take $\hat{\tau}_{n, m}=\hat{\tau}_{1, n+m-1}^{n}$. With this choice it is not difficult to compute that

$$
\hat{\tau}_{n, m}\left(\mathbb{H}_{n, v, m, v}^{\#}\right)^{\boxminus}=\left(\mathbb{H}_{m, v, n, v}^{\#}\right)^{\boxminus} \hat{\tau}_{n, m}
$$


in $\widetilde{O}(n+m)$. For this one uses Eqs. a and b. This last equation is equivalent to $L_{n, m}(v, v)=0$.

\subsection{Properties of the Smash Product in Dimensions $\geq 1$}

Restricting to dimensions $\geq 1$, in this section we show a series of properties of the smash product operation for secondary homotopy groups in Definition 4.3.1 which imply Theorems 3.2.1, 3.2.2, 3.2.3 and 3.3.10 within this range. The case of dimension 0 is a consequence of the fact that secondary homotopy groups are track functors and of the first technical lemma in the next section.

In this section we will work with the track category Top*. This track category has a strict zero object 0 so that the zero morphism $0: X \rightarrow Y$ is always defined for a pair of pointed spaces. In this situation the golden rule says that

The composition of a trivial map 0 with any track $F$ is always a trivial track $0 F=0^{\square}, F 0=0^{\square}$.

This is an obvious but crucial property that will be very useful for computations.

Let $n, m \geq 1$ and $0 \leq i, j, i+j \leq 1$.

We need to show that the square group morphisms Eq. 36 are well defined. There is nothing to check in case $i=j=0$. For $i+j=1$ we define operations

$$
\wedge, \wedge: \pi_{n, i} X \times \pi_{m, j} Y \longrightarrow \Pi_{n+m, 1}(X \wedge Y)
$$

as in Definition 4.3.1. For this we need to choose $\bar{g}$ more carefully in certain cases. For example, in order to define $g \wedge[f, F]$ and $g \wedge[f, F]$ when $n=1$ and $i=0$ we need to take $\bar{g}$ in such a way that $\left(\pi_{1} \bar{g}\right)(1)=g \in\langle\Omega X\rangle$.

It is not completely immediate that the operations $\wedge$ and $\Lambda$ do not depend on choices. We check here for instance that $g \wedge[f, F]$ does not depend on the choice of $\bar{g}, f$ and $F$. Let $\bar{g}^{\prime}, f^{\prime}$ and $F^{\prime}$ be another choice. Then there are tracks $N_{1}: \Sigma^{n-1} \bar{g}^{\prime} \Rightarrow \Sigma^{n-1} \bar{g}$ and $N_{2}: \Sigma^{m-1} f^{\prime} \Rightarrow \Sigma^{m-1} f$ with trivial Hopf invariant such that $F^{\prime}=F \square\left(\right.$ ev $\left.N_{2}\right)$. One can now use Lemmas 4.4.1, 4.2.3, and 4.4.2 together with the golden rule to show that, up to a permutation in the spherical coordinates, the track $\Sigma\left(N_{1} \# N_{2}\right): \Sigma^{n+m-1}\left(\bar{g}^{\prime} \# f^{\prime}\right) \Rightarrow \Sigma^{n+m-1}(\bar{g} \# f)$ is a track with trivial Hopf invariant which determines the desired equality.

In the next lemma we establish the fundamental properties of Eq. 41.

\section{Lemma 4.7.1}

(1) The operation $\wedge$ is left linear

$$
\begin{aligned}
(x+y) \wedge[f, F] & =x \wedge[f, F]+y \wedge[f, F], \\
([f, F]+[g, G]) \wedge x & =[f, F] \wedge x+[g, G] \wedge x .
\end{aligned}
$$

(2) The operation $\wedge$ is right linear

$$
\begin{aligned}
{[f, F] \wedge(x+y) } & =[f, F] \underline{\wedge}+[f, F] \underline{\wedge y,} \\
x \underline{\wedge}([f, F]+[g, G]) & =x \underline{\wedge}[f, F]+x \underline{\wedge}[g, G] .
\end{aligned}
$$


(3) For $m \geq 2, i=0$ and $j=1$, given $a \in \otimes^{2} \mathbb{Z}\left[\Omega^{m} Y\right]$ the equality $x \wedge P(a)=$ $P\left(\otimes{ }^{2} \mathbb{Z}[\wedge]\right)\left(1 \otimes \tau_{\otimes} \otimes 1\right)(\Delta(x) \otimes a)$ holds.

(4) For $m \geq 2, i=1$ and $j=0$, given $a \in \otimes^{2} \mathbb{Z}\left[\Omega^{m} Y\right]$ the equality $[f, F] \wedge \partial P(a)=$ $P\left(\otimes{ }^{2} \mathbb{Z}[\wedge]\right)\left(1 \otimes \tau_{\otimes} \otimes 1\right)(\Delta \partial[f, F] \otimes a)$ holds.

(5) For $n \geq 2$, $i=1$ and $j=0$, given $a \in \otimes^{2} \mathbb{Z}\left[\Omega^{n} X\right]$ the equality $P(a) \wedge x=$ $P\left(\otimes^{2} \mathbb{Z}[\wedge]\right)\left(1 \otimes \tau_{\otimes} \otimes 1\right)(a \otimes \Delta(x))$ holds.

(6) For $n \geq 2, i=0$ and $j=1$, given $a \in \otimes^{2} \mathbb{Z}\left[\Omega^{n} X\right]$ the following equality holds

$$
\partial P(a) \wedge[f, F]=P\left(\otimes^{2} \mathbb{Z}[\wedge]\right)\left(1 \otimes \tau_{\otimes} \otimes 1\right)(a \otimes \Delta \partial[f, F]) .
$$

(7) If $m=1, i=0$ and $j=1$, the following equality holds

$$
g \wedge\left(-[f, F]+[f, F]^{x}\right)=P\left(\otimes^{2} \mathbb{Z}[\wedge]\right)\left(1 \otimes \tau_{\otimes} \otimes 1\right)(\Delta(g) \otimes\{x\} \otimes\{\partial[f, F]\}) .
$$

(8) For $n=1, i=1$ and $j=0$, the following equality holds

$$
\left(-[f, F]+[f, F]^{x}\right) \wedge g=P\left(\otimes^{2} \mathbb{Z}[\wedge]\right)\left(1 \otimes \tau_{\otimes} \otimes 1\right)(\{x\} \otimes\{\partial[f, F]\} \otimes \Delta(g)) .
$$

(9) The equality $g \wedge[f, F]-g \wedge[f, F]=P\left(\otimes^{2} \mathbb{Z}[\wedge]\right)\left(1 \otimes \tau_{\otimes} \otimes 1\right)(H(g) \otimes T H \partial[f, F])$ holds.

(10) The equality $[f, F] \wedge g-[f, F] \wedge g=P\left(\otimes^{2} \mathbb{Z}[\wedge]\right)\left(1 \otimes \tau_{\otimes} \otimes 1\right)(H \partial[f, F] \otimes T H(g))$ holds.

(11) The equalities $(\partial[f, F]) \wedge[g, G]=[f, F] \wedge(\partial[g, G])$ and $(\partial[f, F]) \wedge[g, G]=$ $[f, F] \wedge(\partial[g, G])$ hold.

Proof Let us check the first equation in (1).

$$
\begin{aligned}
x \wedge[f, F]+y \wedge[f, F]= & (\Sigma \wedge)(\bar{x} \# f, \bar{y} \# f) \mu, \\
& \left(\left(\bar{x}_{e v} \wedge f\right) \square\left(e v\left(\Sigma^{n+m} \wedge\right) \mathbb{H}_{n, \bar{x}, m, f}^{\#}\right),\right. \\
& \left.\left.\left(\bar{y}_{e v} \wedge f\right) \square\left(\operatorname{ev}\left(\Sigma^{n+m} \wedge\right) \mathbb{H}_{n, \bar{y}, m, f}^{\#}\right)\right) \mu\right] \\
4.1 .1(3) \text { and }(\mathrm{GR})= & ((\Sigma \wedge)((\bar{x}, \bar{y}) \# f) \mu, \\
& \left(\left(\bar{x}_{e v} \wedge f\right) \square\left(e v\left(\Sigma^{n+m} \wedge\right) \mathbb{H}_{n, \bar{x}, m, f}^{\#}\right),\right. \\
& \left.\left.\left(\bar{y}_{e v} \wedge f\right) \square\left(e v\left(\Sigma^{n+m} \wedge\right) \mathbb{H}_{n, \bar{y}, m, f}^{\#}\right)\right) \mathbb{H}_{n, \mu, m, 1_{S^{1}}}^{\#}\right] \\
4.1 .1(7)= & ((\Sigma \wedge)(((\bar{x}, \bar{y}) \mu) \# f), \\
& \left(\left(\bar{x}_{e v} \wedge F, \bar{y}_{e v} \wedge f\right)\left(\Sigma^{n-1} \mu \wedge S^{m}\right)\right) \square \\
& \left.\left(e v\left(\Sigma^{n+m} \wedge\right)\left(\mathbb{H}_{n, \bar{x}, m, f}^{\#}, \mathbb{H}_{n, \bar{y}, m, f}^{\#}\right) \mathbb{H}_{n, \mu, m, 1_{S^{1}}}^{\#}\right)\right] \\
4.2 .2(3) \text { and }(7)= & ((\Sigma \wedge)(((\bar{x}, \bar{y}) \mu) \# f), \\
& \left(((\bar{x}, \bar{y}) \mu)_{e v} \wedge f\right) \square\left(e v\left(\Sigma^{n+m} \wedge\right) \mathbb{H}_{n,(\bar{x}, \bar{y}) \mu, m, f}^{\#}\right] \\
= & (x+y) \wedge[f, F] .
\end{aligned}
$$

The second equation in (1) and the equations in (2) are analogous. 
In order to check equation (3) we notice that $x \wedge P(a)$ is represented by the following diagram

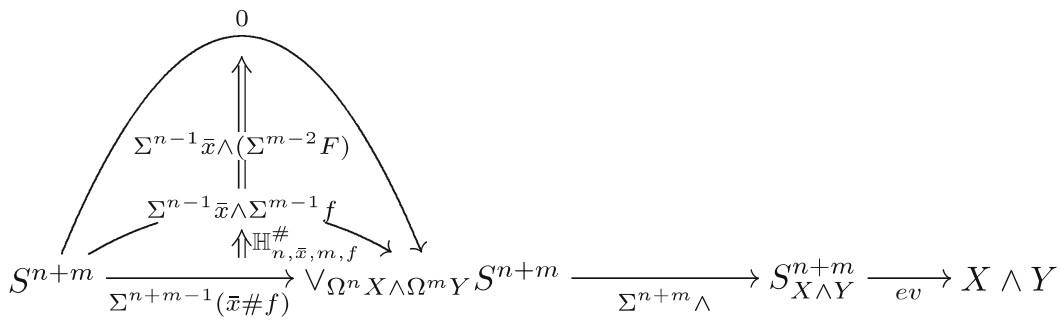

where $F: \Sigma f \Rightarrow 0$ is any track with $\operatorname{Hopf}(F)=-\tau_{\otimes}(a)$. Here we use claim (*) in the proof of $[9,4.9]$. By Lemmas 4.2.3 (1), 4.2.2 (2) and the golden rule this diagram coincides with

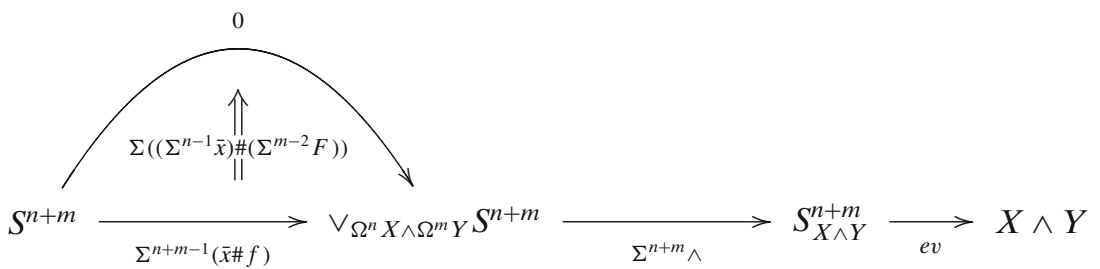

Now one can use Lemmas 4.4.1 (2) and 4.4.2 to check that

$$
\operatorname{Hopf}\left(\Sigma\left(\left(\Sigma^{n-1} \bar{x}\right) \#\left(\Sigma^{m-2} F\right)\right)\right)=-\bar{\sigma} \tau_{\otimes}\left(1 \otimes \tau_{\otimes} \otimes 1\right)(\Delta(x) \otimes a)
$$

hence (3) follows form claim (*) in the proof of [9, 4.9]. Equation (5) is analogous. Equations (7) and (8) are unstable versions of (3) and (5). We leave them to the reader.

Both sides of the first equation in (11) are represented by

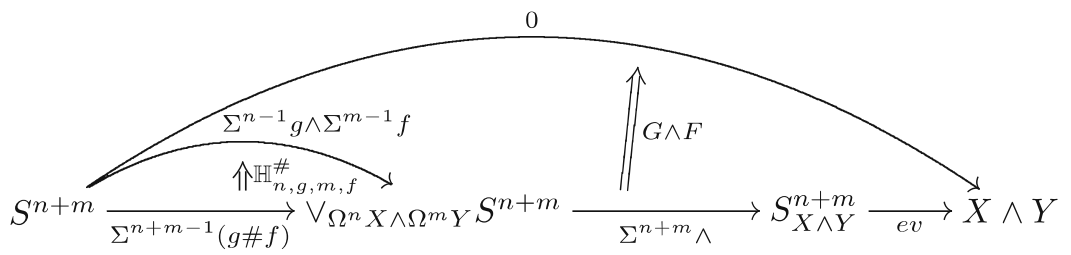

In order to check this fact one only needs to use the golden rule. Similarly for the second equation in (11). Now (4) and (6) follow from (3), (5) and (11).

Finally (9) and (10) follow from Theorem 4.5.8, Lemma 4.4.2, and claim (*) in the proof of $[9,4.9]$.

The equalities in Lemma 4.7.1 can be used to check the following properties of the operations in Eq. 41. 


\section{Lemma 4.7.2}

(1) For $n=1$ and $i=0$ the elements $g \wedge[f, F]$ and $g \wedge[f, F]$ only depend on $g \in$ $\Pi_{1,0} X$.

(2) For $m=1$ and $j=0$ the elements $[f, F] \wedge g$ and $[f, F] \wedge g$ only depend on $g \in$ $\Pi_{1,0} Y$.

(3) For $m=2$ and $j=1$ the elements $g \wedge[f, F]$ and $g \wedge[f, F]$ only depend on $[f, F] \in \Pi_{2,1} X$.

(4) For $n=2$ and $i=1$ the elements $[f, F] \wedge g$ and $[f, F] \wedge g$ only depend on $[f, F] \in$ $\Pi_{2,1} X$.

Lemma 4.7.2 allows us to define the following operations when $m=j=1$ or $n=$ $i=1$.

$$
\begin{aligned}
& \wedge, \wedge: \Pi_{n, 0} X \times\left(\pi_{1,1} Y \times\left(\hat{\otimes}^{2} \mathbb{Z}[\Omega Y]\right)\right) \longrightarrow \Pi_{n+1,1}(X \wedge Y), \\
& \wedge, \underline{\Delta}:\left(\pi_{1,1} X \times\left(\hat{\otimes}^{2} \mathbb{Z}[\Omega X]\right)\right) \times \Pi_{m, 0} Y \longrightarrow \Pi_{1+m, 1}(X \wedge Y),
\end{aligned}
$$

by the formulas

$$
\begin{aligned}
g \wedge([f, F], a) & =g \wedge[f, F]+P\left(\otimes^{2} \mathbb{Z}[\wedge]\right)\left(1 \otimes \tau_{\otimes} \otimes 1\right)(\Delta(g) \otimes a), \\
g \wedge([f, F], a) & =g \wedge[f, F]+P\left(\otimes^{2} \mathbb{Z}[\wedge]\right)\left(1 \otimes \tau_{\otimes} \otimes 1\right)(\{g\} \otimes\{g\} \otimes a), \\
([f, F], a) \wedge g & =[f, F] \wedge g+P\left(\otimes^{2} \mathbb{Z}[\wedge]\right)\left(1 \otimes \tau_{\otimes} \otimes 1\right)(a \otimes\{g\} \otimes\{g\}), \\
([f, F], a) \wedge g & =[f, F] \wedge g+P\left(\otimes^{2} \mathbb{Z}[\wedge]\right)\left(1 \otimes \tau_{\otimes} \otimes 1\right)(a \otimes \Delta(g)) .
\end{aligned}
$$

By using Lemma 4.7.1 one can check the following one.

\section{Lemma 4.7.3}

(1) For $m=j=1$ the elements $g \wedge([f, F], a)$ and $g \wedge([f, F], a)$ only depend on $([f, F], a) \in \Pi_{1,1} Y$

(2) For $n=i=1$ the elements $([f, F], a) \wedge g$ and $([f, F], a) \wedge g$ only depend on $([f, F], a) \in \Pi_{1,1} X$.

Moreover, one can extend all properties (1)-(6) and (9)-(11) in Lemma 4.7.1 to the operations in Eq. 42. The explicit statement is left to the reader.

In order to prove the following Lemma one uses Lemma 4.2.2 (11) and (12). We leave the details as an exercise.

Lemma 4.7.4 The following associativity rules for the operations (41) hold.

(1) $g \wedge\left(g^{\prime} \wedge[f, F]\right)=\left(g \# g^{\prime}\right) \wedge[f, F]$,

(2) $g \wedge\left([f, F] \wedge g^{\prime}\right)=(g \wedge[f, F]) \wedge g^{\prime}$,

(3) $[f, F] \wedge\left(g \# g^{\prime}\right)=([f, F] \wedge g) \wedge g^{\prime}$,

(4) $g \underline{\wedge}\left(g^{\prime} \underline{\wedge}[f, F]\right)=\left(g \underline{\#} g^{\prime}\right) \underline{\wedge}[f, F]$,

(5) $g \wedge\left([f, F] \wedge g^{\prime}\right)=(g \wedge[f, F]) \wedge g^{\prime}$,

(6) $[f, F] \underline{\wedge}\left(g \# g^{\prime}\right)=([f, F] \underline{\wedge} g) \underline{\wedge} g^{\prime}$.

One can accordingly obtain associativity properties involving also the operations in Eq. 42. 
Recall that $\hat{\tau}_{n, m}: \tau_{n, m} \Rightarrow(\cdot)_{n+m}^{(-1)^{n m}} \in \operatorname{Sym}_{\square}(n+m)$ is a track from the shuffle permutation in Eq. 18 to its sign, and $\tau_{\wedge}$ is the symmetry isomorphism for the smash product of pointed spaces. In the following lemma we establish the commutativity rule for the operations in Eq. 41. One can similarly state the commutativity rules for Eq. 42.

Lemma 4.7.5 Given two pointed spaces $X, Y,[f, F] \in \Pi_{n, 1} X$, and $g \in \Pi_{m, 0} Y$ we have the equalities

(1) $\left\langle g \wedge \partial[f, F], \hat{\tau}_{n, m}\right\rangle=-\left(\tau_{\wedge}\right)_{*}([f, F] \wedge g)+\left((-1)^{n m}\right)^{*}(g \wedge[f, F])$,

(2) $\left\langle g \wedge \partial[f, F], \hat{\tau}_{n, m}\right\rangle=-\left(\tau_{\wedge}\right)_{*}([f, F] \wedge g)+\left((-1)^{n m}\right)^{*}(g \wedge[f, F])$.

Moreover if $g \in \Pi_{n, 0} X$ and $[f, F] \in \Pi_{m, 1} Y$

(3) $\left\langle\partial[f, F] \wedge g, \hat{\tau}_{n, m}\right\rangle=-\left(\tau_{\wedge}\right)_{*}(g \wedge[f, F])+\left((-1)^{n m}\right)^{*}([f, F] \wedge g)$,

(4) $\left\langle\partial[f, F] \wedge g, \hat{\tau}_{n, m}\right\rangle=-\left(\tau_{\wedge}\right)_{*}(g \wedge[f, F])+\left((-1)^{n m}\right)^{*}([f, F] \wedge g)$.

Proof Here we prove (1). The other equations are analogous.

The following diagram of pointed sets is commutative.

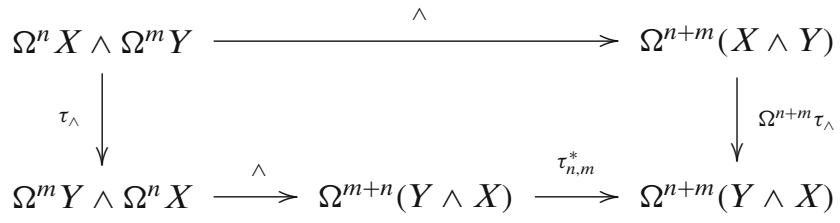

Therefore using the definition of induced morphisms for secondary homotopy groups in $[9,4.2]$ we have

$$
\begin{aligned}
\left(\tau_{\wedge}\right)_{*}([f, F] \wedge g)= & {\left[\left(\Sigma \Omega^{n+m} \tau_{\wedge}\right)(\Sigma \wedge)(f \# \bar{g}),\right.} \\
& \left.\tau_{\wedge}\left(\left(F \wedge \bar{g}_{e v}\right) \square\left(e v\left(\Sigma^{n+m} \wedge\right) \mathbb{H}_{n, f, m, g}^{\#}\right)\right)\right] \\
= & {\left[\left(\Sigma \tau_{n, m}^{*}\right)(\Sigma \wedge)\left(\Sigma \tau_{\wedge}\right)(f \# \bar{g}),\right.} \\
& \left.\left.\left(\tau_{\wedge}\left(F \wedge \bar{g}_{e v}\right)\right) \square\left(\tau_{\wedge} e v\left(\Sigma^{n+m} \wedge\right) \mathbb{H}_{n, f, m, g}^{\#}\right)\right)\right] \\
\text { Eq. } 8= & {\left[\left(\Sigma \tau_{n, m}^{*}\right)(\Sigma \wedge)(\bar{g} \# f),\right.} \\
& \left.\left.\left(\tau_{\wedge}\left(F \wedge \bar{g}_{e v}\right)\right) \square\left(\operatorname{ev}\left(\Sigma^{n+m} \wedge\right)\left(\tau_{n, m} \wedge \tau_{\wedge}\right) \mathbb{H}_{n, f, m, g}^{\#}\right)\right)\right] \\
= & (\mathrm{a}) .
\end{aligned}
$$

On the other hand using claim (*) in the proof of $[9,4.9]$, if

$$
Q:\left(\Sigma^{n+m} \wedge\right)\left((\cdot)_{n+m}^{(-1)^{n m}} \wedge \tau_{\wedge}\right)\left(\Sigma^{n+m-1}(f \# \bar{g})\right) \Rightarrow\left(\Sigma^{n+m} \wedge\right)\left(\Sigma^{n+m-1}(\bar{g} \# f)\right)(\cdot)_{n+m}^{(-1)^{n m}}
$$

is a track with

$$
\overline{\operatorname{Hopf}}(Q)=-\bar{\sigma} \tau_{\otimes}\left(\begin{array}{c}
(-1)^{n m} \\
2
\end{array}\right) H(g \wedge \partial[f, F])
$$


then

$$
\begin{aligned}
& \left((-1)^{n m}\right)^{*}(g \wedge[f, F])=\left[\left((\cdot)^{(-1)^{n m}} \wedge \Omega^{m+n}(Y \wedge X)\right)(\Sigma \wedge)(\bar{g} \# f),\right. \\
& \left(\left(\bar{g}_{e v} \wedge f\right)(\cdot)_{m+n}^{(-1)^{n m}}\right) \\
& \left.\square\left(\operatorname{ev}\left(\Sigma^{m+n} \wedge\right) \mathbb{H}_{m, \bar{g}, n, f}^{\#}(\cdot)_{m+n}^{(-1)^{n m}}\right) \square(e v Q)\right] \\
& (\mathrm{GR})=\left[\left((\cdot)^{(-1)^{n m}} \wedge \Omega^{m+n}(Y \wedge X)\right)(\Sigma \wedge)(\bar{g} \# f),\right. \\
& \left(\left(\bar{g}_{e v} \wedge f\right) \tau_{n, m}\right) \\
& \left.\square\left(\operatorname{ev}\left(\Sigma^{m+n} \wedge\right) \mathbb{H}_{m, \bar{g}, n, f}^{\#} \hat{\tau}_{n, m}^{\boxminus}\right) \square(e v Q)\right] \\
& \text { (Theorem 4.5.9) }=\left[\left((\cdot)^{(-1)^{n m}} \wedge \Omega^{m+n}(Y \wedge X)\right)(\Sigma \wedge)(\bar{g} \# f)\right. \text {, } \\
& \left.\left(\tau_{\wedge}\left(F \wedge \bar{g}_{e v}\right)\right) \square\left(\operatorname{ev}\left(\Sigma^{n+m} \wedge\right)\left(\hat{\tau}_{n, m}^{\boxminus} \wedge \tau_{\wedge}\right) \mathbb{H}_{n, f, m, \bar{g}}^{\#}\right)\right] \\
& =(\mathrm{b}) \text {. }
\end{aligned}
$$

Finally given a map $\varepsilon: S^{1} \rightarrow S^{1} \vee S^{1}$ with $\left(\pi_{1} \varepsilon\right)_{\text {nil }}(1)=-a+b \in\langle a, b\rangle_{\text {nil }}$ and a track $N:(1,1)\left(\Sigma^{n+m-1} \varepsilon\right) \Rightarrow 0$ with $\overline{\operatorname{Hopf}}(N)=0$

$$
\begin{gathered}
-(\mathrm{a})+(\mathrm{b})=\left[\left(\left(\Sigma \tau_{n, m}^{*}\right)(\Sigma \wedge)(\bar{g} \# f),\left((\cdot)^{(-1)^{n m}} \wedge \Omega^{m+n}(Y \wedge X)(\Sigma \wedge)(\bar{g} \# f)\right) \varepsilon,\right.\right. \\
\left(\tau_{\wedge}\left(F \wedge \bar{g}_{e v}, F \wedge \bar{g}_{e v}\right)\left(\Sigma^{n+m} \varepsilon\right)\right) \\
\square\left(e v ( \Sigma ^ { n + m } \wedge ) \left(\left(\tau_{n, m} \wedge \tau_{\wedge}\right) \mathbb{H}_{n, f, m, g}^{\#},\right.\right. \\
\left.\left.\left.\left(\hat{\tau}_{n, m}^{\boxminus} \wedge \tau_{\wedge}\right) \mathbb{H}_{n, f, m, g}^{\#}\right)\left(\Sigma^{n+m} \varepsilon\right)\right)\right] \\
=\left[\left(\left(\Sigma \tau_{n, m}^{*}\right)(\Sigma \wedge)(\bar{g} \# f),\left((\cdot)^{(-1)^{n m}} \wedge \Omega^{m+n}(Y \wedge X)(\Sigma \wedge)(\bar{g} \# f)\right) \varepsilon,\right.\right. \\
\left(\tau_{\wedge}\left(F \wedge \bar{g}_{e v}\right)(1,1)\left(\Sigma^{n+m} \varepsilon\right)\right) \\
\left.\square\left(e v\left(\Sigma^{n+m} \wedge\right)\left(\hat{\tau}_{n, m}^{\boxminus} \wedge \tau_{\wedge}\right) \mathbb{H}_{n, f, m, g}^{\#}\right)(1,1)\left(\Sigma^{n+m} \varepsilon\right)\right) \\
\square\left(e v ( \Sigma ^ { n + m } \wedge ) \left(\left(\hat{\tau}_{n, m} \wedge \tau_{\wedge}\right)\left(\Sigma^{n+m-1}(f \# \bar{g})\right),\right.\right. \\
\left.\left.\left.\left((\cdot)_{n+m}^{(-1)^{n m}} \wedge \tau_{\wedge}\right)\left(\Sigma^{n+m-1}(f \# \bar{g})\right)\right)\left(\Sigma^{n+m} \varepsilon\right)\right)\right]
\end{gathered}
$$

Eq. $8=\left[\left(\left(\Sigma \tau_{n, m}^{*}\right)(\Sigma \wedge)(\bar{g} \# f),\left((\cdot)^{(-1)^{n m}} \wedge \Omega^{m+n}(Y \wedge X)(\Sigma \wedge)(\bar{g} \# f)\right) \varepsilon\right.\right.$,

$$
\begin{gathered}
\left(\left(\left(\tau_{\wedge}\left(F \wedge \bar{g}_{e v}\right)\right)\right.\right. \\
\left.\left.\square\left(e v\left(\Sigma^{n+m} \wedge\right)\left(\hat{\tau}_{n, m}^{\boxminus} \wedge \tau_{\wedge}\right) \mathbb{H}_{n, f, m, g}^{\#}\right)\right)(1,1)\left(\Sigma^{n+m} \varepsilon\right)\right) \\
\square\left(e v ( \Sigma ^ { n + m } \wedge ) \left(\left(\hat{\tau}_{n, m} \wedge \Omega^{m} Y \wedge \Omega^{n} X\right)\left(\Sigma^{n+m-1}(\bar{g} \# f)\right),\right.\right. \\
\left.\left.\left.\left((\cdot)_{n+m}^{(-1)^{n m}} \wedge \Omega^{m} Y \wedge \Omega^{n} X\right)\left(\Sigma^{n+m-1}(\bar{g} \# f)\right)\right)\left(\Sigma^{n+m} \varepsilon\right)\right)\right] \\
(\mathrm{GR})=\left[\left(\left(\Sigma \tau_{n, m}^{*}\right)(\Sigma \wedge)(\bar{g} \# f),\left((\cdot)^{(-1)^{n m}} \wedge \Omega^{m+n}(Y \wedge X)\right)(\Sigma \wedge)(\bar{g} \# f)\right) \varepsilon,\right. \\
\left(\left(\left(\tau_{\wedge}\left(F \wedge \bar{g}_{e v}\right)\right)\right.\right. \\
\left.\left.\square\left(e v\left(\Sigma^{n+m} \wedge\right)\left(\hat{\tau}_{n, m}^{\boxminus} \wedge \tau_{\wedge}\right) \mathbb{H}_{n, f, m, g}^{\#}\right)\right) N\right) \\
\square\left(e v ( \Sigma ^ { n + m } \wedge ) \left(\left(\hat{\tau}_{n, m} \wedge \Omega^{m} Y \wedge \Omega^{n} X\right)\left(\Sigma^{n+m-1}(\bar{g} \# f)\right),\right.\right. \\
\left.\left.\left.\left((\cdot)_{n+m}^{(-1)^{n m}} \wedge \Omega^{m} Y \wedge \Omega^{n} X\right)\left(\Sigma^{n+m-1}(\bar{g} \# f)\right)\right)\left(\Sigma^{n+m} \varepsilon\right)\right)\right]
\end{gathered}
$$




$$
\begin{gathered}
(\mathrm{GR}), \text { Eq. } 8=\left[\left(\left(\Sigma \tau_{n, m}^{*}\right)(\Sigma \wedge)(\bar{g} \# f),\left((\cdot)^{(-1)^{n m}} \wedge \Omega^{m+n}(Y \wedge X)\right)(\Sigma \wedge)(\bar{g} \# f)\right) \varepsilon,\right. \\
\left(e v\left(\Sigma^{n+m} \wedge\right)\left(S^{n+m-1} \wedge(\cdot)^{(-1)^{n m}} \wedge \Omega^{m} Y \wedge \Omega^{n} X\right)\right. \\
\left.\left(\Sigma^{n+m-1}(\bar{g} \# f)\right) N\right) \\
\square\left(e v ( \Sigma ^ { n + m } \wedge ) \left(\left(\hat{\tau}_{n, m} \wedge \Omega^{m} Y \wedge \Omega^{n} X\right)\left(\Sigma^{n+m-1}(\bar{g} \# f)\right),\right.\right. \\
\left.\left.\left.\left((\cdot)_{n+m}^{(-1)^{n m}} \wedge \Omega^{m} Y \wedge \Omega^{n} X\right)\left(\Sigma^{n+m-1}(\bar{g} \# f)\right)\right)\left(\Sigma^{n+m} \varepsilon\right)\right)\right] \\
=\left\langle g \wedge \partial[f, F], \hat{\tau}_{n, m}\right\rangle
\end{gathered}
$$

Here we use the definition of the bracket operation $\langle-,-\rangle$ in $[10,4.5]$.

Finally we prove the compatibility of the smash product operation with the action of the symmetric track group.

Lemma 4.7.6 Let $f \in \Pi_{n, 0} X$ and $g \in \Pi_{m, 0} Y$. Given $\hat{\sigma} \in \operatorname{Sym}_{\square}(m)$ and $\hat{\gamma} \in \operatorname{Sym}_{\square}(n)$ with $\delta(\hat{\sigma})=\sigma$ and $\delta(\hat{\gamma})=\gamma$ the following equalities hold.

$$
\begin{aligned}
& \begin{aligned}
\left\langle f \wedge g, S^{n} \wedge \hat{\sigma}\right\rangle= & f \wedge\langle g, \hat{\sigma}\rangle \\
& +P\left(\otimes^{2} \mathbb{Z}[\wedge]\right)\left(1 \otimes \tau_{\otimes} \otimes 1\right)\left(H(f) \otimes\left(-\sigma^{*} g+(\operatorname{sign} \sigma)^{*} g \mid \sigma^{*} g\right)_{H}\right), \\
\left\langle f \wedge g, \hat{\tau}_{n, m}^{-1}(\right. & \left.\left.S^{m} \wedge \hat{\gamma}\right) \hat{\tau}_{n, m} \omega^{n m}\right\rangle=\langle f, \hat{\gamma}\rangle \wedge g \\
+ & P\left(\otimes^{2} \mathbb{Z}[\wedge]\right)\left(1 \otimes \tau_{\otimes} \otimes 1\right)\left(\left(-\gamma^{*} f+(\operatorname{sign} \gamma)^{*} f \mid \gamma^{*} f\right)_{H} \otimes H(g)\right) .
\end{aligned}
\end{aligned}
$$

Proof Equation (2) follows from (1) and the laws of a sign group action, see [10, Lemma 3.7 and Definition 3.4 (5)]. Let us prove (1). By Definition 4.2.2 (1), (2), (5), (6), (8) and (9), given $\varepsilon: S^{1} \rightarrow S^{1} \vee S^{1}$ with $\left(\pi_{1} \varepsilon\right)_{\text {nil }}(1)=-a+b \in\langle a, b\rangle_{\text {nil }}$

$$
\begin{aligned}
\mathbb{H}_{\left.n, \bar{f}, m,\left(\Sigma \sigma^{*},(\cdot)\right)^{\operatorname{sign} \sigma} \wedge \Omega^{m} Y\right)(\bar{g} \vee \bar{g}) \varepsilon}^{\#}= & \left(\Sigma^{n+m}\left(\Omega^{n} X \wedge \sigma^{*}\right),\right. \\
& \left.(\cdot)_{n+m}^{\operatorname{sign} \sigma} \wedge \Omega^{n} X \wedge \Omega^{m} Y\right) \mathbb{H}_{n, \bar{f}, m,(\bar{g} \vee \bar{g}) \varepsilon}^{\#}, \\
& \left(\mathbb{H}_{n, \bar{f}, m,(\bar{g} \vee \bar{g}) \varepsilon}^{\#}=\left(\mathbb{H}_{n, \bar{f}, m, \bar{g}}^{\#} \vee \mathbb{H}_{n, \bar{f}, m, \bar{g}}^{\#}\right)\left(\Sigma^{n+m-1} \varepsilon\right) .\right.
\end{aligned}
$$

Now one can use Theorem 4.5.8, Lemma 4.4.2 and the elementary properties of the Hopf invariant for tracks in $[9,3.6]$ to check that any track $Q$ from

$$
\left(\Sigma^{n+m}\left(\Omega^{n} X \wedge \sigma^{*}\right),(\cdot)_{n+m}^{\operatorname{sign} \sigma} \wedge \Omega^{n} X \wedge \Omega^{m} Y\right)\left(\left(\Sigma^{n+m-1} \bar{f} \# \bar{g}\right) \vee\left(\Sigma^{n+m-1} \bar{f} \# \bar{g}\right)\right)\left(\Sigma^{n+m-1} \varepsilon\right)
$$

to

$$
\Sigma^{n+m-1}\left(\bar{f} \#\left(\left(\Sigma \sigma^{*},(\cdot)^{\operatorname{sign} \sigma} \wedge \Omega^{m} Y\right)(\bar{g} \vee \bar{g}) \varepsilon\right)\right)
$$

with

$$
\overline{\operatorname{Hopf}(Q)}=-\bar{\sigma} \tau_{\otimes}\left(1 \otimes \tau_{\otimes} \otimes 1\right)\left(H(f) \otimes\left(-\sigma^{*} g+(\operatorname{sign} \sigma)^{*} g \mid \sigma^{*} g\right)_{H}\right),
$$


satisfies

$$
\begin{aligned}
\mathbb{H}_{n, \bar{f}, m,\left(\Sigma \sigma^{*},(\cdot)^{\text {sign } \sigma} \wedge \Omega^{m} Y\right)(\bar{g} \vee \bar{g}) \varepsilon}^{\#} \square Q= & \left(\Sigma^{n+m}\left(\Omega^{n} X \wedge \sigma^{*}\right),\right. \\
& \left.(\cdot)_{n+m}^{\operatorname{sign} \sigma} \wedge \Omega^{n} X \wedge \Omega^{m} Y\right)\left(\mathbb{H}_{n, \bar{f}, m, \bar{g}}^{\#} \vee \mathbb{H}_{n, \bar{f}, m, \bar{g}}^{\#}\right)\left(\Sigma^{n+m-1} \varepsilon\right) .
\end{aligned}
$$

Then given $N:(1,1)\left(\Sigma^{m-1} \varepsilon\right) \Rightarrow 0$ with $\overline{\operatorname{Hopf}}(N)=0$, by using claim $(*)$ in the proof of $[9,4.9]$ and the definition of the bracket $\langle-,-\rangle$ in $[10,4.5]$, the right hand side of equation (1) is

$$
\begin{aligned}
& {\left[(\Sigma \wedge)\left(\Sigma\left(\Omega^{n} X \wedge \sigma^{*}\right),(\cdot)^{\operatorname{sign} \sigma} \wedge \Omega^{n} X \wedge \Omega^{m} Y\right)((\bar{f} \# \bar{g}) \vee(\bar{f} \# \bar{g})) \varepsilon,\right.} \\
& \left(\bar{f}_{e v} \wedge\left(e v\left((\cdot)_{n}^{\operatorname{sign} \sigma} \wedge \Omega^{m} Y\right)\left(\Sigma^{m-1} \bar{g}\right) N\right)\right) \\
& \square\left(\bar{f}_{e v} \wedge\left(e v\left(\hat{\sigma} \wedge \Omega^{m} Y, 0_{(\cdot)_{n}^{\operatorname{sign} \sigma} \wedge \Omega^{m} Y}^{\square}\right)\left(\left(\Sigma^{m-1} \bar{g}\right) \vee\left(\Sigma^{m-1} \bar{g}\right)\right)\left(\Sigma^{m-1} \varepsilon\right)\right)\right) \\
& \square\left(e v\left(\Sigma^{n+m} \wedge\right)\left(\Sigma^{n+m}\left(\Omega^{n} X \wedge \sigma^{*}\right),(\cdot)_{n+m}^{\operatorname{sign} \sigma} \wedge \Omega^{n} X \wedge \Omega^{m} Y\right)\right. \\
& \left.\left.\left(\mathbb{H}_{n, \bar{f}, m, \bar{g}}^{\#} \vee \mathbb{H}_{n, \bar{f}, m, \bar{g}}^{\#}\right)\left(\Sigma^{n+m-1} \varepsilon\right)\right)\right] \\
& =\left[(\Sigma \wedge)\left(\Sigma\left(\Omega^{n} X \wedge \sigma^{*}\right),(\cdot)^{\operatorname{sign} \sigma} \wedge \Omega^{n} X \wedge \Omega^{m} Y\right)((\bar{f} \# \bar{g}) \vee(\bar{f} \# \bar{g})) \varepsilon,\right. \\
& \left(e v\left(\Sigma^{n+m} \wedge\right)\left((\cdot)_{n+m}^{\operatorname{sign} \sigma} \wedge \Omega^{n} X \wedge \Omega^{m} Y\right)\left(\Sigma^{n-1} \bar{f} \wedge \Sigma^{m-1} \bar{g}\right)\left(\Sigma^{n} N\right)\right) \\
& \square\left(e v\left(\Sigma^{n+m} \wedge\right)\left(S^{n} \wedge \hat{\sigma} \wedge \Omega^{n} X \wedge \Omega^{m} Y, 0_{(\cdot)_{n+m}^{\operatorname{sign}} \wedge \Omega^{n} X \wedge \Omega^{m} Y}^{\square}\right)\right. \\
& \left.\left(\left(\Sigma^{n-1} \bar{f} \wedge \Sigma^{m-1} \bar{g}\right) \vee\left(\Sigma^{n-1} \bar{f} \wedge \Sigma^{m-1} \bar{g}\right)\right)\left(\Sigma^{n+m-1} \varepsilon\right)\right) \\
& \square\left(e v\left(\Sigma^{n+m} \wedge\right)\left(\Sigma^{n+m}\left(\Omega^{n} X \wedge \sigma^{*}\right),(\cdot)_{n+m}^{\operatorname{sign} \sigma} \wedge \Omega^{n} X \wedge \Omega^{m} Y\right)\right. \\
& \left.\left.\left(\mathbb{H}_{n, \bar{f}, m, \bar{g}}^{\#} \vee \mathbb{H}_{n, \bar{f}, m, \bar{g}}^{\#}\right)\left(\Sigma^{n+m-1} \varepsilon\right)\right)\right] \\
& =\left[(\Sigma \wedge)\left(\Sigma\left(\Omega^{n} X \wedge \sigma^{*}\right),(\cdot)^{\operatorname{sign} \sigma} \wedge \Omega^{n} X \wedge \Omega^{m} Y\right)((\bar{f} \# \bar{g}) \vee(\bar{f} \# \bar{g})) \varepsilon,\right. \\
& \left(\operatorname{ev}\left(\Sigma^{n+m} \wedge\right)\left((\cdot)_{n+m}^{\operatorname{sign} \sigma} \wedge \Omega^{n} X \wedge \Omega^{m} Y\right)\left(\Sigma^{n-1} \bar{f} \wedge \Sigma^{m-1} \bar{g}\right)\left(\Sigma^{n} N\right)\right) \\
& \square\left(e v\left(\Sigma^{n+m} \wedge\right)\left(S^{n} \wedge \hat{\sigma} \wedge \Omega^{n} X \wedge \Omega^{m} Y, 0_{(\cdot)_{n+m}^{\operatorname{sign} \sigma} \wedge \Omega^{n} X \wedge \Omega^{m} Y}^{\square}\right)\right. \\
& \left.\left.\left(\mathbb{H}_{n, \bar{f}, m, \bar{g}}^{\#} \vee \mathbb{H}_{n, \bar{f}, m, \bar{g}}^{\#}\right)\left(\Sigma^{n+m-1} \varepsilon\right)\right)\right] \\
& =\left[(\Sigma \wedge)\left(\Sigma\left(\Omega^{n} X \wedge \sigma^{*}\right),(\cdot)^{\operatorname{sign} \sigma} \wedge \Omega^{n} X \wedge \Omega^{m} Y\right)((\bar{f} \# \bar{g}) \vee(\bar{f} \# \bar{g})) \varepsilon,\right. \\
& \left(\operatorname{ev}\left(\Sigma^{n+m} \wedge\right)\left((\cdot)_{n+m}^{\operatorname{sign} \sigma} \wedge \Omega^{n} X \wedge \Omega^{m} Y\right)\left(\mathbb{H}_{n, \bar{f}, m, \bar{g}}^{\#}\right)\left(\Sigma^{n} N\right)\right) \\
& \square\left(e v\left(\Sigma^{n+m} \wedge\right)\left(S^{n} \wedge \hat{\sigma} \wedge \Omega^{n} X \wedge \Omega^{m} Y, 0_{(\cdot)_{n+m}^{\operatorname{sign} \sigma} \wedge \Omega^{n} X \wedge \Omega^{m} Y}^{\square}\right)\right. \\
& \left.\left.\left(\left(\Sigma^{n+m-1}(\bar{f} \# \bar{g})\right) \vee\left(\Sigma^{n+m-1}(\bar{f} \# \bar{g})\right)\right)\left(\sum^{n+m-1} \varepsilon\right)\right)\right] \\
& =\left[(\Sigma \wedge)\left(\Sigma\left(\Omega^{n} X \wedge \sigma^{*}\right),(\cdot)^{\operatorname{sign} \sigma} \wedge \Omega^{n} X \wedge \Omega^{m} Y\right)((\bar{f} \# \bar{g}) \vee(\bar{f} \# \bar{g})) \varepsilon,\right. \\
& \left(\operatorname{ev}\left(\Sigma^{n+m} \wedge\right)\left((\cdot)_{n+m}^{\operatorname{sign} \sigma} \wedge \Omega^{n} X \wedge \Omega^{m} Y\right)\left(\Sigma^{n+m-1}(\bar{f} \# \bar{g})\right)\left(\Sigma^{n} N\right)\right) \\
& \square\left(e v\left(\Sigma^{n+m} \wedge\right)\left(S^{n} \wedge \hat{\sigma} \wedge \Omega^{n} X \wedge \Omega^{m} Y, 0_{(\cdot)_{n+m}^{\operatorname{sign} \sigma} \wedge \Omega^{n} X \wedge \Omega^{m} Y}^{\square}\right)\right. \\
& \left.\left.\left(\left(\Sigma^{n+m-1}(\bar{f} \# \bar{g})\right) \vee\left(\Sigma^{n+m-1}(\bar{f} \# \bar{g})\right)\right)\left(\Sigma^{n+m-1} \varepsilon\right)\right)\right] \\
& =\left\langle f \wedge g, S^{n} \wedge \hat{\sigma}\right\rangle
\end{aligned}
$$

Here we essentially use the golden rule, concretely for the fourth equation.

\subsection{The Smash Product in Dimension 0}

The first lemma in this section implies that the smash product operation in Definition 4.3.1 is well defined when dimension 0 is involved and Theorems 3.2.1, 3.2.2, 3.2.3, and 3.3.10 are satisfied also in this case.

Recall that $\mathbf{q p m}$ is a track category. Therefore the morphism set $\operatorname{Hom}_{\mathbf{q p m}}(C, D)$ in $\mathbf{q p m}$ is indeed a groupoid. This groupoid is pointed by the zero morphism. If $G_{\square}$ 
is a sign group acting on $C$ and $D$ we can consider the full pointed subgroupoid of $G_{\square}$-equivariant morphisms

$$
\operatorname{Hom}_{G_{\square}}(C, D) \subset \operatorname{Hom}_{\mathbf{q p m}}(C, D) .
$$

If $G_{\square}$ is the trivial sign group then this inclusion is always an equality.

A pointed groupoid $\mathbf{G}$ gives rise to a stable quadratic module $\operatorname{Ad}_{3} \operatorname{Ad}_{2} \operatorname{Ad}_{1} \mathbf{G}$, compare Remark 3.1.3. The low-dimensional group of this stable quadratic module is the free group of nilpotency class 2 on the pointed set of objects, therefore if we define $H$ as in Eq. 10 we obtain a quadratic pair module $\tilde{\mathbf{G}}$ which corresponds to $\operatorname{Ad}_{3} \operatorname{Ad}_{2} \operatorname{Ad}_{1} \mathbf{G}$ by the forgetful functor in Remark 2.1.3. Compare [10,1.15].

Lemma 4.8.1 Let $C, D$ be quadratic pair modules endowed with an action of the sign group $G_{\square}$, let $\mathbf{G}$ be a groupoid, and let

$$
\varphi: \mathbf{G} \longrightarrow \operatorname{Hom}_{G_{\square}}(C, D)
$$

be a pointed groupoid morphism. Suppose that $C$ is 0-good. Then there is a welldefined quadratic pair module morphism

$$
\tilde{\varphi}: \tilde{\mathbf{G}} \odot C \longrightarrow D
$$

given by

$$
\tilde{\varphi}_{i}(g \odot x)=\varphi(g)(x),
$$

where $g$ is an object in $\mathbf{G}$ and $x \in C_{i}$ for some $i \in\{0,1\}$, or $g$ is a morphism in $\mathbf{G}, x \in C_{0}$ and $i=1$; and $b y$

$$
\tilde{\varphi}_{e e}\left(\left(g \mid g^{\prime}\right)_{H} \otimes\left(x \mid x^{\prime}\right)_{H}\right)=\left(\varphi(g)(x) \mid \varphi\left(g^{\prime}\right)\left(x^{\prime}\right)\right)_{H},
$$

for $g, g^{\prime}$ objects in $\mathbf{G}$ and $x, x^{\prime} \in C_{0}$. This morphism is $G_{\square}$-equivariant.

The proof of the lemma is technical but straightforward. The reader can also check that the construction is natural in $\mathbf{G}, G_{\square}, C$ and $D$.

Finally we show how Lemma 2.5.3 can be used to obtain the tracks in qpm induced by the additive secondary homotopy groups from the smash product operation. For this we notice that there is a unique morphism in $\mathbf{q p m}$ from the interval quadratic pair module $\mathbb{I}$ in Section 2.5 to $\Pi_{0, *}$ of the interval $I_{+}$,

$$
v: \mathbb{I} \longrightarrow \Pi_{0, *} I_{+},
$$

sending $i_{k} \in \mathbb{I}_{0}, k=0,1$, to the map $i_{k}: S^{0} \rightarrow I_{+} \in \Pi_{0,0} I_{+}$corresponding to the inclusion of $k \in I=[0,1]$.

Lemma 4.8.2 Given a track $F: f \Rightarrow g$ between maps $f, g: X \rightarrow Y$ represented by a homotopy $F: I_{+} \wedge X \rightarrow Y$ the composite

$$
\mathbb{I} \odot \Pi_{n, *} X \stackrel{v \odot 1}{\longrightarrow} \Pi_{0, *} I_{+} \odot \Pi_{n, *} X \stackrel{\wedge}{\longrightarrow} \Pi_{n, *}\left(I_{+} \wedge X\right) \stackrel{\Pi_{n, *} F}{\longrightarrow} \Pi_{n, *} Y
$$

corresponds by Lemma 2.5.3 to the track $\Pi_{n, *} F: \Pi_{n, *} f \Rightarrow \Pi_{n, *}$ in $\mathbf{q p m ,} n \geq 0$.

This lemma follows easily from the definition of the smash product operation for secondary homotopy groups.

空 Springer 


\section{Appendix A: Monoidal Structures for Graded Quadratic Pair Modules and Symmetric Sequences}

Let $M$ be any additive monoid. The tensor product of two $M$-graded quadratic pair modules $D, E$ is defined as usually by

$$
(D \odot E)_{n}=\bigvee_{p+q=n} D_{p} \odot E_{q},
$$

where the symbol $\bigvee$ denotes the coproduct. However, since the tensor product of quadratic pair modules does not preserve coproducts, this does not define a monoidal structure on $\mathbf{q p \mathbf { m } ^ { M }}{ }^{M}$. For this we need to restrict to a subcategory of quadratic pair modules where the tensor product preserves coproducts. Below we check that the full subcategory of 0-good quadratic pair modules, already introduced in Definition 2.1.2, is suitable.

The class of good square groups is closed under tensor products and coproducts, see [7] Definition 2 and Section 5.6. The class of 0-good quadratic pair modules is therefore closed under tensor products and coproducts as well. We show in Corollary A. 6 below that the tensor product of 0 -good quadratic pair modules is well-behaved with respect to coproducts. As a consequence we obtain the following result.

Proposition A.1 The category $\mathbf{q p m}_{0}^{M}$ of M-graded 0-good quadratic pair modules is a monoidal category.

The tensor product of symmetric sequences $X, Y$ in $\mathbf{q p m}_{0}^{\text {Sym }_{\square}}$ is similarly well defined by the formula

$$
(X \odot Y)_{n}=\bigvee_{p+q=n}\left(X_{p} \odot Y_{q}\right) \odot_{A\left(\operatorname{Sym}_{\square}(p) \tilde{x} \operatorname{Sym}_{\square}(q)\right)} A\left(\operatorname{Sym}_{\square}(n)\right) .
$$

Here, as usually, if $M$ and $N$ are a right and a left module over a quadratic pair algebra $R$, respectively, then $M \odot_{R} N$ denotes the coequalizer of the two multiplications $M \odot R \odot N \rightrightarrows M \odot N$. The left $A\left(\operatorname{Sym}_{\square}(p) \tilde{x} \operatorname{Sym}_{\square}(q)\right)$-module structure of $A\left(\operatorname{Sym}_{\square}(n)\right)$ is given by the sign group morphism in Proposition 3.3.4. Moreover, we use the characterization of sign group actions given by Lemma 3.3.8 and the fact that $A$ is strict monoidal, see Proposition 3.3.7.

Now we state the technical results of this appendix. For any abelian group $B$ we define following [7] the square group $B^{\otimes}$ as $B_{e}^{\otimes}=B, B_{e e}^{\otimes}=B \oplus B, P=(1,1)$ and $H=\left(\begin{array}{l}1 \\ 1\end{array}\right)$. Clearly $(-)^{\otimes}$ defines a functor from abelian groups to square groups.

Lemma A.2 Given square groups $L, M, N$ there is a natural push-out diagram

$\left(\text { Coker } P_{L} \otimes \text { Coker } P_{M} \otimes \operatorname{Coker} P_{L} \otimes \operatorname{Coker} P_{N}\right)^{\otimes C} \longrightarrow(L \odot M) \vee(L \odot N)$

$$
\cong \downarrow
$$

$\left(\text { Coker } P_{L} \otimes \operatorname{Coker} P_{L} \otimes \operatorname{Coker} P_{M} \otimes \operatorname{Coker} P_{N}\right)^{\otimes}$

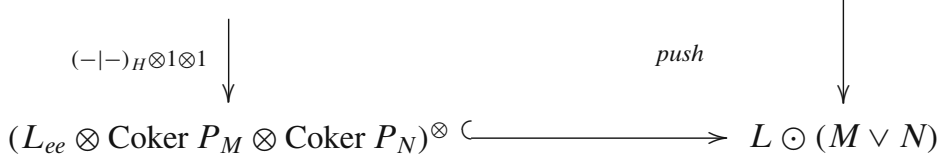


Proof The horizontal arrows are the monomorphisms in the exact sequences of square groups in [7, Proposition 5 and 5.6]. The compatibility of both sequences proves that the square of the statement is indeed a push-out.

Corollary A.3 If $X$ is a good square group then the functor $X \odot-$ preserves coproducts.

Proof By Lemma A.2 the functor $X \odot-$ preserves finite coproducts. Since the tensor product $\odot$ of square groups preserves always filtered colimits, see [7, Proposition 4], then $X \odot-$ preserves indeed arbitrary coproducts.

Lemma A.4 If $\partial: C_{(1)} \rightarrow C_{(0)}$ is a quadratic pair module such that $C_{(0)} \odot$ - preserves coproducts then for any two quadratic pair module morphisms $f_{i}: M_{i} \rightarrow N_{i}, i=1,2$, the natural morphism

$$
\Phi\left(\left(\partial \odot f_{1}\right) \vee\left(\partial \odot f_{2}\right)\right) \longrightarrow \Phi\left(\partial \odot\left(f_{1} \vee f_{2}\right)\right)
$$

in $\mathbf{q p m}$ is an epimorphism. Moreover it is an isomorphism provided $f_{i}$ are identity morphisms $i=1,2$.

Proof Since $C_{(0)} \odot$ - preserves coproducts the morphism is an isomorphism on the (0)-level. The reflection functor $\Phi$ from pairs of square groups to quadratic pair modules is a left adjoint, so it preserves colimits. In particular by Lemma A.2 the first part of this lemma holds provided the natural morphism

$$
\begin{gathered}
A=\Phi\left(\left(\text { Coker } P_{C} \otimes \operatorname{Coker} P_{C} \otimes \operatorname{Coker} P_{M_{1}} \otimes \operatorname{Coker} P_{M_{2}}\right)^{\otimes} \rightarrow C_{(0)} \odot\left(N_{1} \vee N_{2}\right)\right) \\
\downarrow \\
B=\Phi\left(\left(C_{e e} \otimes \operatorname{Coker} P_{M_{1}} \otimes \operatorname{Coker} P_{M_{2}}\right)^{\otimes} \rightarrow C_{(0)} \odot\left(N_{1} \vee N_{2}\right)\right)
\end{gathered}
$$

induced by $(-\mid-)_{H}: \otimes^{2}$ Coker $P_{C} \rightarrow C_{e e}$ is surjective on the 1-level. On this level $A$ and $B$ are quotient groups, $A_{1}=\tilde{A}_{1} / \sim, B_{1}=\tilde{B}_{1} / \sim$, of the abelian groups

$$
\begin{aligned}
& \tilde{A}_{1}=\text { Coker } P_{C} \otimes \text { Coker } P_{C} \otimes \text { Coker } P_{M_{1}} \otimes \text { Coker } P_{M_{2}} \oplus D, \\
& \tilde{B}_{1}=C_{e e} \otimes \text { Coker } P_{M_{1}} \otimes \text { Coker } P_{M_{2}} \oplus D .
\end{aligned}
$$

and the morphism $A \rightarrow B$ is induced by $(-\mid-)_{H}: \otimes^{2}$ Coker $P_{C} \rightarrow C_{e e}$ and the identity on $D$. Here $D$ is the abelian group

$$
D=C_{e e} \otimes\left(\left(N_{1}\right)_{e e} \oplus\left(N_{2}\right)_{e e} \oplus \operatorname{Coker} P_{N_{1}} \otimes \operatorname{Coker} P_{N_{2}} \oplus \text { Coker } P_{N_{2}} \otimes \operatorname{Coker} P_{N_{1}}\right) .
$$

The relations $\sim$ in Section 2.4 and the definition of $(-)^{\otimes}$ show that $A_{1}$ and $B_{1}$ are generated by image of $D$, and hence (a) is surjective on the 1-level.

Now let $f_{i}=1: M_{i}=N_{i}, i=1,2$. In order to check the second part of the statement it is enough to show that the morphism

$$
\begin{gathered}
A=\Phi\left(\left(\text { Coker } P_{C} \otimes \text { Coker } P_{C} \otimes \operatorname{Coker} P_{N_{1}} \otimes \operatorname{Coker} P_{N_{2}}\right)^{\otimes} \rightarrow C_{(0)} \odot\left(N_{1} \vee N_{2}\right)\right) \\
\downarrow \\
\Phi\left(\left(C_{(1)} \odot N_{1}\right) \vee\left(C_{(1)} \odot N_{2}\right) \rightarrow C_{(0)} \odot\left(N_{1} \vee N_{2}\right)\right)
\end{gathered}
$$


given by the upper vertical arrow in Lemma A.2 factors through (a). Both (a) and (b) are the identity on the (0)-level, so it is enough to look at the (1)-level. The desired factorization is induced by the unique square group morphism

$$
\begin{gathered}
\left(C_{e e} \otimes \operatorname{Coker} P_{N_{1}} \otimes \operatorname{Coker} P_{N_{2}}\right)^{\otimes} \\
\downarrow \\
\downarrow\left(\left(C_{(0)} \odot N_{1}\right) \vee\left(C_{(0)} \odot N_{2}\right) \rightarrow C_{(0)} \odot\left(N_{1} \vee N_{2}\right)\right)_{1}
\end{gathered}
$$

which coincides on the ee-level with the square group morphism

$$
\left(C_{e e} \otimes \text { Coker } P_{N_{1}} \otimes \text { Coker } P_{N_{2}}\right)^{\otimes} \longrightarrow C_{(0)} \odot\left(N_{1} \vee N_{2}\right),
$$

given by the lower horizontal arrow in Lemma A.2.

Lemma A.5 Let $C$ be a quadratic pair module such that $C_{(0)} \odot-$ preserves coproducts. Then $\mathrm{C} \odot-$ also preserves coproducts.

Proof Let us first check that $C \odot-$ preserves the coproduct of two quadratic pair modules $D, E$. The functor $\Phi$ preserves colimits since it is a left adjoint. The tensor product of quadratic pair modules is defined by $\Phi$ and the push-out construction in Eq. 12 in the category SG of square groups, which is also a push-out in the category Pair(SG) of pairs of square groups. Therefore it is enough to check that the natural morphism

$$
\begin{gathered}
\Phi\left(\left(C_{(i)} \odot D_{(j)}\right) \vee\left(C_{(i)} \odot E_{(j)}\right) \rightarrow C_{(0)} \odot\left(D_{(0)} \vee E_{(0)}\right)\right) \\
\downarrow \\
\downarrow\left(C_{(i)} \odot\left(D_{(j)} \vee E_{(j)}\right) \stackrel{\rightarrow}{\rightarrow} C_{(0)} \odot\left(D_{(0)} \vee E_{(0)}\right)\right)
\end{gathered}
$$

is an isomorphism if $i=0$ or $j=0$, and an epimorphism if $i=j=1$, and this follows from the previous lemma.

Since the coproduct of two objects is preserved by $C \odot-$ then all finite coproducts are preserved. Moreover, by [7, Proposition 4] the tensor product of square groups preserves filtered colimits. Since $\Phi$ preserves colimits then the tensor product of quadratic pair modules also preserves filtered colimits, hence $C \odot-$ indeed preserves arbitrary coproducts.

Corollary A.6 If $C$ is a 0-good quadratic pair module then $C \odot-$ preserves coproducts.

\section{References}

1. Baues, H.-J.: Commutator calculus and group of homotopy classes. In: Lecture Notes, no. 50. Cambridge University Press, Cambridge (1981)

2. Baues, H.-J.: Combinatorial Homotopy and 4-Dimensional Complexes. Walter de Gruyter, Berlin (1991)

3. Baues, H.-J.: The algebra of secondary cohomology operations. In: Progress in Mathematics, vol. 247. Birkhäuser, Cambridge, MA (2006)

4. Barcus, W.D., Barratt, M.G.: On the homotopy classification of the extensions of a fixed map. Trans. Amer. Math. Soc. 88, 57-74 (1958)

5. Baues, H.-J., Jibladze, M.: Suspension and loop objects and representability of track. Georgian Math. J. 8(4), 683-696 (2001) 
6. Barratt, M.G., Jones, J.D.S., Mahowald, M.E.: The Kervaire invariant problem. In: Proceedings of the Northwestern Homotopy Theory Conference, Evanston, IL, 1982. Contemporary Mathematics, vol. 19, pp. 9-22. American Mathematical Society, Providence, RI (1983)

7. Baues, H.-J., Jibladze, M., Pirashvili, T.: Quadratic algebra of square groups. Preprint of the Max-Planck-Institut für Mathematik MPIM2006-9, http://arxiv.org/abs/math.CT/0601777 (2005)

8. Baues, H.-J., Muro, F.: Secondary algebras associated to ring spectra. Preprint of the MaxPlanck-Institut für Mathematik MPIM2006-137, http://arxiv.org/abs/math.AT/0610523 (2006)

9. Baues, H.-J., Muro, F.: Secondary homotopy groups. Preprint of the Max-Planck-Institut für Mathematik MPIM2006-36. http://arxiv.org/abs/math.AT/0604029. Forum Mathematicum (2006)

10. Baues, H.-J., Muro, F.: The symmetric action on secondary homotopy groups. Preprint of the Max-Planck-Institut für Mathematik MPIM2006-37. http://arxiv.org/abs/math.AT/0604030 (2006)

11. Baez, J.C., Neuchl, M.: Higher-dimensional algebra. I. Braided monoidal 2-categories. Adv. Math. 121(2), 196-244 (1996)

12. Borceux, F.: Handbook of categorical algebra 1. In: Encyclopedia of Mathematics and Its Applications, no. 50. Cambridge University Press, Cambridge (1994)

13. Baues, H.-J., Pirashvili, T.: Quadratic endofunctors of the category of groups. Adv. Math. 141, 167-206 (1999)

14. Bröcker, T., Dieck, tom T.: Representations of compact Lie groups. In: Graduate Texts in Mathematics, vol. 98. Springer, New York (1985)

15. Conduché, D.: Modules croisés généralisés de longueur 2. J. Pure Appl. Algebra 34(2-3), 155-178 (1984)

16. Crans, S.E.: Generalized centers of braided and sylleptic monoidal 2-categories. Adv. Math. 136(2), 183-223 (1998)

17. Ellis, G.: Crossed squares and combinatorial homotopy. Math. Z. 214(1), 93-110 (1993)

18. Hardie, K.A., Mahowald, M.E.: On cup-one and Toda bracket. Quaestiones Math. 16(2), 193207 (1993)

19. Hovey, M., Shipley, B., Smith, J.: Symmetric spectra. J. Amer. Math. Soc. 13(1), 149-208 (2000)

20. Marcum, H.J.: Obstructions for a map to be cyclic. In: Algebraic Topology, Oaxtepec, 1991. Contemporary Mathematics, vol. 146, pp. 277-295. American Mathematical Society, Providence, RI (1993)

21. Rutter, J.W.: A homotopy classification of maps into an induced fibre space. Topology 6, 379-403 (1967)

22. Toda, H.: Composition methods in homotopy groups of spheres. In: Annals of Mathematics Studies, no. 49. Princeton University Press, Princeton, NJ (1962) 